\title{
Modelagem epidêmica por meio de técnicas da mecânica quântica de muitos corpos
}

Dissertação apresentada à Faculdade de Filosofia, Ciências e Letras de Ribeirão Preto da Universidade de São Paulo como parte das exigências para a obtenção do título de Mestre em Ciências.

Área de Concentração:

Física Aplicada à Medicina e Biologia.

Orientador:

Alexandre Souto Martinez.

Versão original

Disponível na FFCLRP-USP

Ribeirão Preto 
Autorizo a reprodução e divulgação total ou parcial deste trabalho, por qualquer meio convencional ou eletrônico, para fins de estudo e pesquisa, desde que citada a fonte.

\section{FICHA CATALOGRÁFICA}

Gomes, Naomy Duarte

Modelagem epidêmica por meio de técnicas da mecânica quântica de muitos corpos / Naomy Duarte Gomes; orientador: Alexandre Souto Martinez. - Ribeirão Preto, 2019.

$44 \mathrm{f}$ : : il.

Dissertação (Mestrado) - - Faculdade de Filosofia, Ciências e Letras de Ribeirão Preto, Universidade de São Paulo, 2019.

Inclui Bibliografia.

1. Física estatística 2. Modelos epidêmicos 3. Processos estocásticos 4. Estados coerentes 
Nome: Gomes, Naomy Duarte

Título: Modelagem epidêmica por meio de técnicas da mecânica quântica de muitos corpos

Dissertação apresentada á Faculdade de Filosofia, Ciências e Letras de Ribeirão Preto da Universidade de São Paulo como parte das exigências para a obtenção do título de Mestre em Ciências.

Aprovado em:

\section{Banca Examinadora}

$\operatorname{Prof}(\mathrm{a}) \cdot \operatorname{Dr}(\mathrm{a})$.

Julgamento:

$\operatorname{Prof}(\mathrm{a}) . \operatorname{Dr}(\mathrm{a})$.

Julgamento:

$\operatorname{Prof}(\mathrm{a}) . \operatorname{Dr}(\mathrm{a})$.

Julgamento:
Instituição:

Assinatura:

Instituição:

Assinatura:

Instituição:

Assinatura: 
Para elas, sempre: Shirley, Nathaly e a eterna Dona Lia. 
Não sou nada, nunca serei nada, não posso querer ser nada. À parte isso, tenho em mim toda a curiosidade do mundo. 


\section{Resumo}

GOMES, N.D. Modelagem epidêmica por meio de técnicas da mecânica quântica de muitos corpos. 2019. 44 f. Dissertação (Mestrado - Programa de Pós-Graduação em Física Aplicada à Medicina e Biologia) - Faculdade de Filosofia, Ciências e Letras de Ribeirão Preto, Universidade de São Paulo, Ribeirão Preto, 2019.

Modelos matemáticos de transmissão de doenças tiveram início com o trabalho de McKendrick e Kermack em 1927. Desde então, inúmeros modelos foram formulados para analisar a dinâmica da transmissão de doenças, parâmetros epidemiológicos tais como taxas de cura e de transmissão e possíveis efeitos de uma rede de contatos e do ambiente. Estes últimos são variáveis que introduzem o caráter estocástico das epidemias e sua consideração torna a modelagem mais próxima da realidade. As análises de processos epidêmicos e estimativas de parâmetros são de grande importância para a sociedade, uma vez que podem ser utilizadas no estudo de políticas de intervenção para reduzir ou extinguir a transmissão da doença. No entanto, modelos epidêmicos são, em sua maioria, determinísticos e simplificados. De modo a serem mais próximos da realidade, é importante que os modelos incluam efeitos que levam em conta a heterogeneidade da população e possíveis flutuações estocásticas. Neste trabalho, para o modelo epidêmico SIS, consideramos um modelo estocástico que leva em conta a rede de contatos adjacente e investigamos o papel das flutuações para pequenas populações, definindo um sistema de equações fechado no qual tem-se a evolução temporal tanto da densidade de infectados quanto da flutuação. No limite no qual a população considerada é muito grande, as flutuações são irrelevantes e podem ser tomadas como constantes. Desta forma, introduzimos os estados coerentes, utilizando a transformação de Holstein-Primakoff, e usamos as propriedades destes estados a fim de construir equações para estimar parâmetros epidemiológicos.

Palavras-chave: 1. Física estatística 2. Modelos epidêmicos 3. Processos estocásticos 4. Estados coerentes 


\section{Abstract}

GOMES, N.D. Modeling epidemics via quantum many-body techniques. 2019. 44 f. Dissertation (M.Sc. - Postgraduate program in Physics Applied to Medicine and Biology) - Faculty of Philosophy, Sciences and Letters, University of São Paulo, Ribeirão Preto, 2019.

Mathematical models of disease transmission in populations began with the work of McKendrick and Kermack in 1927 and since then numerous models have been formulated to analyze, among others, the dynamics of the disease spreading process, epidemiological parameters such as the rates of cure and transmission and the possible effects of the underlying network and the environment. The latter are variables that introduce the stochastic nature of epidemics, and their consideration makes the modeling closer to reality. The analysis of epidemic processes and estimates of epidemiological parameters are of great importance to society since they can be used to study effective intervention policies to stop or reduce the spreading of the disease. However, epidemic models are, in most cases, deterministic and simplistic. To be closer to reality, it is essential to include effects that account for the heterogeneity of the population and possible stochastic fluctuations. In this work, for the SIS epidemic model, we consider a stochastic model that takes into account the adjacent network, and we investigate the role of fluctuations for small populations, defining a closed system of equations for which we have the evolution of both the density of infected and the fluctuation itself. In the limit where the considered population is high, fluctuations are irrelevant and can be taken as constant. With this consideration, we introduce coherent states through the Holstein-Primakoff transformation and use the properties of these states to construct equations that allow for the estimation of epidemiological parameters.

Key-words: 1. Statistical Physics 2. Epidemic models 3. Stochastic processes 4. Coherent States 


\section{Lista de Figuras}

2.1 Representação dos microorganismos causadores de doenças e das diversas vias de transmissão. . . . . . . . . . . . . . . . . . . . 7

2.2 Doenças endêmicas, epidemias e pandemia. . . . . . . . . . . . . . 9

3.1 a) Representação esquemática do modelo compartimental contendo indivíduos que podem ser classificados como suscetíveis (S), expostos (E), infectados (I) e recuperados (R). A dinâmica do processo se dá de tal forma que indivíduos suscetíveis podem se tornar expostos através do contato com infectados. Uma vez adquirida a doença, tornam-se infectados. Em alguns casos possíveis (representados pelas linhas pontilhadas), os indivíduos podem se recuperar da doença, tornando-se resistentes ou imunes e entrando na classe de recuperados. Indivíduos recuperados podem, no entanto, tornar-se suscetíveis novamente, como ocorre no caso de doenças às quais se adquire imunidade apenas por um período (gripe, por exemplo). . . . . . . . . . 13

3.2 a) Representação esquemática do modelo compartimental, no qual os indivíduos são divididos em várias subclasses. b) Equações diferenciais derivadas do modelo compartimental. Figura retirada do trabalho de S. Del Valle et al, Mathematical Biosciences 195 (2005). . . . . . . 14

3.3 Representação esquemática de um grafo com diferentes graus de conectividade, no qual alguns indivíduos em vértices mais distantes são menos conectados do que aqueles no centro. Informações sobre a estrutura do grafo podem ser retiradas de censos populacionais, por exemplo. . . . . . . . . . . . . . . . . . . 15 
4.1 Modelo SIS compartimental, onde ocorre a troca entre compartimentos com indivíduos suscetíveis (azul) e infectados (vermelho). . . . . 19

4.2 Indivíduos infectados e suscetíveis identificados por um indíce inteiro e cuja interação é representada pelas linhas conectando cada um deles. 22

4.3 Todas as 8 configurações possíveis para $N=3$. Em vermelho, indivíduos infectados; em azul, suscetíveis. . . . . . . . . . . . . . 23

4.4 Representação de um grafo completo, com a matriz de adjacência dada por $A_{j k}=1-\delta_{j k}$, no qual todos os indivíduos nos vértices de 1 a 8 estão conectados entre si. Imagem retirada da Wikipedia. . . . . . 28

4.5 Evolução da densidade de infectados como função do tempo (linhas cheias). Para valores de $\gamma / \alpha=0.1,0.2,0.3$, portanto com alta taxa de infecção e baixa taxa de cura, o número de infectados aumenta muito e rapidamente, atingindo os valores $\rho_{e q}$ previstos pela equação compartimental (linhas tracejadas com cores correspondentes aos respectivos valores de $\gamma / \alpha$ ), sendo a variância muito próxima de zero. Conforme a taxa de cura aumenta, o número de infectados diminui para um valor abaixo do valor de equilíbrio. Simulações de Monte Carlo realizadas com $10^{6}$ amostras e para $N=50$. . . . . . . . 32

4.6 Evolução temporal do número de infectados para diferentes valores de $N$ : a) $\gamma / \alpha=0.1$, b) $\gamma / \alpha=0.3$, c) $\gamma / \alpha=0.5$ e d) $\gamma / \alpha=0.9$. Em todos os casos, podemos observar que quanto menores os valores de $N$, mais as flutuações agem diminuindo a densidade de infectados no equilíbrio. No começo da epidemia, no entanto, tem-se o efeito contrário. Nota-se também que os efeitos de flutuação são menores para valores de $\gamma / \alpha$ baixos em relação ao observado para valores de $\gamma / \alpha$ altos, com uma diferença máxima aparente para $\gamma / \alpha=0.5 \mathrm{e}$ $N=10$. Simulações de Monte Carlo realizadas com $10^{6}$ amostras. . . 33

4.7 Evolução temporal da derivada da variância para diferentes valores de $\gamma / \alpha$ com comportamento esperado no equilíbrio ( $\sigma^{2}$ constante), usando os dados simulados. As linhas cheias representam os valores fornecidos usando a Eq. (4.35). Simulações de Monte Carlo realizadas com $10^{6}$ amostras e para $N=50$. . . . . . . . . . . . . . 35 
$4.8 \Delta_{3}(t)$ como função de $\langle\rho\rangle \sigma^{2}$ para valores de $\gamma / \alpha$ variáveis e $N=50$. No limite $N \gg 1$, as flutuações são bem representadas por flutuações Gaussianas, com $\Delta_{3}(t)=3\langle\rho\rangle \sigma^{2}$, apenas para valores de $\gamma / \alpha$ pequenos. Nota-se que, para $\gamma / \alpha=0.8$ e $0.9, \Delta_{3}(t)$ deixa de ser uma função de $\langle\rho\rangle \sigma^{2}$, de modo que, nestes casos, a aproximação Gaussiana falha. Simulação de Monte Carlo realizada com $10^{6}$ amostras. . . . . 36

$4.9 \Delta_{3}(t)$ como função de $\langle\rho\rangle \sigma^{2}$ para valores de $N$ variáveis e com $\gamma / \alpha=$ 0.1. Pode-se notar a dependência da aproximação Gaussiana com o valor de $N$. Para $N=30,40$ e 50, obtém-se a reta esperada $\Delta_{3}(t)=3\langle\rho\rangle \sigma^{2}$, porém, para $N=10$ e $20, \Delta_{3}(t)$ deixa de ser uma função de $\langle\rho\rangle \sigma^{2}$, e a aproximação Gaussiana falha. . . . . . . . . . . . 37

4.10 Derivada segunda em relação ao tempo da densidade de infectados. Simulação de Monte Carlo realizada utilizando-se a Eq. (4.39), com $10^{6}$ amostras e para $N=50$, com valores de $\gamma / \alpha$ variáveis. . . . . . 38

4.11 Evolução temporal da derivada da variância para as Eqs. (4.35) e (4.37). a) $\gamma / \alpha=0.1$, b) $\gamma / \alpha=0.3$, c) $\gamma / \alpha=0.5$ e d) $\gamma / \alpha=$ 0.9. Observa-se um comportamento semelhante de $d \sigma^{2} / d t$ tanto para o caso com flutuações Gaussianas (Eq. (4.37)) quanto para o caso geral (Eq. (4.35)) sem aproximações. Simulações de Monte Carlo realizadas com $10^{6}$ amostras e para $N=50$. . . . . . . . . . . 39

4.12 Evolução temporal de $D_{\rho \rho}(t) /\langle\rho(t)\rangle$ para valores de $N$ variáveis e $\gamma / \alpha=0.8$ e 0.9 . Para estes valores de $\gamma / \alpha$ e $N=10,20$, a consideração de flutuações Gaussianas falha. Nestes casos, a função de autocorrelação normalizada pode ser usada para substituir o termo $\Delta_{3}$, com $D_{\rho \rho}(t) /\langle\rho(t)\rangle$ apresentando um crescimento exponencial. Simulações de Monte Carlo realizadas com $10^{6}$.

5.1 a) Spins em uma rede quadrada de um material ferromagnético alinham-se no mesmo sentido, com constante de acoplamento $J<0$ e b) spins em um material antiferromagnético alinham-se em sentidos opostos aos dos vizinhos mais próximos, com constante de acopla-

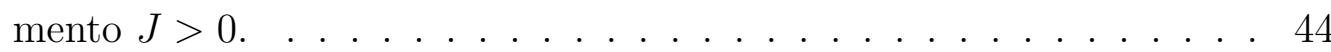


5.2 a) Sistema massa-mola com a posição da massa dada pela linha tracejada em vermelho e b) soluções da Equação de Schrödinger para o oscilador harmônico quântico, no qual o eixo horizontal representa a posição da função de onda, e o eixo vertical, as partes real (em azul) e imaginária (em vermelho) da função de onda. Fonte: Wikipedia. . . 46

5.3 Uma homenagem ao físico Roy Jay Glauber (1925-2018) com seus estados coerentes. . . . . . . . . . . . . . . . 48 


\section{Sumário}

Lista de Figuras $\quad$ xiii

1 Introdução 1

2 Noções e conceitos de epidemiologia 5

2.1 Introdução a doenças comunicáveis . . . . . . . . . . . . . . . . . . 6

2.2 Definições e importância do controle de doenças . . . . . . . . . . . . 8

3 Modelagem matemática de epidemias $\quad 11$

3.1 Modelos compartimentais e determinísticos . . . . . . . . . . . . . 12

3.2 Refinamentos do modelo compartimental . . . . . . . . . . . . . . 14

$\begin{array}{lll}4 & \text { Modelagem baseada em agentes } & 17\end{array}$

4.1 Modelo compartimental . . . . . . . . . . . . . . . . . 18

4.2 Modelo baseado em agentes . . . . . . . . . . . . . . . . . 20

4.3 Espaço vetorial, operadores e matriz de transição . . . . . . . . . . . 21

4.4 Flutuações . . . . . . . . . . . . . . . . . . . . . . . . . 27

4.5 Flutuações Gaussianas . . . . . . . . . . . . . . . . . . . . 35

4.6 Função de correlação . . . . . . . . . . . . . . . . . . . . . . . . . . . 40

4.7 Conclusões do capítulo . . . . . . . . . . . . . . . . . . . . . . . . 41

5 Estados Coerentes $\quad 43$

5.1 Oscilador harmônico quântico . . . . . . . . . . . . . . . . 45

5.2 Estados coerentes: definição e propriedades . . . . . . . . . . . . . . 47

5.3 Aplicação no modelo SIS . . . . . . . . . . . . . . . . . . . . . 50

5.4 Conclusões do capítulo . . . . . . . . . . . . . . . . . . . . . 55 
xviii

6 Conclusão e perspectivas $\quad 57$

Apêndice 1 - Transformação de Holstein-Primakoff 61

Apêndice 2 - Potências do operador $\hat{n} \quad 65$

$\begin{array}{ll}\text { Referências } & 67\end{array}$ 


\section{Introdução}

Doenças infecciosas estão presentes na humanidade desde seus primórdios e foram responsáveis por milhões de mortes globalmente em 2016, que ocorreram principalmente em países subdesenvolvidos e em desenvolvimento [1]. Muitos esforços têm sido feitos para controlar e erradicar essas doenças. Há diversos estudos buscando modelos que ofereçam a melhor adequação à população e ao ambiente considerados [2-4]. Populações muito grandes, como a de grandes metrópoles, e populações muito pequenas, como a de vilarejos, requerem abordagens diferentes, assim como diferentes doenças.

Apesar dos diversos modelos já propostos, um dos problemas em epidemiologia consiste no fato de que é bem difícil simular em laboratório o comportamento das diversas doenças que são transmitidas, diferentemente de sistemas físicos como átomos frios, por exemplo, que podem ser realizados em laboratório e os parâmetros envolvidos podem ser controlados com grande acurácia [5, 6]. Obter dados completos sobre doenças não é uma tarefa fácil e de precisão, o que então requer estudos aprofundados sobre maneiras de se lidar com a falta de informação [7]. Por estes motivos, modelar um problema epidemiológico não permite prever exatamente o comportamento do espalhamento da doença, mas pode tornar possível uma análise mais detalhada de parâmetros que sejam importantes para o seu controle, de modo que estratégias de intervenção possam ser realizadas de forma mais eficaz. Um exemplo bem sucedido é o trabalho de Ronald Ross com a malária [8], onde ele identifica o estágio mais vulnerável do ciclo de vida do parasita e sugere uma intervenção efetiva, que resultou em políticas de controle do mosquito e diminuição do número de casos da doença. 
Devido à dificuldade quantos aos dados empíricos, a importância de se entender como ocorre a infecção e a transmissão de doenças passa a ter um papel central na epidemiologia. Quando o ser humano passa a reconhecer a existência de vírus, bactérias e parasitas, torna-se mais fácil entender por quais motivos uma infecção ocorre e quais hábitos e/ou lugares devem ser evitados para não haver risco de contaminação. De maneira geral, entender e classificar os diversos tipos de formas de transmissão de uma doença e classificar a magnitude das infecções em uma população permite abordar as melhores maneiras de se tratar a doença e evitá-la. Por isto, no capítulo 2 são definidos os conceitos-base das doenças infecciosas e é apresentada a importância das políticas públicas de combate ao surgimento de doenças e de tratamento de infectados.

O capítulo 3 aborda a modelagem matemática da transmissão de doenças, que é uma maneira robusta de fazer previsões com base em dados. São introduzidos os modelos compartimentais que, apesar de terem surgido em 1927, até hojem permacem aplamente utilizados $[9,10]$, devido à sua simplicidade e capacidade de fazer boas previsões para grandes populações. Porém, muitas vezes eles são aliados a refinamentos para que melhor se adequem à realidade da população considerada. Isto pode ser feito com o uso de rede de conexões heterogênea, que podem levar em conta diferentes fatores de suscetibilidade à doença como gênero, separação geográfica e idade. Por isso, são também abordados neste capítulos os modelos compartimentais heterogêneos e os modelos estocásticos, importantes quando se trata de pequenas populações ou quando o interesse está em analisar probabilidades.

No capítulo 4, é introduzido o modelo compartimental base deste trabalho, que contempla doenças às quais um indivíduo não adquire imunidade, o que faz com que ele possa oscilar entre dois estados: suscetível à doença ou infectado. Como forma de refinar o modelo compartimental, é definido um processo epidêmico estocástico no qual são utilizadas técnicas de muitos corpos em física. Este processo tem como resultado a previsão do comportamento da doença para pequenas populações nas quais flutuações são um fator relevante.

Por fim, o capítulo 5 utiliza o modelo compartimental estocástico do capítulo 4, mas considera grandes populações e introduz os estados coerentes na modelagem matemática, minimizando assim as flutuações e buscando obter equações que repre- 
sentem o número médio de infectados e taxas de cura e infecção. 


\section{2}

\section{Noções e conceitos de epidemiologia}

A humanidade presenciou diversos casos de doenças de grandes proporções que alteraram significativamente o curso da história. Entre os anos 1340 e 1350, a peste negra, causada pela bactéria Yersinia pestis, se espalhou pela Europa e Ásia diversas vezes, muito provavelmente devido aos ratos dos navios mercantes, que carregavam consigo a pulga transmissora da doença. Ela resultou em milhões de mortes, dizimando uma alta parcela da população européia. Notavelmente, foi graças à peste negra que Isaac Newton ficou isolado em sua casa e, durante este tempo, inventou o cálculo diferencial, descobriu a lei da gravitação, dentre outras descobertas [11].

Durante os anos 1500, quando da invasão dos espanhóis ao México, a população asteca foi exposta a vírus aos quais não possuía nenhuma imunidade, sendo infectada com doenças como catapora, que contribuíram para a dizimação dos astecas. Quase 500 anos depois, durante os anos 1970, o mundo conhecia os casos de doenças sexualmente transmissíveis (DST), como a síndrome da imunodeficiência adquirida (AIDS, siga em inglês), que foram relatados em diversas partes do mundo, mas que foi reconhecida como uma nova doença em 1981 [12]. Desde então, milhões de pessoas já foram infectadas e mortas devido à AIDS, que hoje em dia, apesar das campanhas de combate, ainda é um grande problema de saúde pública. Mais recentemente, o caso do Ebola, doença adquirida pelo contato com fluidos corporais, chocou o mundo com o grande surto de 2014 na África, que foi declarado pela Organização Mundial de Saúde (OMS) como uma emergência de saúde 
pública internacional. Além de ter causado muitas mortes, o Ebola contribuiu para a deterioração da economia dos países africanos afetados [13].

É notável, conforme os exemplos acima citados, que doenças comunicáveis afetam populações de maneiras distintas, gerando mortes e outros efeitos em larga escala, que atingem os seres humanos de diversas formas: social, economica e tecnologicamente, o que as coloca como questões de grande importância e de preocupação global.

Neste capítulo, é feita uma introdução às doenças comunicáveis, com as definições dos seus meios de transmissão. São definidas também as diferentes classificações do aparecimento de doenças e sua capacidade de propagação. Por fim, é chamada a atenção para a importância do tratamento e da busca pela extinção de doenças.

\subsection{Introdução a doenças comunicáveis}

As doenças comunicáveis são causadas por patógenos, que são microorganismos como vírus, bactérias, parasitas e fungos, sendo transmitidas de pessoa para pessoa ou de animal para pessoa de diversas maneiras, direta ou indiretamente, tais como:

i) contato direto com fluidos corporais de pessoas infectadas, como saliva, sangue, secreções vaginais ou sêmen. Exemplos incluem DST, ebola e influenza;

ii) picadas de insetos ou contato com outros animais que transmitem a doença, cujos exemplos incluem a malária, transmitida pela fêmea infectada do mosquito Anopheles. Pode-se citar também a febre amarela, dengue, febre zica e chikungunha, todas transmitidas pelo Aedes aegypti, e a peste negra, transmitida por pulgas;

iii) disseminação do patógeno por via aérea. Como exemplo pode-se citar a tuberculose, cujas bactérias Mycobacterium tuberculosis são transmitidas por via área com a disseminação de gotículas nas quais elas estejam contidas. A disseminação pode ocorrer por tosse, espirro ou fala do indivíduo contaminado;

iv) alimentos e água contaminados com agentes infecciosos. Exemplos incluem a salmonela, doença gerada por bactérias do gênero Salmonella e que podem ser contraídas através de alimentos de origem aviária mal cozidos, como o ovo. A 
coléra, doença causada pela bactéria Vibrio cholerae, e a giardíase, causada pelo protozoário Giardia lamblia, também são transmitidas pela água e alimentos contaminados por fezes que contenham os patógenos.

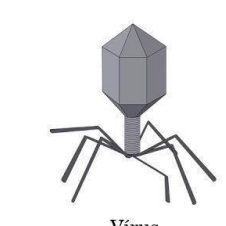

Vírus

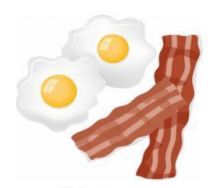

Alimentação

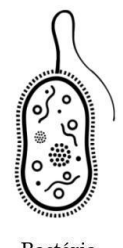

Bactéria
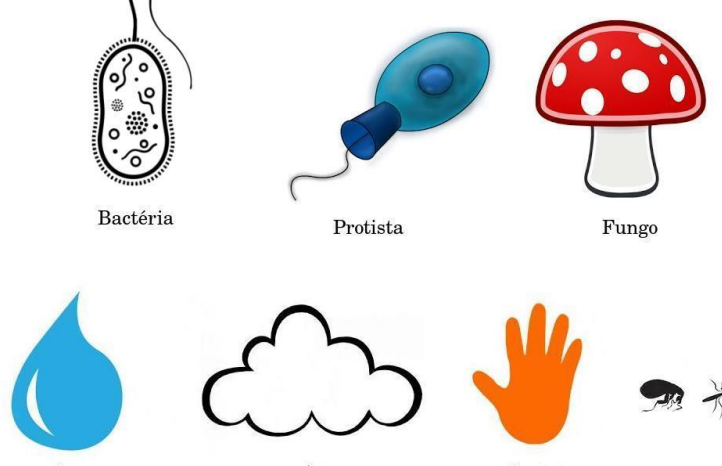

Água

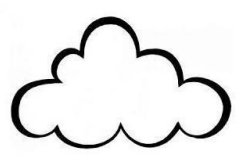

$\mathrm{Ar}$

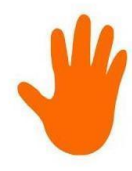

Contato

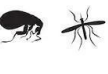

Insetos

Figura 2.1: Representação dos microorganismos causadores de doenças e das diversas vias de transmissão.

O corpo humano, ao entrar em contato com algumas doenças comunicáveis, pode tornar-se resistente ao patógeno associado a elas, produzindo anticorpos específicos para o organismo invasor. Nestes casos, diz-se que o indivíduo adquire imunidade à doença. O princípio de funcionamento da vacina é justamente este, o de expor o sistema imunológico a vírus e bactérias que sejam capazes de estimular o sistema imunológico a produzir anticorpos, porém sem provocar a doença. Por isso a vacinação torna-se tão importante quando se trata de doenças às quais pode-se adquirir imunidade, pois ela age tornando os vacinados imunes. Alguns exemplos de doenças para os quais existem vacinas incluem catapora, varíola, caxumba e sarampo.

Outras doenças como influenza, cuja vacina confere imunidade apenas por um período, e algumas DST (sífilis, gonorréia) podem ser adquiridas diversas vezes ao longo da vida de um indivíduo. Nestes casos, políticas públicas de prevenção, tais como conscientização da população, divulgação de hábitos de higiene adequados e distribuição de preservativos (para o caso das DST), tornam-se muito importantes na tentativa de evitar a contaminação. 


\subsection{Definições e importância do controle de doenças}

O aparecimento de doenças em populações recebe classificações de acordo com a distribuição dos casos na região considerada. Um surto de uma determinada doença ocorre quando há um aumento maior do que o esperado pelas autoridades de uma determinada doença e que esta surge em regiões específicas, como bairros. Um exemplo foi o que ocorreu na cidade de Itajaí - SC em 2015, quando um surto de dengue na região portuária levou ao cancelamento do carnaval da cidade e a políticas públicas de intervenção imediata para evitar uma epidemia [14]. Esta, para que possa ser classificada como tal, deve representar um aumento expressivo no número de casos em diversas regiões, como por exemplo um município inteiro, um estado e até mesmo países. Como exemplo recente mais preocupante, tem-se a epidemia de Ebola ocorrida no oeste da África.

Há, ainda, os casos de endemia, quando uma doença ocorre com incidência significativa em alguma região. Aqui, pode-se citar casos de dengue que ocorrem em determinadas regiões do Brasil sempre que começa o período de chuvas, que é propício ao aparecimento da doença devido ao maior acúmulo de água, aumentando os locais onde os agentesAedes aegypti podem procriar.

A pandemia é o pior dos casos, quando uma nova doença se espalha a nível mundial, sendo marcada por epidemias em todo o globo. Como exemplos, pode-se citar a AIDS que, apesar de ser uma doença que está diminuindo graças às políticas de intervenções realizadas a nível global e à conscientização da população, chegou a infectar 10 milhões de indivíduos nas primeiras décadas desde seu descobrimento [15]. Outro caso muito importante foi a gripe H1N1, que em 2009 foi declarada como pandemia pela Organização Mundial da Saúde (OMS), tendo sido confirmadas infecções em 74 países e territórios [16].

Em todos os casos de doenças citados, desde surtos a epidemias, a eficiência da resposta ao aparecimento das doenças sempre foi crucial para sua extinção ou ao menos diminuição. Porém, as formas de prevenção tais como a vacinação, a eliminação do agente transmissor e, no caso da AIDS e do Ebola, a proteção contra o contato com o agente infeccioso, ainda são as melhores maneiras de se eliminar uma doença. No caso do Ebola, houve críticas à OMS pela ineficiência em lidar com a doença durante os surtos iniciais, o que foi posteriormente admitido pela 

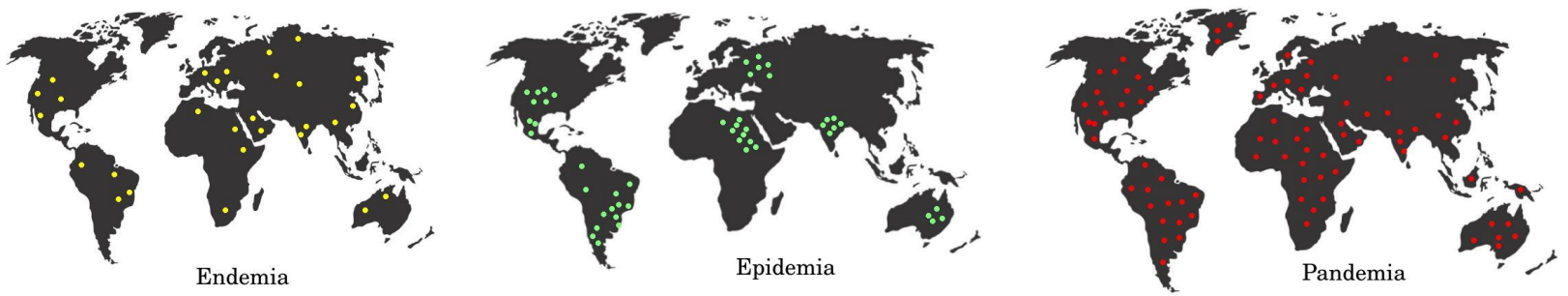

Figura 2.2: Doenças endêmicas, epidemias e pandemia.

própria organização [17]. Aqui, é importante atentar para o fato de que mesmo assuntos tão importantes como doenças graves são, muitas vezes, tratados sob um viés político-econômico muito forte, em detrimento do viés humanitário. Doenças como as que acometem países subdesenvolvidos e em desenvolvimento (Ebola nos países Africanos e Zika no Brasil, respectivamente) não recebem a atenção e o tratamento necessários [18, 19], sendo justamente as comunidades mais pobres aquelas mais vulneráveis a epidemiais. Nestes locais, muitas vezes o saneamento básico é precário e boa parte da população vive em meio ao lixo, com esgoto a céu aberto, e não tem hábitos de higiene adequados pelo simples fato do local onde vivem não possuir nem uma estrutura para tal. Nesses países, em que parte da população está em um nível de pobreza extremo, não há nem mesmo o que comer. Os indivíduos se tornam então hospedeiros ideais para proliferação de bactérias, vírus e parasitas, pois seu sistema imunológico é fraco. Nesses países, os estudos científicos, os diferentes modelos e previsões que surgem todos os anos para tratar doenças não são implementados.

Além desses problemas de ordem sócio-econômica, há que se comentar uma questão de crescente importância no mundo da saúde pública: o movimento antivacina. Com razões infundadas tais como "autismo causado por vacinas", ou a falta de credibilidade na eficiência delas, milhares de pessoas não vacinam seus filhos e nem a si mesmos. E esta atitude antivacina tem feito com que doenças como o sarampo [20], altamente contagiosa e causada pelo vírus Measles morbillivirus, cujos casos haviam sido erradicados em diversos países graças à vacinação, estejam retornando. Um caso icônico foi o de uma família francesa, composta de mãe, pai e criança de 5 anos, que foi tida como suspeita de haver reintroduzido o sarampo na Costa Rica [21] onde, desde 2004, não havia mais casos. Isto mostra a seriedade do problema 
do movimento antivacina.

Finalmente, pode-se concluir que abordar doenças graves, independentemente da região onde elas ocorrem, pelo tratamento efetivo dos infectados, de estudos da dinâmica de doenças, da análise das políticas de intervenção mais eficientes e da conscientização da população sobre a importância das vacinas e outras formas de controle, é de extrema importância para que doenças conhecidas e altamente contagiosas não reapareçam ou para se evitar que as novas doenças que venham a surgir se espalhem. 


\section{Modelagem matemática de epidemias}

Observa-se na natureza muitos casos em que determinadas formas de vida acabam servindo como hospedeiras para outras formas de vidas (e os vírus), que são os parasitas, tais como bactérias e fungos. As doenças infecciosas são casos bem conhecidos da relação hospedeiro-parasita e acredita-se que estejam presentes na humanidade desde seus estágios iniciais [22], visto que macacos eram hospedeiros de diversos vírus equivalentes aos encontrados no ser humano.

Com a luta pela sobrevivência da uma espécie sendo o carro chefe da sua evolução, o ser humano precisa aprender a lidar com as doenças infecciosas, observando padrões e aprendendo a evitar atitudes que podem causar sua morte. Por exemplo, ao observar uma ligação entre a morte de indivíduos e beber água de uma certa região frequentada por determinados animais, um grupo busca outro local para beber água. Porém, levou muito tempo para que a humanidade se desenvolvesse a ponto de entender melhor as doenças infecciosas e começar a produzir os primeiros trabalhos contendo uma análise matemática a seu respeito.

Em 1760, Daniel Bernoulli fez um estudo matemático sobre a varíola [23], cujo propósito principal era o de analisar como a inoculação contra a doença seria benéfica. Em 1849, John Snow, considerado o pai da epidemiologia, mostrou, por uma análise de dados, que os casos de cólera em Londres em 1854 eram relacionados a uma fonte de água que, uma vez retirada, fez com que os casos diminuíssem [24].

Estudos como os realizados por Bernoulli e Snow mostraram a importância de se analisar rigorosamente a dinâmica de transmissão de patógenos e os dados 
sobre os casos de infecção, para que as causas e os riscos sejam avaliados e a melhor solução seja proposta. Com estes trabalhos, dá-se início ao desenvolvimento de uma modelagem matemática robusta para descrever epidemias que ainda hoje serve de base para muitos estudos em epidemiologia.

Neste capítulo, os modelos compartimentais homogêneos são introduzidos e exemplificados. Para tratar a questão da heterogeneidade presente nas relações entre os indivíduos, são abordados alguns modelos que consideram redes de contato com diferentes graus de conexão. É também abordado o modelo estocástico, que surge como uma tentativa de modelar epidemias para pequenas populações, situação na qual é razoável assumir um certo grau de incerteza sobre o número de infectados.

\subsection{Modelos compartimentais e determinísticos}

Na busca por uma maneira de quantificar e entender o comportamento de doenças, buscando responder a perguntas tais como "quantas pessoas serão infectadas por uma epidemia?", ou "qual fração da população é preciso vacinar para evitar uma epidemia?", surgem os modelos compartimentais determinísticos. Eles foram pela primeira vez desenvolvidos por Kermack e MacKendrick [25] em 1927 como uma forma de modelar doenças infecciosas. Desde então, têm sido utilizados em diversos estudos e aplicações em epidemiologia [26-28].

Estes modelos assumem que os indivíduos de uma população, de tamanho fixo ou variável, possam ser divididos em compartimentos, que são grandes o suficiente de modo que a mistura dos membros seja homogênea, possuindo a mesma taxa de contato - ou seja, os indivíduos da população têm a mesma chance de infectar qualquer outro. As condições de saúde podem ser dividas nos seguintes compartimentos: suscetíveis (S), expostos (E), infectados (I) e recuperados (R), que são os compartimentos mais comumente utilizados (Fig. 3.1). A soma total dos indivíduos em cada um dos compartimentos fornece o tamanho $N$ da população, e cada indivíduo é considerado estatisticamente independente.

Os indivíduos podem circular entre os diferentes compartimentos por meio dos estágios pelos quais podem passar a partir do momento em que entram em contato com o patógeno, neste primeiro caso sendo considerados suscetíveis $(\mathrm{S})$. Os indivíduos expostos (E) entram nessa categoria quando estão infectados, porém 


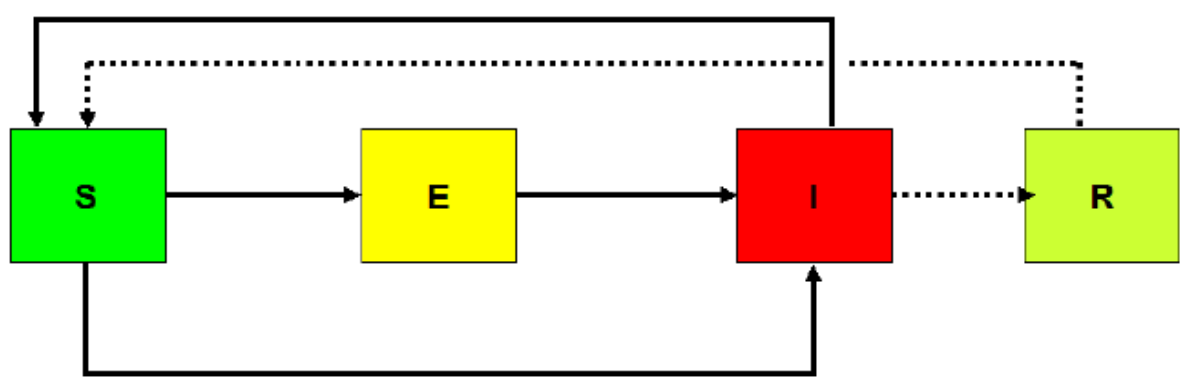

Figura 3.1: a) Representação esquemática do modelo compartimental contendo indivíduos que podem ser classificados como suscetíveis (S), expostos (E), infectados (I) e recuperados (R). A dinâmica do processo se dá de tal forma que indivíduos suscetíveis podem se tornar expostos através do contato com infectados. Uma vez adquirida a doença, tornam-se infectados. Em alguns casos possíveis (representados pelas linhas pontilhadas), os indivíduos podem se recuperar da doença, tornando-se resistentes ou imunes e entrando na classe de recuperados. Indivíduos recuperados podem, no entanto, tornar-se suscetíveis novamente, como ocorre no caso de doenças às quais se adquire imunidade apenas por um período (gripe, por exemplo).

não são infecciosos. Assim que adquirem a doença e podem transmiti-la, tornamse infectados (I). Para que um indivíduo seja considerado como recuperado (R), ele pode ter adquirido imunidade à doença ou pode ter sido retirado da população considerada, seja pela morte ou uma mudança de região, por exemplo.

O fluxo de um compartimento a outro é quantificado pela variação temporal dos indivíduos que nele estão, sendo que a mudança no estado de saúde contribui negativamente para a dinâmica do respectivo compartimento se ela implicar saída dele e entrada em outro compartimento, e positivamente, caso haja entrada no compartimento considerado. Esta mudança de estado de saúde dos indivíduos leva em conta também taxas com as quais os deslocamentos entre compartimentos ocorrem, como por exemplo taxa de infecção e taxa de cura.

Modelos compartimentais podem se tornar tão complicados quanto se queira. Por exemplo, ao dividir a população em diversos subgrupos tais como suscetíveis, expostos, em quarentena, infectados, isolados, mortos e curados, com diferentes taxas de transição de um grupo para outro, acaba-se com um modelo que possui diversas equações diferenciais. Um exemplo é o modelo desenvolvido por S. Del Valle et al para tratar a varíola [29] (Fig.3.2). Além disso, alguns modelos podem incluir taxas de mudança de um compartimento a outro que sejam variáveis no tempo, 


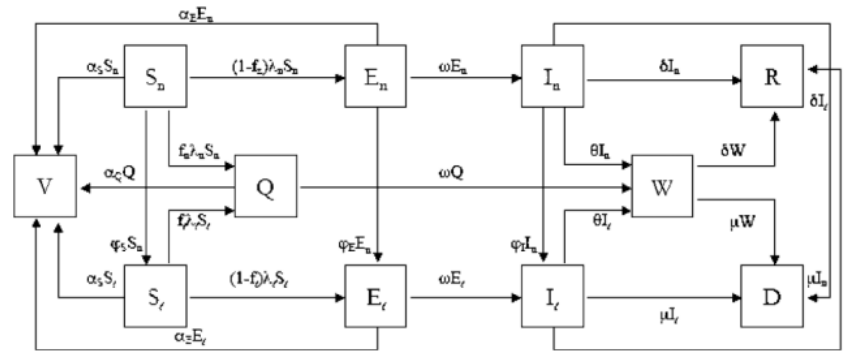

a)
$\dot{V}=\alpha_{S}\left(S_{n}+S_{\ell}\right)+\alpha_{E}\left(E_{n}+E_{\ell}\right)+\alpha_{Q} Q$,

$\dot{S}_{n}=-\lambda_{n} S_{n}-\left(\varphi_{S}+\alpha_{S}\right) S_{n}$,

$\dot{S}_{\ell}=-\lambda_{\ell} S_{\ell}+\varphi_{S} S_{n}-\alpha_{S} S_{\ell}$,

$\dot{Q}=f_{n} \lambda_{n} S_{n}+f_{\ell} \lambda_{\ell} S_{\ell}-\left(\omega+\alpha_{Q}\right) Q$

$\dot{E}_{n}=\left(1-f_{n}\right) \lambda_{n} S_{n}-\left(\varphi_{E}+\omega+\alpha_{E}\right) E_{n}$,

$\dot{E}_{\ell}=\left(1-f_{\ell}\right) \lambda_{\ell} S_{\ell}+\varphi_{E} E_{n}-\left(\omega+\alpha_{E}\right) E_{\ell}$

$\dot{I}_{n}=\omega E_{n}-\left(\varphi_{I}+\mu+\delta+\theta\right) I_{n}$,

$\dot{I}_{\ell}=\omega E_{\ell}+\varphi_{I} I_{n}-(\mu+\delta+\theta) I_{\ell}$

$\dot{W}=\theta\left(I_{n}+I_{\ell}\right)+\omega Q-(\mu+\delta) W$,

$\dot{R}=\delta\left(I_{n}+I_{\ell}+W\right)$,

$\dot{D}=\mu\left(I_{n}+I_{\ell}+W\right)$.

b)

Figura 3.2: a) Representação esquemática do modelo compartimental, no qual os indivíduos são divididos em várias subclasses. b) Equações diferenciais derivadas do modelo compartimental. Figura retirada do trabalho de S. Del Valle et al, Mathematical Biosciences 195 (2005).

e este fator, juntamente com a quantidade de equações diferenciais que o modelo possui, aumenta o nível de dificuldade de solução do problema.

\subsection{Refinamentos do modelo compartimental}

Os modelos compartimentais, por mais variáveis que eles possam conter, são determinísticos e assumem homogeneidade da população. Portanto, não levam em conta fatores de heterogeneidade que podem ser introduzidos pela natureza randômica da interação entre indivíduos, por fatores ambientais, tais como lugares mais isolados e regiões onde o saneamento básico é precário e que portanto vão ser atingidas de maneiras diferentes, ou mesmo pelo tamanho da população considerada. Desta forma, modelos determinísticos homogêneos acabam por não representar a realidade de alguns processos epidemiológicos, e torna-se necessário introduzir outros fatores.

Em relação à questão da estrutura heterogênea, foram propostas diversas maneiras de se introduzi-la em um modelo epidêmico. Há modelos que consideram taxas de contato heterogêneas dependentes de alguma função matemática [30], outros utilizam a modelagem por grafos [31-35], nas quais indivíduos são considerados como vértices de um grafo e possuem diferentes conexões, representadas por linhas pelas quais é possível transmitir o patógeno (figura 3.3). Esta abordagem permite 


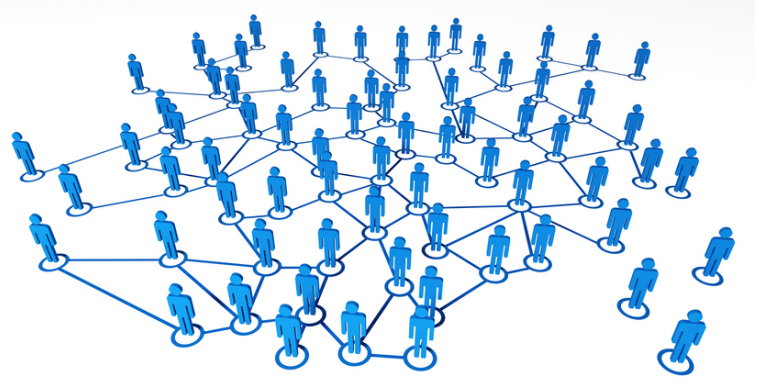

Figura 3.3: Representação esquemática de um grafo com diferentes graus de conectividade, no qual alguns indivíduos em vértices mais distantes são menos conectados do que aqueles no centro. Informações sobre a estrutura do grafo podem ser retiradas de censos populacionais, por exemplo.

incluir diferentes graus de complexidade de interações interpessoais e podem refletir a realidade de um mundo altamente conectado como o que vivemos, onde indivíduos de um determinado país podem viajar e introduzir alguma doença em outro país. Porém, a modelagem por grafos exigiria o conhecimento específico da estrutura da população, o que é uma informação difícil de obter precisamente. Portanto, na prática são feitas aproximações e estimativas baseadas em dados sobre a população que são possíveis de serem coletados. Um exemplo é modelagem usando grafos para analisar a transmissão da AIDS [36], que conta não só com a estrutura das relações sexuais como também as de compartilhamento de agulhas (seja para uso de drogas ou tatuagens), cujos dados são difíceis de obter empiricamente e requerem aproximações.

O caso de pequenas populações também não é contemplado pelas equações compartimentais determinísticas, uma vez que para estes casos pode-se esperar que haja incertezas quanto ao número de infectados. Portanto, quando são tratadas doenças em pequenos vilarejos, famílias de comunidades isoladas ou mesmo os estágios iniciais de um surto [37], no qual o número de pessoas atingidas inicialmente é pequeno, é preciso trabalhar com valores médios. Modelos epidêmicos estocásticos $[38,39]$ surgem então como uma tentativa de tornar a modelagem o mais próxima possível da realidade, incluindo no modelo probabilidades de transição de uma categoria a outra.

Pode-se dizer, portanto, que os os modelos compartimentais, apesar de terem 
surgido com a proposta de modelarem populações grandes e homogêneas, quando aliados a novas técnicas são capazes de descrever as diferentes condições de heterogeneidade da população considerada. A questão principal da modelagem de epidemias está em saber quando é necessário acrescentar complexidade aos modelos de modo a tornar as previsões mais robustas [40]. 


\section{4}

\section{Modelagem baseada em agentes}

No capítulo anterior, foram mostrados modelos matemáticos que podem ser usados para descrever a dinâmica de processos epidêmicos, cuja hipótese principal consiste na homogeneidade da população. Um processo epidêmico bastante utilizado para tratar doenças que não conferem imunidade a longo prazo é o Suscetível - Infectado - Suscetível (SIS), no qual os indivíduos de uma população são classificados como suscetíveis a uma doença ou infectados, e podem circular entre estas duas classificações. Doenças sexualmente transmissíveis possuem, em sua maioria, comportamento disseminatório bem representado pelo modelo SIS, pois um indivíduo encontra-se suscetível a uma determinada doença transmitida sexualmente e, a partir do momento em que entra em contato com ela, pode tornar-se infectado. Como algumas delas não conferem imunidade permanente, tais como a sífilis, causada pela bactéria Treponema pallidium, um indivíduo, uma vez curado, pode voltar a contraí-la.

Para as DST, a transmissão dos patógenos ou infecção ocorre somente quando há relação sexual. A suposição natural para esta classe de doenças inclui a finitude do número de parceiros sexuais, bem como a separação da população em grupos, de acordo com suas respectivas orientações sexuais. Portanto, é importante notar que nestes casos as interações entre indivíduos não são bem representadas por uma rede de conexões homogênea, como ocorre na modelagem de doenças transmitidas por vias aéreas. É evidente que, para as DST, a estrutura de contatos é heterogênea, o que não é contemplado nos modelos compartimentais determinísticos, que assumem a equivalência entre todos os indivíduos de uma população. Um indivíduo possui interações sociais/biológicas características e há também os grupos de risco. Desta 
forma, um modelo matemático para DST deve levar em consideração as diferenças das relações interpessoais da população, a fim de melhor prever a transmissão do patógeno.

Fatores estocásticos também são de grande relevância, principalmente quando busca-se descrever a dinâmica de um patógeno em pequenas populações, nas quais as incertezas em relação ao número de infectados terão papel importante no processo de análise da propagação da doença.

Buscando usar como base o modelo SIS para descrever a dinâmica de doenças como DST, gripe comum e influenza, este capítulo introduz e descreve o modelo compartimental SIS, que assume a homogeneidade da população considerada. Como forma de introduzir a heterogeneidade, a modelagem baseada em agentes é descrita. Por fim, um modelo estocástico para o processo SIS usando modelagem baseada em agentes é desenvolvido, permitindo analisar a propagação das doenças em pequenas populações.

\subsection{Modelo compartimental}

Foi dito que modelos compartimentais dividem a população considerada em compartimentos de acordo com seu estado de saúde. Um dos modelos mais simples é o SIS [41], que é estudado nesta dissertação, no qual os indivíduos são divididos em dois compartimentos: os suscetíveis, que podem adquirir uma doença, e os infectados, que possuem a doença, podem transmiti-la e serem curados. A circulação ocorre apenas entre estes dois compartimentos, portanto este modelo contempla doenças que não conferem imunidade após a infecção, como é o caso de doenças sexualmente transmissíveis tais como gonorréia, sífilis e AIDS.

As equações que regem o modelo SIS clássico consideram que o tamanho da população seja constante, ou seja, $S(t)+I(t)=N$, onde $S(t)$ é o número de indivíduos suscetíveis no tempo $t, I(t)$ o número de indivíduos infectados no tempo $t$ e $N$, o número total de pessoas. Em média, o número de nascimentos e mortes são iguais nessa população. Além disso, uma suposição importante é a de que todos os indivíduos estão igualmente misturados, de modo que a chance de contato é igual para todos.

É fácil concluir que a variação no número de infectados depende da interação 


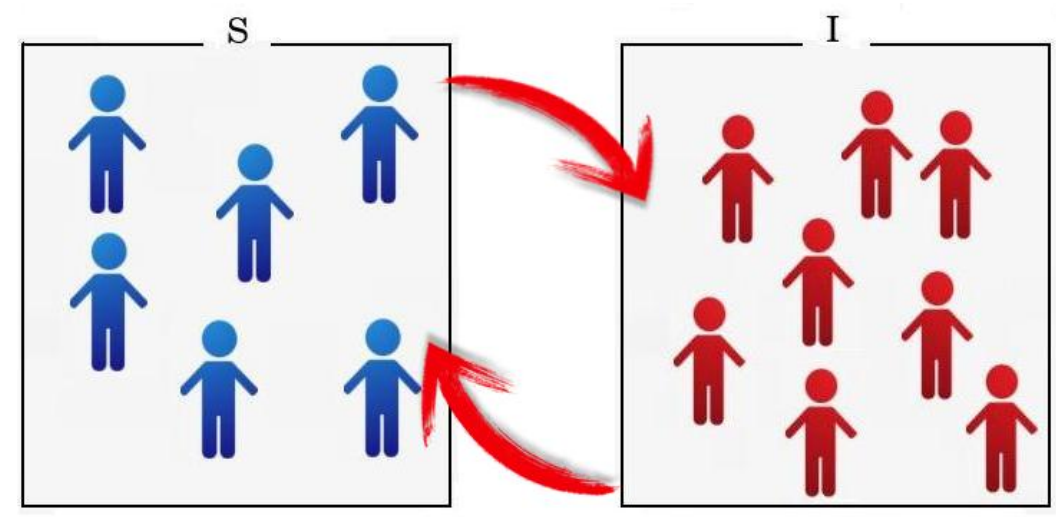

Figura 4.1: Modelo SIS compartimental, onde ocorre a troca entre compartimentos com indivíduos suscetíveis (azul) e infectados (vermelho).

entre suscetíveis e infectados, aumentando com esta, pois, ao contato de um indivíduo infectado com um não-infectado, pode ocorrer a contaminação e, neste caso, o indivíduo sai da categoria suscetível e entra na catoria infectado. Além disso, a cura de um indivíduo infectado contribui para a diminuição deste compartimento. Já a variação no número de suscetíveis diminui com a interação suscetível-infectado e aumenta com a cura dos indivíduos infectados, de modo que as equações são dadas por:

$$
\begin{aligned}
& \frac{d S}{d t}=-\frac{\alpha S I}{N}+\gamma I, \\
& \frac{d I}{d t}=\frac{\alpha S I}{N}-\gamma I,
\end{aligned}
$$

onde $\alpha / N$ é a taxa de transmissão, que representa a probabilidade de transmissão da doença, e $\gamma$ a taxa de cura, determinada pela duração média da doença.

Divindo a Eq. (4.2) por $N$ e usando a conservação do número de pessoas que diz que $S=N-I$, tem-se:

$$
\frac{1}{N} \frac{d I}{d t}=\alpha\left(1-\frac{I}{N}\right) \frac{I}{N}-\gamma \frac{I}{N} .
$$

Pode-se definir $I / N=\rho$ como a densidade de infectados. Deste modo, a Eq. (4.3) pode ser rearranjada:

$$
\frac{d \rho}{d t}=\alpha(1-\rho) \rho-\gamma \rho .
$$

Aqui, define-se $\rho_{e q}=1-\gamma / \alpha$ como um valor de equilíbrio, para o qual deve-se ter $\gamma / \alpha>0$ já que as taxas de infecção e cura são positivas, o que implica $\rho_{e q}<1$ 
(o caso $\gamma / \alpha=0$ fornece o modelo SI, onde eventualmente todo indivíduo se torna infectado). Com isso, a Eq. (4.4) pode ser escrita como

$$
\frac{d \rho}{d t}=\alpha\left(\rho_{e q}-\rho\right) \rho
$$

No estado estacionário $(t \rightarrow \infty)$, o número de infectados tende a um desses valores $\rho^{*}=0$ (extinção da doença) e $\rho^{*}=\rho_{e q}$. As condições para que cada situação ocorra são tais que:

i) Se $\rho_{e q}<0$, implicando $\gamma>\alpha$, então $\rho(t \rightarrow \infty) \rightarrow 0$, o que significa que $I(t \rightarrow \infty) \rightarrow 0$ e $S(t \rightarrow \infty) \rightarrow N$. Neste caso, independentemente da condição inicial $\rho(0)$, tem-se a extinção da doença, dado que a taxa de cura é maior do que a de infecção.

ii) Se $\rho_{e q} \geq 0$, implicando $\gamma<\alpha$, então $\rho(t \rightarrow \infty) \rightarrow \rho_{e q}$, o que significa que $I(t \rightarrow \infty) \rightarrow N \rho_{e q}$ e $S(t \rightarrow \infty) \rightarrow N\left(1-\rho_{e q}\right)$. Neste caso, independentemente da condição inicial $\rho(0)$, o número de infectados tende a um valor de equilíbrio não nulo.

Com isso, tem-se um modelo determinístico que descreve a dinâmica do espalhamento da doença para os casos mais simples nos quais a hipótese de que a população seja homogênea é válida.

\subsection{Modelo baseado em agentes}

Uma forma de lidar com a heterogeneidade na modelagem matemática, seja ela advinda das particularidades de cada indivíduo considerado, seja por conta do ambiente no qual os indivíduos se encontram, é pela modelagem baseada em agentes [42]. Tal modelagem é uma técnica que consiste em descrever uma população ou um sistema biológico definindo-se os seguintes componentes:

- Agentes: são os indivíduos de uma população (ou componentes de sistemas biológicos [43]). A cada agente são associados determinados estados nos quais pode-se encontrá-los. A mudança de estado de um agente ocorre pela interação com outros agentes (no modelo SIS, infecção por contato) ou por um fator externo (no modelo SIS, cura).

- Grafo de interação: é o grafo subjacente que define como os agentes conectam-se e interagem entre si. 
- Ambiente: é o local no qual os agentes existem e onde se relacionam (pode ser uma cidade, uma vilarejo, uma casa).

- Regras de interação: são regras que definem o estado dos agentes, podendo ser relacionadas à interação com os vizinhos ou a um fator do ambiente. Nas situações nas quais um agente se depara com a ocorrência dos requerimentos de uma regra, seu estado muda. Como exemplo, para o modelo SIS poderse-ia considerar as seguintes regras: agente suscetível encontra com ao menos um vizinho infectado $\Rightarrow$ mudança no estado de saúde para infectado; agente infectado não possui nenhum vizinho próximo infectado $\Rightarrow$ mudança no estado de saúde para suscetível.

- Escala de tempo: o tempo total durante o qual o sistema é simulado é dividido em passos nos quais os agentes interagem entre si e com o ambiente, e terão seus estados de saúde alterados conforme as regras estabelecidas.

A modelagem baseada em agentes permite, portanto, que sejam descritos sistemas biológicos mais próximos da realidade, onde o ambiente passa a ser um fator importante e o individual deve ser levado em conta para descrever o sistema biológico. Esta modelagem é, devido aos seus aspectos, base para a formulação do modelo SIS que será desenvolvida nesta dissertação.

\subsection{Espaço vetorial, operadores e matriz de transição}

Queremos aqui explorar a formulação matemática desenvolvida por Nakamura et al [44], e investigada de maneira mais aprofundada em nossos estudos para expor os novos resultados [45]. De modo resumido, essa formulação leva em conta a estocasticidade do processo de transmissão de doenças, acomodando inclusive os efeitos devido à heterogeneidade da população. No que segue, detalhamos tal formulação para o modelo SIS para facilitar a reprodução e generalização de nossos resultados.

Considere o processo de transmissão de um patógeno que segue o modelo SIS numa população composta por $N$ indivíduos. Observa-se que estes são univocamente indentificados por um índice inteiro $k=1,2, \ldots, N$ e que as interações entre os diferentes indivíduos são descritas pela matriz de adjacência, $A$. 
O estado de saúde do $k$-ésimo indivíduo é descrito pela variável binária $n_{k}$ :

$$
n_{k}=\left\{\begin{array}{ll}
1, & \text { se infectado } \\
0, & \text { se suscetível }
\end{array} .\right.
$$

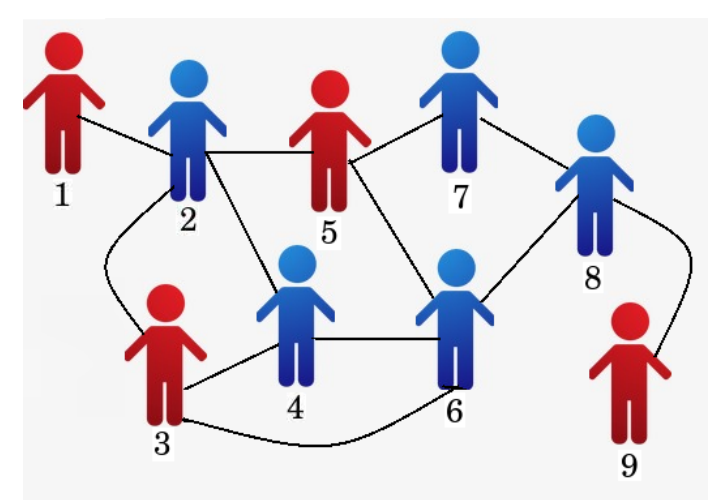

Figura 4.2: Indivíduos infectados e suscetíveis identificados por um indíce inteiro e cuja interação é representada pelas linhas conectando cada um deles.

Deste modo, para cada instante de tempo, essa população de tamanho $N$ apresenta um certo número de indivíduos infectados e outros suscetíveis, número este que varia conforme mais infecções ou curas ocorrem. Isto fornece diferentes configurações $|\mu\rangle$ desses $N$ indivíduos, que são representadas por:

$$
|\mu\rangle=\left|n_{1} n_{2} \ldots n_{N}\right\rangle,
$$

onde $\mu=n_{1} 2^{0}+n_{2} 2^{1}+\ldots+n_{N} 2^{N-1}$, indo de 0 até $2^{N}-1$. Para $N=3$, a Figura 4.3 mostra todas as configurações possíveis.

É importante frizar que, em um determinado instante, pode-se observar a população em qualquer uma das possíveis configurações. Porém, algumas configurações ocorrem com maior probabilidade do que outras. Por exemplo, dada a evolução da doença na população, a configuração na qual não há indivíduos infectados é pouco provável. Portanto, a avaliação da evolução temporal do sistema requer a combinação linear de todas as configurações, ponderadas por suas respectivas probabilidades instantâneas. Assim, define-se o vetor $|P(t)\rangle$ como

$$
|P(t)\rangle=\sum_{\mu} P_{\mu}(t)|\mu\rangle,
$$

onde o coeficiente $P_{\mu}(t)$ representa a probabilidade no instante de tempo $t$ de observação da configuração $|\mu\rangle$. Considere o seguinte exemplo: no instante $t=0$, há 


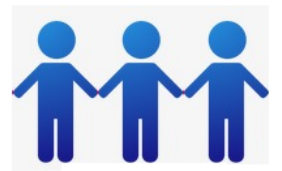

$|0\rangle=|000\rangle$

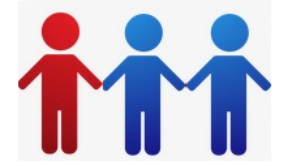

$|1\rangle=|100\rangle$

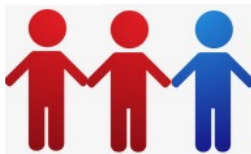

$|3\rangle=|110\rangle$

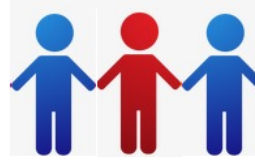

$|2\rangle=|010\rangle$

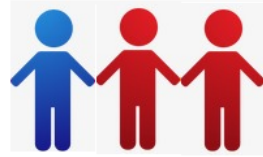

$|6\rangle=|011\rangle$

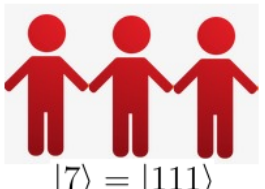

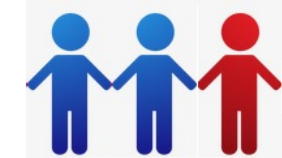

$|4\rangle=|001\rangle$

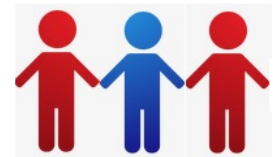

$|5\rangle=|101\rangle$

Figura 4.3: Todas as 8 configurações possíveis para $N=3$. Em vermelho, indivíduos infectados; em azul, suscetíveis.

apenas um indivíduo infectado, que possui conexão com alguns outros indivíduos da rede de interação considerada. A probabilidade de encontrar, nos estágios inicias da propagação do patógeno, a configuração onde todos estão infectados é baixa. No entanto, conforme a doença se espalha consideravelmente, mais indivíduos vão sendo infectados, cada um deles podendo infectar outras pessoas. Assim, com o decorrer do tempo aumenta-se a probabilidade de encontrar o sistema na configuração em que todos estão infectados.

O vetor $|P(t)\rangle$ recebe o nome de vetor probabilidade e descreve o sistema. Suas propriedades são o vínculo da probabilidade total $\sum_{\mu} P_{\mu}(t)=1$ e a conservação da probabilidade total, $\frac{d}{d t} \sum_{\mu} P_{\mu}(t)=0$.

Considere, agora, todas as ações que podem ocorrer a um sistema. Elas representam uma sequência de transformações, cada uma delas ocorrendo em um determinado intervalo de tempo $\delta t$. É importante salientar que considera-se este intervalo de tempo de tal forma que ocorra apenas uma mudança por vez no sistema (processo poissônico independente), ou seja, pode ocorrer apenas uma infecção, ou uma cura ou nada, e o sistema permanece como estava. Logo, um intervalo de tempo de um mês não seria adequado, pois várias mudanças podem ocorrer, po- 
rém $\delta t$ da ordem de microssegundos também não seria uma boa escolha, pois não observaríamos nada.

Estas transformações levam o sistema de uma configuração $|\mu\rangle$ para outra $\left|\mu^{\prime}\right\rangle$, e formam um processo de Markov, que é um processo aleatório no qual é possível fazer previsões do futuro baseadas apenas no presente, sem a dependência do passado (também chamado de processo sem memória). O conjunto de transformações entre as diferentes configurações é representado pela matriz de transição $\hat{T}$, que contempla as seguintes possibilidades:

i) um indivíduo infectado é curado, se tornando susceptível;

ii) um indivíduo susceptível é infectado pelo contato com alguém que possui a doença;

iii) nada ocorre.

Para quantificar as transições presentes no modelo SIS é importante atentar aos elementos que realizam as mudanças citadas. Estes elementos executam ações sobre configurações do sistema, ou seja, agem sobre um espaço de estados levandoo a outro e são, portanto, operadores que agem no espaço vetorial formado pelo conjunto de $2^{N}$ vetores $\{|\mu\rangle\}$ que geram o espaço de Hilbert, formando uma base ortonormal, sendo sua ortonormalidade expressa por $\langle\nu \mid \mu\rangle=\delta_{\mu, \nu}$.

Considere o operador:

$$
\hat{\sigma}_{k}^{z}\left|n_{1} n_{2} \ldots n_{k} \ldots n_{N}\right\rangle=\left(2 n_{k}-1\right)\left|n_{1} n_{2} \ldots n_{k} \ldots n_{N}\right\rangle
$$

ele age sobre uma configuração retornando a própria configuração multiplicada por +1 se o indivíduo do $k$-ésimo vértice estiver infectado ou por -1 , se tal indivíduo for susceptível. Define-se também o operador número:

$$
\hat{n}_{k}\left|n_{1} n_{2} \ldots n_{k} \ldots n_{N}\right\rangle=n_{k}\left|n_{1} n_{2} \ldots n_{k} \ldots n_{N}\right\rangle
$$

que retorna 0 (susceptível) ou 1 (infectado) dependendo do estado de saúde do indivíduo. Assim, o operador número total de infectados é dado por $\hat{n}=\sum_{k} \hat{n}_{k}$. Como exemplo, considere a configuração $|3\rangle=|1100\rangle$. Aplicando o operador número total de infectados a ela, tem-se: $\hat{n}|3\rangle=\sum_{k=1}^{4} \hat{n}_{k}|1100\rangle=\hat{n}_{1}|1100\rangle+\hat{n}_{2}|1100\rangle+$ $\hat{n}_{3}|1100\rangle+\hat{n}_{4}|1100\rangle=1|1100\rangle+1|1100\rangle+0|1100\rangle+0|1100\rangle=2|3\rangle$. Desta forma, o número total de infectados é dado pela soma dos valores $n_{k}$ fornecidos quando da aplicação do operador $\hat{n}_{k}$ à configuração. 
A configuração $|\mu\rangle$ é autovetor de ambos os operadores $\hat{\sigma}_{k}^{z}$ e $\hat{n}_{k}$. Portanto, eles não descrevem transições entre diferentes configurações. Desta forma, para que se possa definir eventos de cura ou infecção de um indivíduo, é necessário introduzir dois novos operadores cujas ações são definidas por:

$$
\begin{aligned}
\hat{\sigma}_{k}^{+}\left|n_{1} n_{2} \ldots 0_{k} \ldots n_{N}\right\rangle & =\left|n_{1} n_{2} \ldots 1_{k} \ldots n_{N}\right\rangle, \\
\hat{\sigma}_{k}^{-}\left|n_{1} n_{2} \ldots 1_{k} \ldots n_{N}\right\rangle & =\left|n_{1} n_{2} \ldots 0_{k} \ldots n_{N}\right\rangle,
\end{aligned}
$$

e nulo para outros casos. O operador $\hat{\sigma}_{k}^{+}$age em uma configuração mudando o estado do $k$-ésimo indivíduo de susceptível para infectado, de modo que será chamado de operador infecção. Caso este indivíduo já se encontre infectado, esta ação retorna o valor nulo. Já o operador $\hat{\sigma}_{k}^{-}$age no sentido contrário, mudando o estado do $k$-ésimo indivíduo de infectado para susceptível, de modo que será chamado de operador cura. De maneira análoga, a ação do operador cura retorna valor nulo caso o indivíduo já se encontre no estado suscetível.

Todos os operadores $\hat{\sigma}$ definidos satisfazem as seguintes relações de comutação:

$$
\begin{aligned}
{\left[\hat{\sigma}_{k}^{z}, \hat{\sigma}_{k}^{ \pm}\right] } & = \pm 2 \hat{\sigma}_{k}^{ \pm}, \\
{\left[\hat{\sigma}_{k}^{+}, \hat{\sigma}_{k}^{-}\right] } & =\hat{\sigma}_{k}^{z},
\end{aligned}
$$

que são a não-comutação dos operadores $\hat{\sigma}_{k}^{z}$ e $\hat{\sigma}_{k}^{ \pm}$e não-comutação dos operadores de infecção e cura para um mesmo vértice.

É fácil perceber que os operadores de cura e infecção comutam para vértices diferentes, pois a ação de um não afeta a do outro, uma vez que eles agem em indivíduos distintos, de modo que neste caso não existe nenhuma relação entre eles:

$$
\begin{gathered}
{\left[\hat{\sigma}_{k}^{+}, \hat{\sigma}_{j}^{-}\right]=0,} \\
{\left[\hat{\sigma}_{k}^{z}, \hat{\sigma}_{j}^{+}\right]=0,} \\
{\left[\hat{\sigma}_{k}^{z}, \hat{\sigma}_{j}^{-}\right]=0 .}
\end{gathered}
$$

Uma vez definidos os operadores, a matriz de transição pode ser finalmente escrita:

$$
\hat{T}=\hat{1}-\frac{\alpha \delta t}{N} \sum_{j, k=0}^{N-1} A_{j k}\left(1-\hat{n}_{k}\right) \hat{n}_{j}-\gamma \delta t \sum_{k=0}^{N-1} \hat{n}_{k}+\frac{\alpha \delta t}{N} \sum_{j, k=0}^{N-1} A_{j k} \hat{\sigma}_{k}^{+} \hat{n}_{j}+\gamma \delta t \sum_{k=0}^{N-1} \hat{\sigma}_{k}^{-},
$$


onde $\hat{1}$ é a matriz diagonal unitária, $\delta_{j k}$ é o delta de Kroenecker, e $A_{j k}$ é a matriz de adjacência.

Analisando termo a termo da matriz de transição, tem-se:

i) $\gamma \delta t \sum_{k} \hat{\sigma}_{k}^{-}$- indivíduo infectado é curado, onde o operador de cura $\hat{\sigma}_{k}^{-}$age realizando a cura no $k$-ésimo indivíduo com probabilidade $\gamma \delta t$;

ii) $-\gamma \delta t \sum_{k} \hat{n}_{k}$ - indivíduo infectado permanece infectado, onde a ação do operador número retorna a mesma configuração. Probabilidade complementar de não ocorrer transição;

iii) $\alpha / \delta t N \sum_{j, k} A_{j k} \hat{\sigma}_{k}^{+} \hat{n}_{j}$ - indivíduo susceptível é infectado pelo contato, onde $\hat{n}_{j}$ verifica se há um infectado no vértice $j$, o operador infecção $\hat{\sigma}_{k}^{+}$age realizando a infecção no indivíduo do $k$-ésimo vertice e a matriz de adjacência $A_{j k}$ define a estrutura da conexão entre os indivíduos do $j$-ésimo e $k$-ésimo vértices;

iv) $-\alpha / \delta t N \sum_{j, k} A_{j k}\left(1-\hat{n}_{k}\right) \hat{n}_{j}$ - indivíduo susceptível permanece susceptível. Novamente, a ação dos operadores número retorna a mesma configuração e tem-se uma probabilidade complementar de não ocorrer transição.

A conservação de probabilidade restringe os elementos de $\hat{T}$ a serem nãonegativos com $\sum_{n, m} T_{n, m}=1$.

A matriz de transição atua sobre o vetor probabilidade, expressando todas as mudanças entre estados que podem ser realizadas pelo sistema. Isso equivale à seguinte equação de evolução temporal:

$$
\hat{T}|P(t)\rangle=|P(t+\delta t)\rangle
$$

de modo que a matriz de transição é o operador de evolução temporal do sistema. Nota-se que ela não é Hermitiana, uma vez que, para apresentar esta propriedade, a transição entre duas configurações $|\mu\rangle \leftrightarrows|\nu\rangle$ deveria ter a mesma probabilidade nos dois sentidos, o que não ocorre.

Para $\delta t \rightarrow 0$, a Eq. (4.18) deixa de ser um processo discretizado e passa a ser um processo que ocorre de maneira contínua no tempo. Assim sendo, procura-se escrever uma equação mestra [46] que descreva a evolução temporal de um sistema modelado em termos de combinações probabilísticas (vetor probabilidade, no presente caso) e cuja mudança entre diferentes estados seja determinada por uma matriz de transição. Para tanto, faz-se uma expansão em série de potências da Eq. (4.18) 
até primeira ordem para $\delta t$ :

$$
\begin{aligned}
& \hat{T}|P(t)\rangle=|P(t)\rangle+\delta t \frac{d|P(t)\rangle}{d t}+O\left(\delta t^{2}\right) \\
& \frac{d|P(t)\rangle}{d t}=-\frac{\hat{1}-\hat{T}}{\delta t}|P(t)\rangle \\
& \frac{d|P(t)\rangle}{d t}=-\hat{H}|P(t)\rangle,
\end{aligned}
$$

onde $\hat{H} \equiv(\hat{1}-\hat{T}) / \delta t$ é definido como sendo o gerador de translações temporais do sistema, assim nomeado, pois para cada tempo $\delta t$ considerado, este operador leva o sistema de um vetor de probabilidade a outro, gerando uma translação de uma configuração a outra.

Ao projetar o vetor probabilidade do lado esquerdo da Eq. (4.19) na base $|\mu\rangle$, pode-se escrevê-la da seguinte forma:

$$
\frac{d P_{\mu}}{d t}=-\langle\mu|\hat{H}| P(t)\rangle=-\sum_{\nu} H_{\mu \nu} P_{\nu}(t)
$$

onde $H_{\mu \nu} \equiv\langle\mu|\hat{H}| \nu\rangle$ e o vetor probabilidade do lado esquerdo foi escrito como $|P(t)\rangle=\sum_{\nu} P_{\nu}(t)|\nu\rangle$.

Conforme mostra a Eq. (4.17), a matriz de transição é do tipo $\hat{T}=\hat{1}-\hat{B}$. Portanto, pela definição de $\hat{H}$ tem-se que $\hat{H}=\hat{B} / \delta t$, resultando em

$$
\hat{H}=\frac{\alpha}{N} \sum_{j, k} A_{j k}\left(1-\hat{n}_{k}\right) \hat{n}_{j}+\gamma \sum_{k} \hat{n}_{k}-\frac{\alpha}{N} \sum_{j, k} A_{j k} \hat{\sigma}_{k}^{+} \hat{n}_{j}-\gamma \sum_{k} \hat{\sigma}_{k}^{-} .
$$

Finalmente, dadas as Eqs. (4.19) e (4.21), a solução para a evolução temporal do vetor probabilidade é:

$$
|P(t)\rangle=e^{-\hat{H} t}|P(0)\rangle .
$$

Tem-se, assim, um modelo matemático completo que descreve a evolução temporal das possíveis configurações existentes em um problema epidemiológico do tipo SIS. Ele leva em conta o fato de que não há determinismo, mas sim probabilidades para que ocorram certas configurações do sistema, além de ter embutida a matriz de adjacência, que, dependendo do seu formato, pode descrever sistemas nos quais os indivíduos possuem diferentes graus de conectividade.

\subsection{Flutuações}

Considerando-se uma população muito grande e homogênea, em que os indivíduos estão igualmente conectados, a equação compartimental (Eq. (4.5)) mostra-se 
uma boa aproximação para estudar o comportamento das doenças. Porém, para o caso de pequenas populações, como vilarejos e localidades rurais, mesmo quando as relações interpessoais sejam muito bem descritas pelo grafo completo, a finitude da população não elimina completamente o caráter estocástico do processo de disseminação. Assim sendo, procuramos neste capítulo encontrar uma nova equação que inclua os efeitos de flutuações gerados em pequenas populações.

No caso em que um indivíduo interage igualmente com todos os outros, temse a aproximação de campo médio, que é representada por um grafo completo, com a matriz de adjacência dada por $A_{j k}=1-\delta_{j k}$ (fig. 4.4). Realizando uma soma sobre todos os vértices $j, k$, pode-se escrever o gerador de translações temporais do modelo SIS da seguinte forma:

$$
\mathcal{H}=-\frac{\alpha}{N}\left(\hat{n}+\hat{S}^{+}\right) \hat{n}-\gamma \hat{S}^{-}+(\alpha+\gamma) \hat{n}
$$

onde definem-se os operadores globais $\hat{n} \equiv \sum_{k} \hat{n}_{k}$ e $\hat{S}^{ \pm} \equiv \sum_{k} \hat{\sigma}_{k}^{ \pm}$que são, respectivamente, o operador número e os operadores de levantamento (infecção) e de abaixamento (cura).

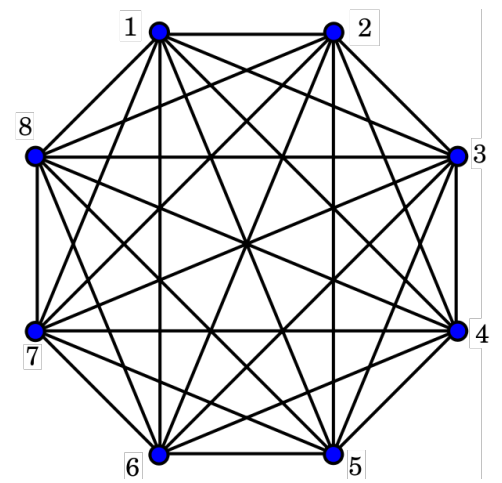

Figura 4.4: Representação de um grafo completo, com a matriz de adjacência dada por $A_{j k}=$ $1-\delta_{j k}$, no qual todos os indivíduos nos vértices de 1 a 8 estão conectados entre si. Imagem retirada da Wikipedia.

Para adicionar o caráter estocástico ao modelo compartimental, aplica-se a modelagem descrita na seção anterior, começando pela definição da densidade média de agentes infectados:

$$
\langle\rho(t)\rangle=\frac{1}{N} \sum_{\mu=0}^{2^{N}-1} \eta_{\mu} P_{\mu}(t)=\frac{1}{N} \sum_{\mu=0}^{2^{N}-1}\langle\mu|\hat{n}| P(t)\rangle,
$$

onde $\eta_{\mu} \equiv\left\langle\mu\left|\sum_{k} \hat{n}_{k}\right| \mu\right\rangle$ é o número de indivíduos infectados na configuração $|\mu\rangle$ e $k=1,2, \ldots, N$. Como exemplo, considere o vetor probabilidade $|P(t)\rangle=$ 
$(|100\rangle+|010\rangle+|001\rangle) / 3$, onde $N=3$ e assumem-se iguais as probabilidades $P_{\mu}(t)$ de cada configuração. Para este vetor, o número de indivíduos infectados em cada configuração é dado por: $\eta_{1}=\left\langle 100\left|\sum_{k=1}^{3} \hat{n}_{k}\right| 100\right\rangle=1, \eta_{2}=\left\langle 010\left|\sum_{k=1}^{3} \hat{n}_{k}\right| 010\right\rangle=1$ e $\eta_{4}=\left\langle 001\left|\sum_{k=1}^{3} \hat{n}_{k}\right| 001\right\rangle=1$. Portanto, $\langle\rho(t)\rangle=1 / N \sum_{\mu=1,2,4} \eta_{\mu} P_{\mu}(t)=1 / 3(1 / 3+$ $1 / 3+1 / 3)=1 / 3$, conforme esperado. Também pode-se calcular a densidade média da seguinte maneira: $\langle\rho(t)\rangle=(1 / N) \sum_{\mu}\langle\mu|\hat{n}| P(t)\rangle=(1 / 3)[\langle 100|\hat{n}(|100\rangle+|010\rangle+$ $|001\rangle) / 3+\langle 010|\hat{n}(|010\rangle+|010\rangle+|001\rangle) / 3+\langle 001|\hat{n}(|001\rangle+|010\rangle+|001\rangle) / 3]=1 / 3$.

Derivando a Eq. (4.24) em relação ao tempo e utilizando a Eq. (4.20), tem-se:

$$
\begin{aligned}
\frac{d\langle\rho(t)\rangle}{d t}= & \frac{1}{N} \sum_{\mu} \eta_{\mu} \frac{d P_{\mu}(t)}{d t} \\
& =-\frac{1}{N} \sum_{\mu, \nu} \eta_{\mu} P_{\nu}(t) H_{\mu \nu} \\
& =-\frac{1}{N} \sum_{\mu, \nu} \eta_{\mu} P_{\nu}(t)\langle\mu|\hat{H}| \nu\rangle .
\end{aligned}
$$

De acordo com Eq. (4.25), $\hat{H}$ age sobre as configurações $|\nu\rangle$ do sistema, levando-as às configurações $|\mu\rangle$. Desta forma, para quantificar e entender os resultados gerados pela ação dos operadores contidos no gerador de translações temporais, considere os seguintes exemplos para cada operador da Eq. (4.25):

i) termo de cura da Eq. (4.25): $\sum_{\mu, \nu} \eta_{\mu}\left\langle\mu\left|\sum_{k} \sigma_{k}^{-}\right| \nu\right\rangle$, onde é considerada a configuração $|\nu\rangle=|11100\rangle$. Para esta configuração com um número de infectados $\eta_{\nu}=3$, a ação do operador cura sobre todos os indivíduos infectados gera as seguintes configurações: $\sum_{k} \hat{\sigma}_{k}^{-}|11100\rangle=|11000\rangle+|10100\rangle+|01100\rangle$. Desta forma, a soma conterá 3 termos, um para cada configuração $|\mu\rangle$ possível, de modo que $\sum_{\mu, \nu} \eta_{\mu}\left\langle\mu\left|\sum_{k} \sigma_{k}^{-}\right| 11100\right\rangle=\eta_{\mu_{1}}\left\langle\mu_{1} \mid 11000\right\rangle+\eta_{\mu_{2}}\left\langle\mu_{2} \mid 10100\right\rangle+\eta_{\mu_{3}}\left\langle\mu_{3} \mid 01100\right\rangle$, que só é não-nula quando $\left|\mu_{i}\right\rangle$ é igual às próprias configurações geradas $|11000\rangle,|10100\rangle$ e $|01100\rangle$. Portanto, tem-se um total de 3 possíveis configurações $\left|\mu_{i}\right\rangle$, cada uma delas contendo $\eta_{\mu_{i}}=2$ infectados. De maneira geral, pode-se então escrever que $\sum_{\mu} \eta_{\mu}\left\langle\mu\left|\sum_{k} \sigma_{k}^{-}\right| \nu\right\rangle=\left(\eta_{\nu}-1\right) \eta_{\nu}$.

ii) termo de infecção da Eq. (4.25): $\sum_{\mu} \eta_{\mu}\left\langle\mu\left|\sum_{k} \sigma_{k}^{+}\right| \nu\right\rangle$, onde é considerada a configuração $|\nu\rangle=|11100\rangle$. Para esta configuração com um número de infectados $\eta_{\nu}=3$, a ação do operador de infecção sobre todos os indivíduos suscetíveis gera as seguintes configurações: $\sum_{k} \hat{\sigma}_{k}^{+}|11100\rangle=|11110\rangle+|11101\rangle$. Desta forma, a soma conterá 3 termos, um para cada configuração $|\mu\rangle$ possível, de modo que 
$\sum_{\mu} \eta_{\mu}\left\langle\mu\left|\sum_{k} \sigma_{k}^{+}\right| 11100\right\rangle=\eta_{\mu_{1}}\left\langle\mu_{1} \mid 11110\right\rangle+\eta_{\mu_{2}}\left\langle\mu_{2} \mid 11101\right\rangle$, que só é não-nula quando $\left|\mu_{i}\right\rangle$ é igual às próprias configurações geradas $|11110\rangle$ e $|11101\rangle$. Portanto, tem-se um total de 2 possíveis configurações $\left|\mu_{i}\right\rangle$, cada uma delas contendo $\eta_{\mu_{i}}=4$ infectados. Pode-se generalizar o número de infectados $\eta_{\mu}$ e o número de configurações $|\mu\rangle$ de modo que $\sum_{\mu} \eta_{\mu}\left\langle\mu\left|\sum_{k} \sigma_{k}^{+}\right| \nu\right\rangle=\left(\eta_{\nu}+1\right)\left(N-\eta_{\nu}\right)$.

iii) termo da Eq. (4.25) que contém o operador número $\sum_{\mu} \eta_{\mu}\left\langle\mu\left|\sum_{k} \hat{n}_{k}\right| \nu\right\rangle$, onde é considerada a configuração $|\nu\rangle=|11100\rangle$. Para esta configuração, com número de infectados $\eta_{\nu}=3$, a ação do operador número sobre uma configuração fornece o número total de pessoas infectadas, sem alterar a configuração. Desta forma, a soma sobre todos os indivíduos $k$ será escrita como $\sum_{\mu} \eta_{\mu}\left\langle\mu\left|\sum_{k} \hat{n}_{k}\right| 11100\right\rangle=\eta_{\mu}(\langle\mu|1| 11100\rangle+\langle\mu|1| 11100\rangle+\langle\mu|1| 11100\rangle+\langle\mu|0| 11100\rangle+$ $\langle\mu|0| 11100\rangle)=3 \eta_{\mu}\langle\mu \mid 11100\rangle$, que só é diferente de zero para $|\mu\rangle=|11100\rangle$. Portanto, $\sum_{\mu} \eta_{\mu}\left\langle\mu\left|\sum_{k} \hat{n}_{k}\right| 11100\right\rangle=3 \eta_{\mu}$, onde observa-se que o número de infectados na configuração $|\mu\rangle$ é sempre o mesmo número de infectados na configuração $|\nu\rangle$, devido ao fato do operador número não alterar a configuração sobre a qual age. Desta forma, tem-se o resultado geral: $\sum_{\mu} \eta_{\mu}\left\langle\mu\left|\sum_{k} \hat{n}_{k}\right| \nu\right\rangle=\eta_{\nu}^{2}$.

Com estes resultados, pode-se analisar a ação de cada um dos termos completos contidos no gerador de translações temporais do lado direito da Eq. (4.25):

i) $\alpha / N \sum_{j, k}\left(1-\delta_{j, k}\right)\left(1-\hat{n}_{k}\right) \hat{n}_{j}$.

Considere a ação deste termo sobre a configuração $|\nu\rangle=|1000\rangle$. Na aproximação de campo médio, todos os termos para os quais $j=k$ são nulos. Além disso, o operador $\hat{n}_{j}$ faz com que todos os indivíduos suscetíveis (0) retornem um valor nulo. Assim, tem-se apenas os seguintes termos: $\left(1-\hat{n}_{2}\right) \hat{n}_{1}|1000\rangle+\left(1-\hat{n}_{3}\right) \hat{n}_{1}|1000\rangle+(1-$ $\left.\hat{n}_{4}\right) \hat{n}_{1}|1000\rangle$. Nota-se que a matriz de adjacência e a ação dos operadores número fazem com que sejam não-nulos apenas os termos de interação entre os $\eta_{\nu}$ infectados com os $N-\eta_{\nu}$ suscetíveis, levando a configurações $|\mu\rangle$ com $\eta_{\nu}$ infectados. Com isso, tem-se a generalização do resultado:

$$
\frac{\alpha}{N} \sum_{\mu} \eta_{\mu}\left\langle\mu\left|\sum_{j, k}\left(1-\delta_{j, k}\right)\left(1-\hat{n}_{k}\right) \hat{n}_{j}\right| \nu\right\rangle=\frac{\alpha}{N} \eta_{\nu}^{2}\left(N-\eta_{\nu}\right)
$$

ii) $\gamma \sum_{k} \hat{n}_{k}$.

Sabe-se que $\sum_{\mu} \eta_{\mu}\left\langle\mu\left|\sum_{k} \hat{n}_{k}\right| \nu\right\rangle=\eta_{\nu}^{2}$, pois o operador número retorna o nú- 
mero $\eta_{\nu}$ de infectados e as mesmas configurações com $\eta_{\nu}$ infectados. Portanto,

$$
\gamma \sum_{\mu} \eta_{\mu}\left\langle\mu\left|\sum_{k} \hat{n}_{k}\right| \nu\right\rangle=\gamma \eta_{\nu}^{2}
$$

iii) $-\alpha / N \sum_{j, k}\left(1-\delta_{j, k}\right) \hat{\sigma}_{k}^{+} \hat{n}_{j}$.

Considere a ação deste termo sobre a configuração $|\nu\rangle=|1100\rangle$. Na aproximação de campo médio, todos os termos para os quais $j=k$ são nulos. Além disso, o operador $\hat{n}_{j}$ faz com que todos os indivíduos suscetíveis (0) retornem um valor nulo. Assim, temos apenas os seguintes termos: $\sigma_{3}^{+} \hat{n}_{1}|1100\rangle+\sigma_{4}^{+} \hat{n}_{1}|1100\rangle+$ $\sigma_{3}^{+} \hat{n}_{2}|1100\rangle+\sigma_{4}^{+} \hat{n}_{2}|1100\rangle=2|1110\rangle+2|1101\rangle$. Nota-se que a matriz de adjacência e a ação dos operadores número fazem com que sejam não-nulos apenas os termos de interação entre os $\eta_{\nu}$ infectados com os $N-\eta_{\nu}$ suscetíveis, enquanto que o operador de infecção leva a novas configurações com $\eta_{\nu}+1$ infectados. Com isso, tem-se a generalização do resultado:

$$
-\frac{\alpha}{N} \sum_{\mu} \eta_{\mu}\left\langle\mu\left|\sum_{j, k}\left(1-\delta_{j, k}\right) \hat{\sigma}_{k}^{+} \hat{n}_{j}\right| \nu\right\rangle=-\frac{\alpha}{N}\left(\eta_{\nu}+1\right) \eta_{\nu}\left(N-\eta_{\nu}\right)
$$

iv) $-\gamma \sum_{k} \hat{\sigma}_{k}^{-}$.

Sabe-se que $\sum_{\mu} \eta_{\mu}\left\langle\mu\left|\sum_{k} \sigma_{k}^{-}\right| \nu\right\rangle=\left(\eta_{\nu}-1\right) \eta_{\nu}$, ou seja, o operador de cura gera $\eta_{\nu}$ configurações com $\eta_{\nu}-1$ infectados. Portanto,

$$
-\gamma \sum_{\mu} \eta_{\mu}\left\langle\mu\left|\sum_{k} \hat{\sigma}_{k}^{-}\right| \nu\right\rangle=-\gamma\left(\eta_{\nu}-1\right) \eta_{\nu}
$$

Voltando à Eq. (4.25) e substituindo o termo $\sum_{\mu} \eta_{\mu}\langle\mu|\hat{H}| \nu\rangle$ pelos resultados encontrados nas Eqs. (4.26) à (4.29), tem-se

$$
\begin{aligned}
\frac{d\langle\rho(t)\rangle}{d t} & =-\frac{1}{N} \sum_{\nu}\left[P_{\nu}(t) \frac{\alpha}{N} \eta_{\nu}\left(N-\eta_{\nu}\right)\left(\eta_{\nu}-\eta_{\nu}-1\right)+P_{\nu}(t) \gamma \eta_{\nu}^{2}-P_{\nu}(t) \gamma \eta_{\nu}\left(\eta_{\nu}-1\right)\right] \\
& =\frac{\alpha}{N} \sum_{\nu} \eta_{\nu} P_{\nu}(t)-\frac{\alpha}{N^{2}} \sum_{\nu} \eta_{\nu}^{2} P_{\nu}(t)-\frac{\gamma}{N} \sum_{\nu} \eta_{\nu} P_{\nu}(t) \\
& =\alpha\langle\rho(t)\rangle-\alpha\left\langle\rho^{2}(t)\right\rangle-\gamma\langle\rho(t)\rangle .
\end{aligned}
$$

Sendo a variância dada por $\sigma^{2}(t)=\left\langle\rho^{2}(t)\right\rangle-\langle\rho(t)\rangle^{2}$, então $\left\langle\rho^{2}(t)\right\rangle=\sigma^{2}(t)+\langle\rho(t)\rangle^{2}$. Com esta consideração e definindo o parâmetro $\rho_{e q}=1-\gamma / \alpha$, ao rearranjar os termos da Eq. (4.30), obtém-se:

$$
\frac{d\langle\rho(t)\rangle}{d t}=\alpha\left[\rho_{e q}-\langle\rho(t)\rangle\right]\langle\rho(t)\rangle-\alpha \sigma^{2}(t)
$$




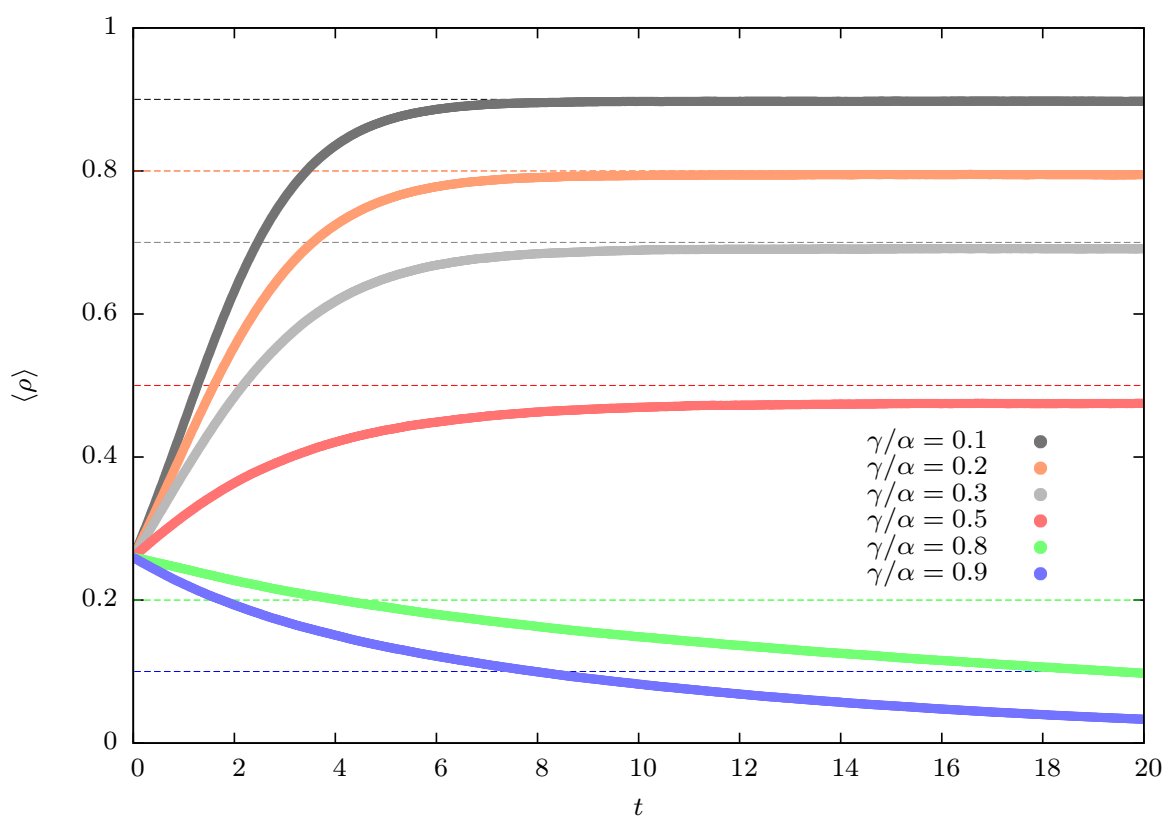

Figura 4.5: Evolução da densidade de infectados como função do tempo (linhas cheias). Para valores de $\gamma / \alpha=0.1,0.2,0.3$, portanto com alta taxa de infecção e baixa taxa de cura, o número de infectados aumenta muito e rapidamente, atingindo os valores $\rho_{e q}$ previstos pela equação compartimental (linhas tracejadas com cores correspondentes aos respectivos valores de $\gamma / \alpha$ ), sendo a variância muito próxima de zero. Conforme a taxa de cura aumenta, o número de infectados diminui para um valor abaixo do valor de equilíbrio. Simulações de Monte Carlo realizadas com $10^{6}$ amostras e para $N=50$.

A equação obtida contém o termo da flutuação na densidade de infectados, que é inerente ao processo e que ocorre devido à natureza discreta do que está sendo medido. Neste caso, imagine que queria-se contar o número médio de indivíduos infectados em uma população. A cada instante, escolhe-se ao acaso um indivíduo e verifica-se se está infectado ou não. Se a população for grande (por exemplo, $N=$ 100.000), a contagem do número de infectados apresenta flutuações tão pequenas que podem ser desprezadas. No entanto, se a população for pequena (por exemplo, $N=50$ ), a contagem do número de infectados apresenta flutuações consideráveis.

A figura 4.5 mostra a evolução temporal do número de infectados de acordo com a Eq. (4.31) para valores de $\gamma / \alpha$ variável e o desvio das soluções dos valores de equilíbrio do modelo compartimental, enquanto que a figura (4.6) mostra evolução temporal do número de infectados de acordo com a Eq. (4.31) para valores de $N$ variável.

Pode-se também obter uma equação para a evolução temporal da variância. 

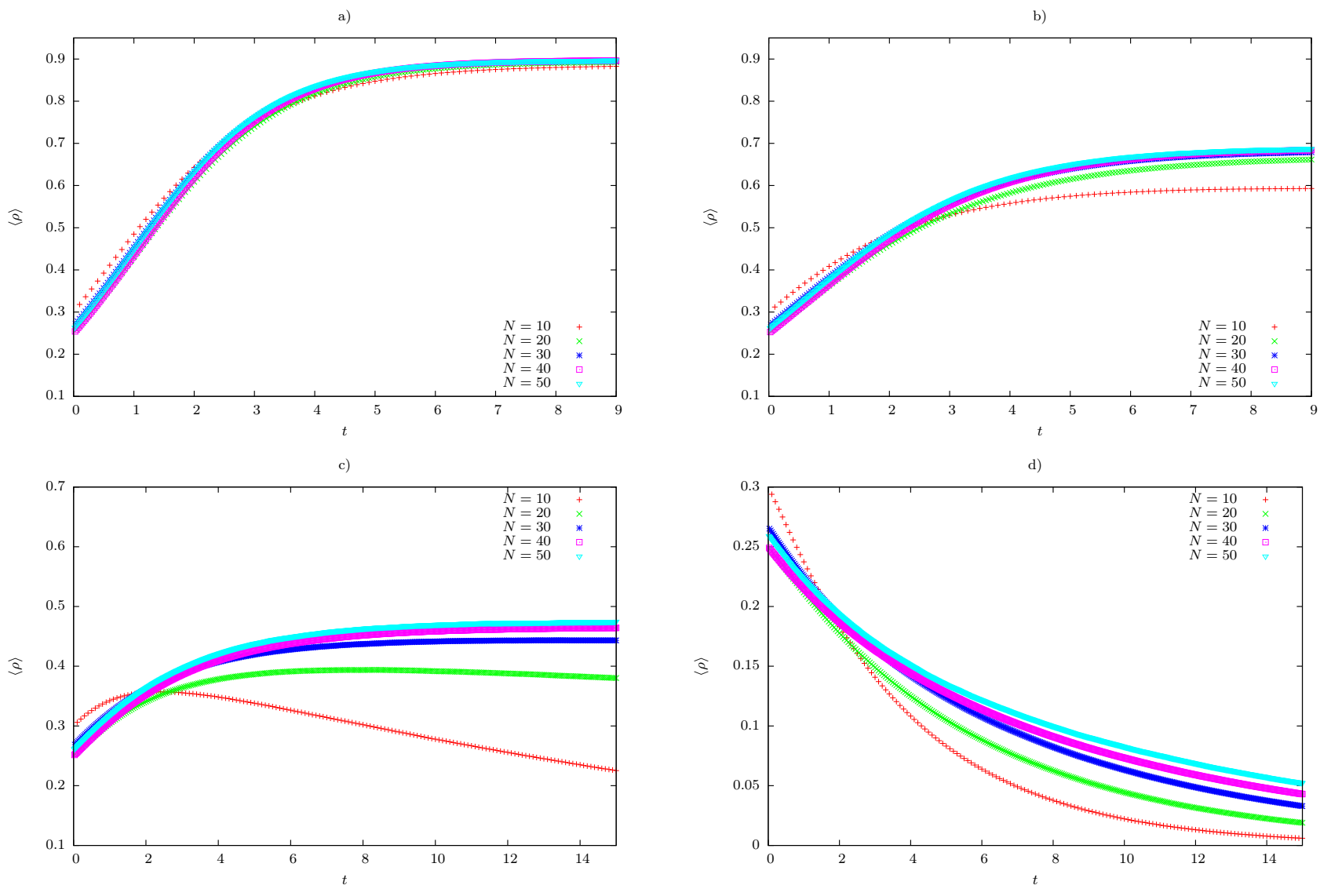

Figura 4.6: Evolução temporal do número de infectados para diferentes valores de $N$ : a) $\gamma / \alpha=$ 0.1 , b) $\gamma / \alpha=0.3$, c) $\gamma / \alpha=0.5$ e d) $\gamma / \alpha=0.9$. Em todos os casos, podemos observar que quanto menores os valores de $N$, mais as flutuações agem diminuindo a densidade de infectados no equilíbrio. No começo da epidemia, no entanto, tem-se o efeito contrário. Nota-se também que os efeitos de flutuação são menores para valores de $\gamma / \alpha$ baixos em relação ao observado para valores de $\gamma / \alpha$ altos, com uma diferença máxima aparente para $\gamma / \alpha=0.5$ e $N=10$. Simulações de Monte Carlo realizadas com $10^{6}$ amostras.

Para tanto, calcula-se a derivada temporal de $\sigma^{2}$, onde $\sigma^{2}=\left\langle\rho^{2}\right\rangle-\langle\rho\rangle^{2}$, obtendo-se:

$$
\frac{d \sigma^{2}}{d t}=\frac{d\left\langle\rho^{2}\right\rangle}{d t}-2\langle\rho\rangle \frac{d\langle\rho\rangle}{d t}
$$

O único termo da equação anterior que não conhecemos é o primeiro termo do lado direito. Para calculá-lo, note que $\left\langle\rho^{2}\right\rangle=\sum_{\mu} \eta_{\mu}^{2} P_{\mu}(t) / N^{2}$, de modo que

$$
\frac{d\left\langle\rho^{2}\right\rangle}{d t}=-\frac{1}{N^{2}} \sum_{\mu} \eta_{\mu}^{2} P_{\nu}(t)\langle\mu|\hat{H}| \nu\rangle,
$$

onde utiliza-se a Eq. (4.20), da mesma maneira que foi feito na derivação da Eq. (4.25). Agora, é necessário, novamente, analisar termo-a-termo a ação de $\hat{H}$ sobre as configurações: 
i) $\sum_{\mu} \eta_{\mu}^{2}\left\langle\mu\left|\sum_{j, k}\left(1-\delta_{j, k}\right)\left(1-\hat{n}_{k}\right) \hat{n}_{j}\right| \nu\right\rangle=\eta_{\nu}^{2} \eta_{\nu}\left(N-\eta_{\nu}\right)$. A matriz de adjacência e a ação dos operadores número fazem com que sejam não-nulos somente os termos de interação entre os $\eta_{\nu}$ infectados com os $N-\eta_{\nu}$ suscetíveis, gerando configurações com $\eta_{\nu}$ infectados.

ii) $\sum_{\mu} \eta_{\mu}^{2}\left\langle\mu\left|\sum_{j, k}\left(1-\delta_{j, k}\right) \hat{\sigma}_{k}^{+} \hat{n}_{j}\right| \nu\right\rangle=\left(\eta_{\nu}+1\right)^{2} \eta_{\nu}\left(N-\eta_{\nu}\right)$. Novamente, a matriz de adjacência e a ação do operador número fazem com que sejam não-nulos os termos de interação entre os $\eta_{\nu}$ infectados com os $N-\eta_{\nu}$ suscetíveis, enquanto que o operador de infecção age gerando configurações com $\eta_{\nu}+1$ infectados.

iii) $\sum_{\mu} \eta_{\mu}^{2}\left\langle\mu\left|\sum_{j, k} \hat{n}_{k}\right| \nu\right\rangle=\eta_{\nu} \eta_{\nu}^{2}$. O operador número age retornando o número $\eta_{\nu}$ de infectados e as mesmas configurações com $\eta_{\nu}$ infectados.

iv) $\sum_{\mu} \eta_{\mu}^{2}\left\langle\mu\left|\sum_{j, k} \hat{\sigma_{k}^{-}}\right| \nu\right\rangle=\eta_{\nu}\left(\eta_{\nu}-1\right)^{2}$. O operador de cura age gerando $\eta_{\nu}$ novas configurações com $\eta_{\nu}-1$ infectados.

Com os resultados acima, pode-se finalmente escrever:

$$
\begin{aligned}
\frac{d\left\langle\rho^{2}\right\rangle}{d t} & =-\frac{1}{N^{2}} \sum_{\nu} P_{\nu}\left[\frac{\alpha}{N} \eta_{\nu}^{3}\left(N-\eta_{\nu}\right)-\frac{\alpha}{N} \eta_{\nu}\left(\eta_{\nu}+1\right)^{2}\left(N-\eta_{\nu}\right)+\gamma \eta_{\nu}^{3}-\gamma \eta_{\nu}\left(\eta_{\nu}-1\right)^{2}\right] \\
& =2 \alpha\left\langle\rho^{2}\right\rangle+\frac{\alpha}{N}\langle\rho\rangle-2 \alpha\left\langle\rho^{3}\right\rangle-\frac{\alpha}{N}\left\langle\rho^{2}\right\rangle-2 \gamma\left\langle\rho^{2}\right\rangle+\frac{\gamma}{N}\langle\rho\rangle .
\end{aligned}
$$

Colocando-se os resultados das Eqs. (4.31) e (4.34) na Eq. (4.32), obtém-se a equação para variância, cuja simulação da evolução temporal está mostrada nas Fig. (4.7):

$$
\frac{d \sigma^{2}(t)}{d t}=2 \alpha \sigma^{2}\left(\rho_{e q}+\langle\rho\rangle\right)+\frac{\alpha}{N}\left(\langle\rho\rangle-\left\langle\rho^{2}\right\rangle\right)+\frac{\gamma}{N}\langle\rho\rangle-2 \alpha \Delta_{3},
$$

onde define-se outra variável para o termo que contém o terceiro momento da densidade e a densidade ao cubo: $\Delta_{3}(t) \equiv\left\langle\rho^{3}(t)\right\rangle-\langle\rho(t)\rangle^{3}$. É possível calcular a variação temporal deste termo, de forma análoga ao que foi feito para $\sigma^{2}$, porém ao fazê-lo, obtem-se outro termo com o quarto momento da densidade de infectados, e assim sucessivamente, de modo que este cálculo não traz resultados satisfatórios.

Esta seção é finalizada com um novo sistema de equações (Eqs. (4.31) e (4.35)) que permite investigar a dinâmica do número de infectados, consideradas as flutuações, e a dinâmica das próprias flutuações. Porém, nota-se que existe ainda a dependência da Eq. (4.35) com o termo que contém o termo $\Delta_{3}$, cuja evolução temporal pode ser calculada, conforme dito anteriormente, mas que gostaríamos de simplificar. 
a)

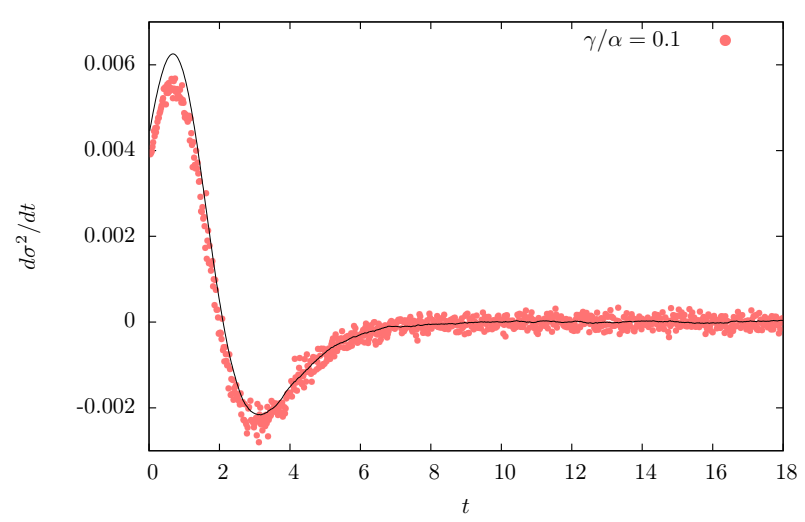

c)

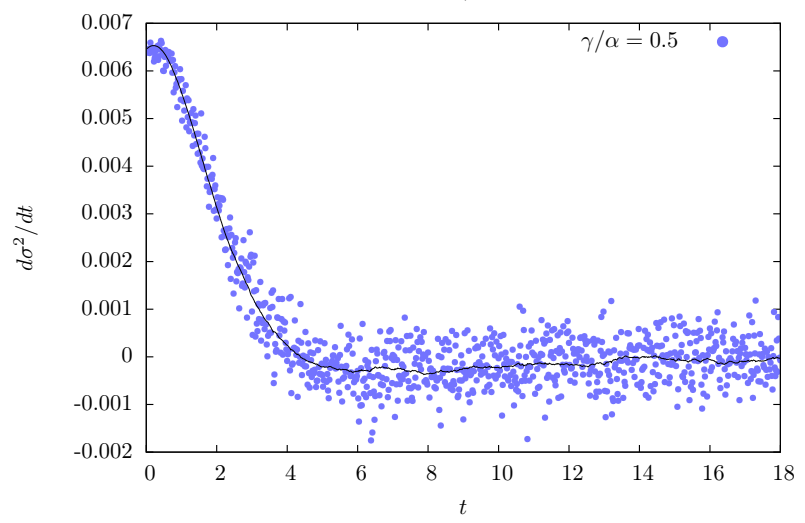

b)

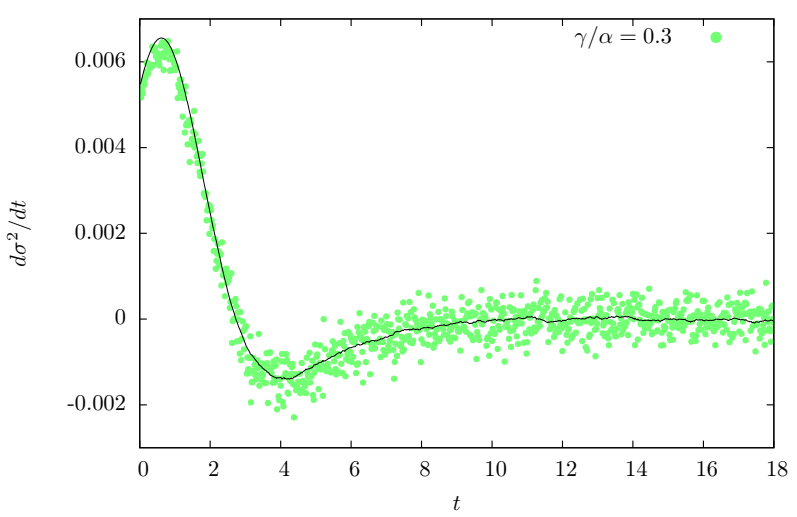

d)

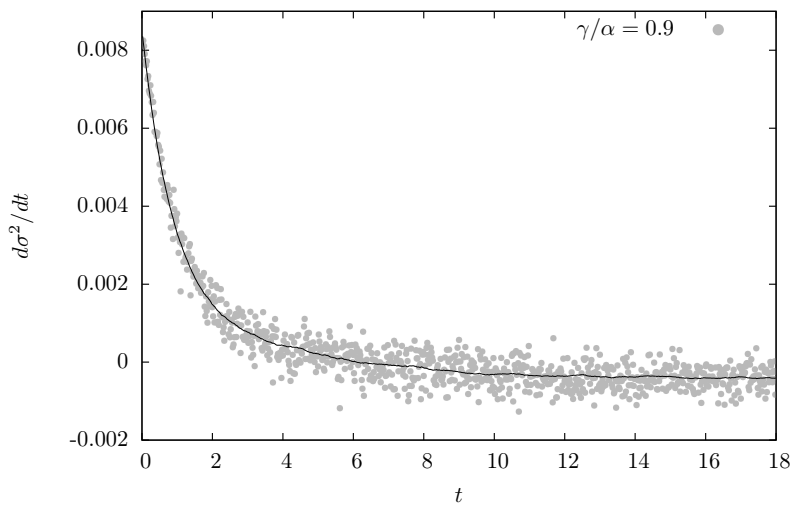

Figura 4.7: Evolução temporal da derivada da variância para diferentes valores de $\gamma / \alpha$ com comportamento esperado no equilíbrio ( $\sigma^{2}$ constante), usando os dados simulados. As linhas cheias representam os valores fornecidos usando a Eq. (4.35). Simulações de Monte Carlo realizadas com $10^{6}$ amostras e para $N=50$.

\subsection{Flutuações Gaussianas}

A busca por uma equação que revele o comportamento ao longo do tempo do termo $\Delta_{3}$ mostra que é possível repetir o procedimento usado para derivar as equações da densidade de infectados e da variância, porém é importante observar que com tal procedimento obtém-se uma expressão que conteria o quarto momento da densidade, e assim sucessivamente. De modo a evitar todos estes cálculos, que não são úteis para esta dissertação, considere que seja possível calcular a variável $\Delta_{3}(t)$ para um determinado limite. Antes disso, no entanto, é importante relacionála com os parâmetros disponíveis. Sendo assim, observe a fórmula do coeficiente de assimetria de uma curva, que mede o quanto uma curva é assimétrica em relação à 
sua média:

$$
\kappa_{3}=\frac{\left\langle x^{3}\right\rangle-\langle x\rangle^{3}-3\langle x\rangle \sigma^{2}}{\sigma^{3}}
$$

sendo $x$ a variável. O termo $\left\langle x^{3}\right\rangle-\langle x\rangle^{3}$ é exatamente o $\Delta_{3}$ que busca-se calcular.

Considerando o limite no qual o tamanho da população é relativamente grande $(N \gg 1)$, dado que as infecções são processos aleatórios é plausível assumir que as flutuações possam ser bem representadas por flutuações Gaussianas para determinados valores de $\gamma / \alpha$ (Figs. 4.8 e 4.9). A vantagem, neste caso, é que sabe-

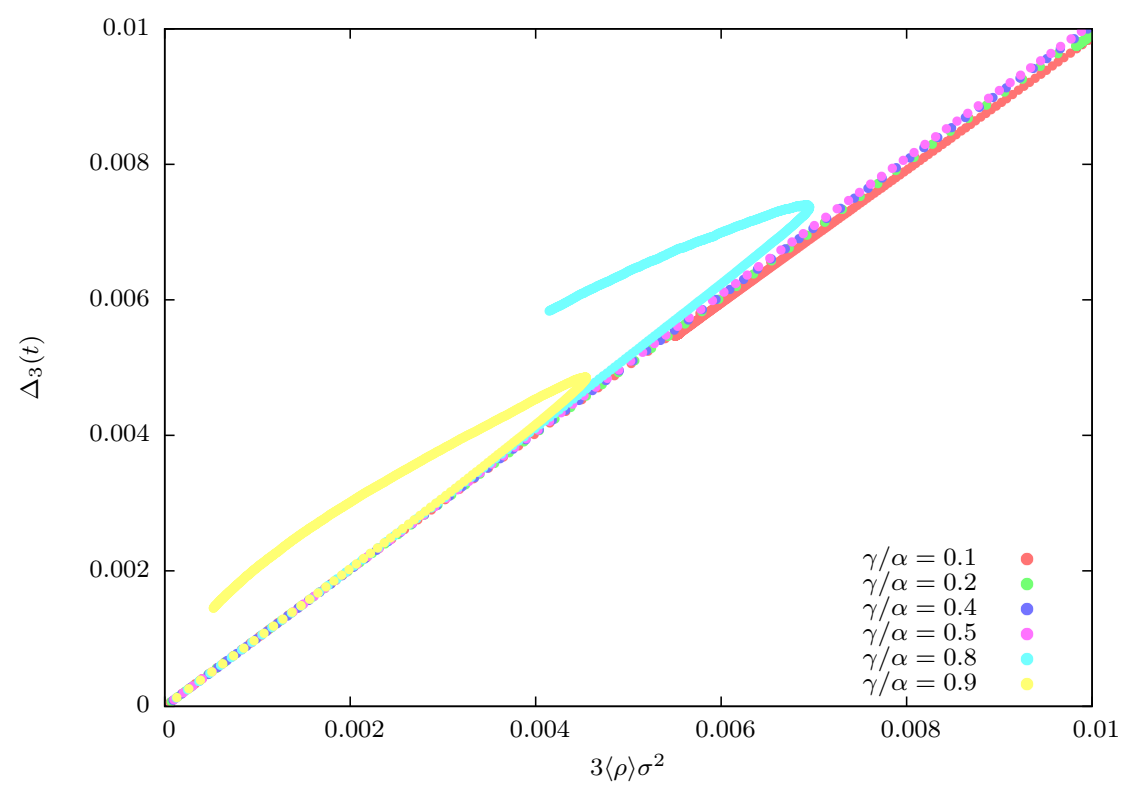

Figura 4.8: $\Delta_{3}(t)$ como função de $\langle\rho\rangle \sigma^{2}$ para valores de $\gamma / \alpha$ variáveis e $N=50$. No limite $N \gg 1$, as flutuações são bem representadas por flutuações Gaussianas, com $\Delta_{3}(t)=3\langle\rho\rangle \sigma^{2}$, apenas para valores de $\gamma / \alpha$ pequenos. Nota-se que, para $\gamma / \alpha=0.8$ e $0.9, \Delta_{3}(t)$ deixa de ser uma função de $\langle\rho\rangle \sigma^{2}$, de modo que, nestes casos, a aproximação Gaussiana falha. Simulação de Monte Carlo realizada com $10^{6}$ amostras.

se que a Gaussiana não possui assimetria em relação à média, ou seja, $\kappa_{3}=0$, o que resulta em $\Delta_{3}=3\langle x\rangle \sigma^{2}$, de acordo com a Eq. (4.36). Deste modo, para flutuações Gaussianas, obtém-se $\Delta_{3}=3\langle\rho(t)\rangle \sigma^{2}(t)$. Colocando este resultado na Eq. (4.35), chega-se a uma equação diferencial para a variância dada por:

$$
\frac{d \sigma^{2}}{d t}=2 \alpha \sigma^{2}\left(\rho_{e q}-2\langle\rho(t)\rangle\right)
$$

onde $N \gg 1$, portanto termos de ordem $1 / N$ são ignorados. Com isso, tem-se um 


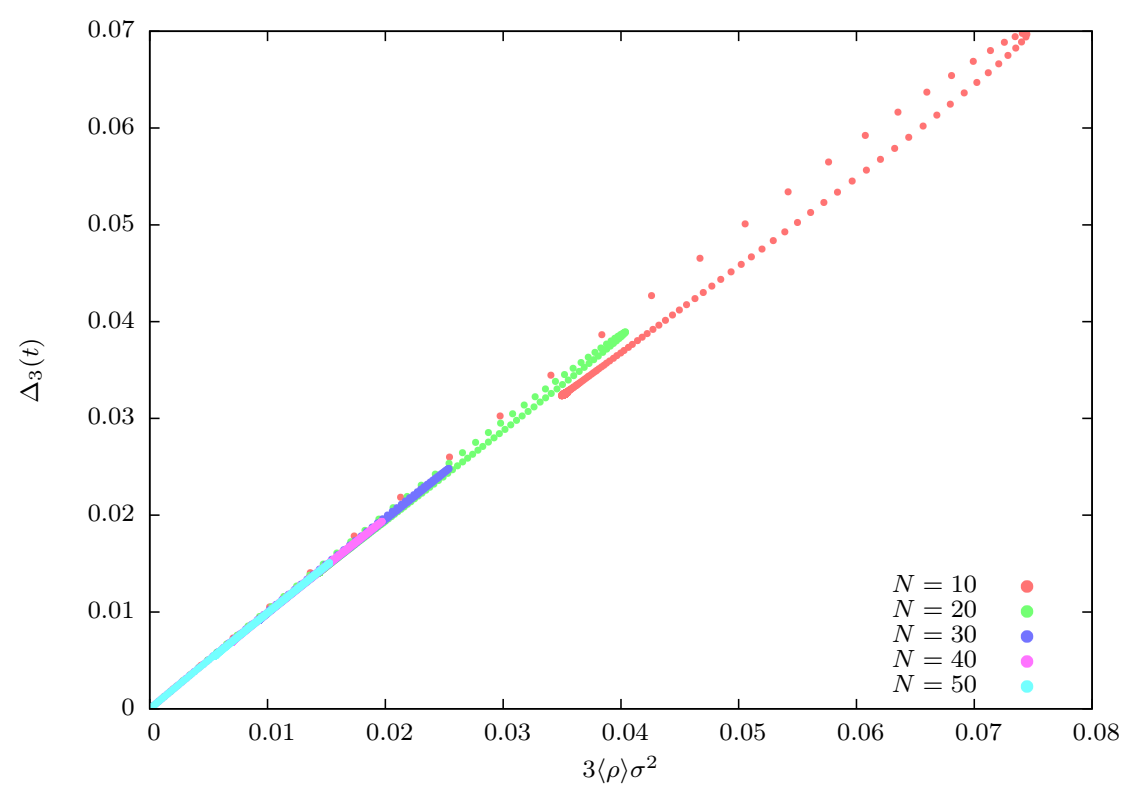

Figura 4.9: $\Delta_{3}(t)$ como função de $\langle\rho\rangle \sigma^{2}$ para valores de $N$ variáveis e com $\gamma / \alpha=0.1$. Pode-se notar a dependência da aproximação Gaussiana com o valor de $N$. Para $N=30$, 40 e 50, obtém-se a reta esperada $\Delta_{3}(t)=3\langle\rho\rangle \sigma^{2}$, porém, para $N=10$ e $20, \Delta_{3}(t)$ deixa de ser uma função de $\langle\rho\rangle \sigma^{2}$, e a aproximação Gaussiana falha.

sistema de equações completo:

$$
\begin{aligned}
\frac{1}{\alpha} \frac{d\langle\rho(t)\rangle}{d t} & =\left[\rho_{e q}-\langle\rho(t)\rangle\right]\langle\rho(t)\rangle-\sigma^{2}(t), \\
\frac{1}{2 \alpha} \frac{d \sigma^{2}}{d t} & =\sigma^{2}\left(\rho_{e q}-2\langle\rho(t)\rangle\right),
\end{aligned}
$$

e com elas podemos analisar a dinâmica da densidade de infectados e das flutuações. Uma maneira alternativa é rearranjar ambas as equações para obter uma equação diferencial de segunda ordem para a densidade de infectados. Ao fazê-lo, obtém-se:

$$
\frac{d^{2}\langle\rho\rangle}{d t^{2}}=3 \alpha\left(\rho_{e q}-\langle\rho\rangle\right)\left[\frac{d\langle\rho\rangle}{d t}-\frac{2 \alpha}{3}\left(\rho_{e q}-\langle\rho\rangle\right)\langle\rho\rangle\right]
$$




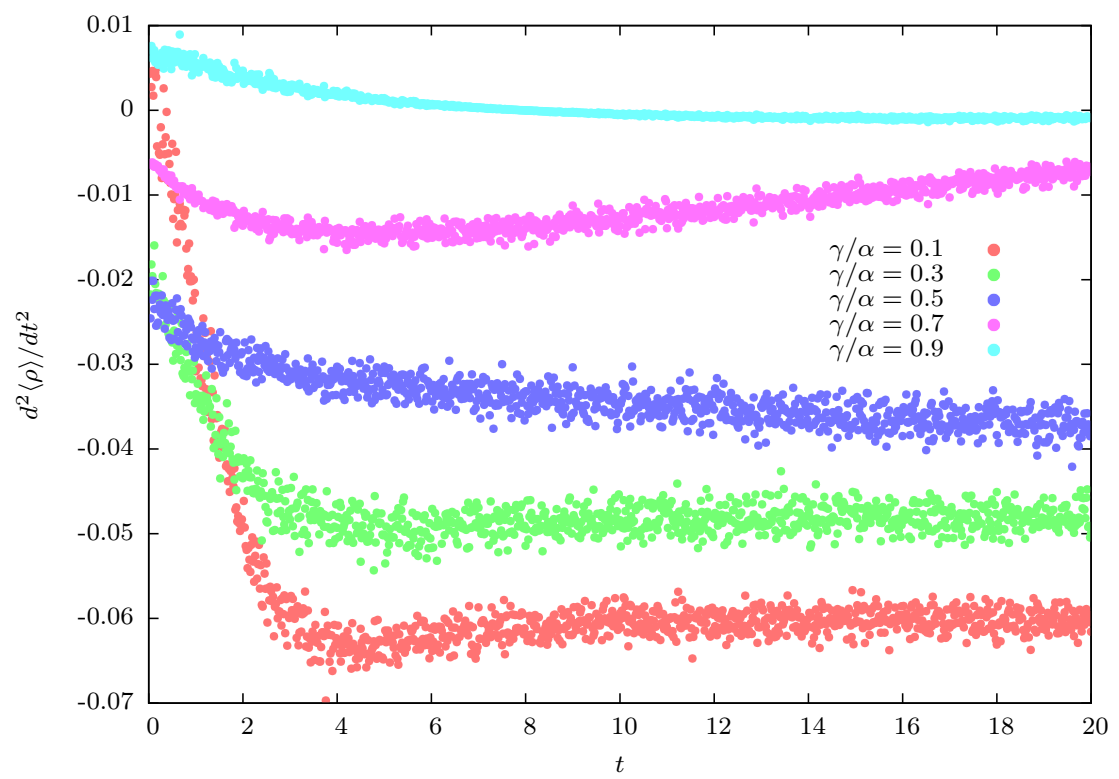

Figura 4.10: Derivada segunda em relação ao tempo da densidade de infectados. Simulação de Monte Carlo realizada utilizando-se a Eq. (4.39), com $10^{6}$ amostras e para $N=50$, com valores de $\gamma / \alpha$ variáveis. 

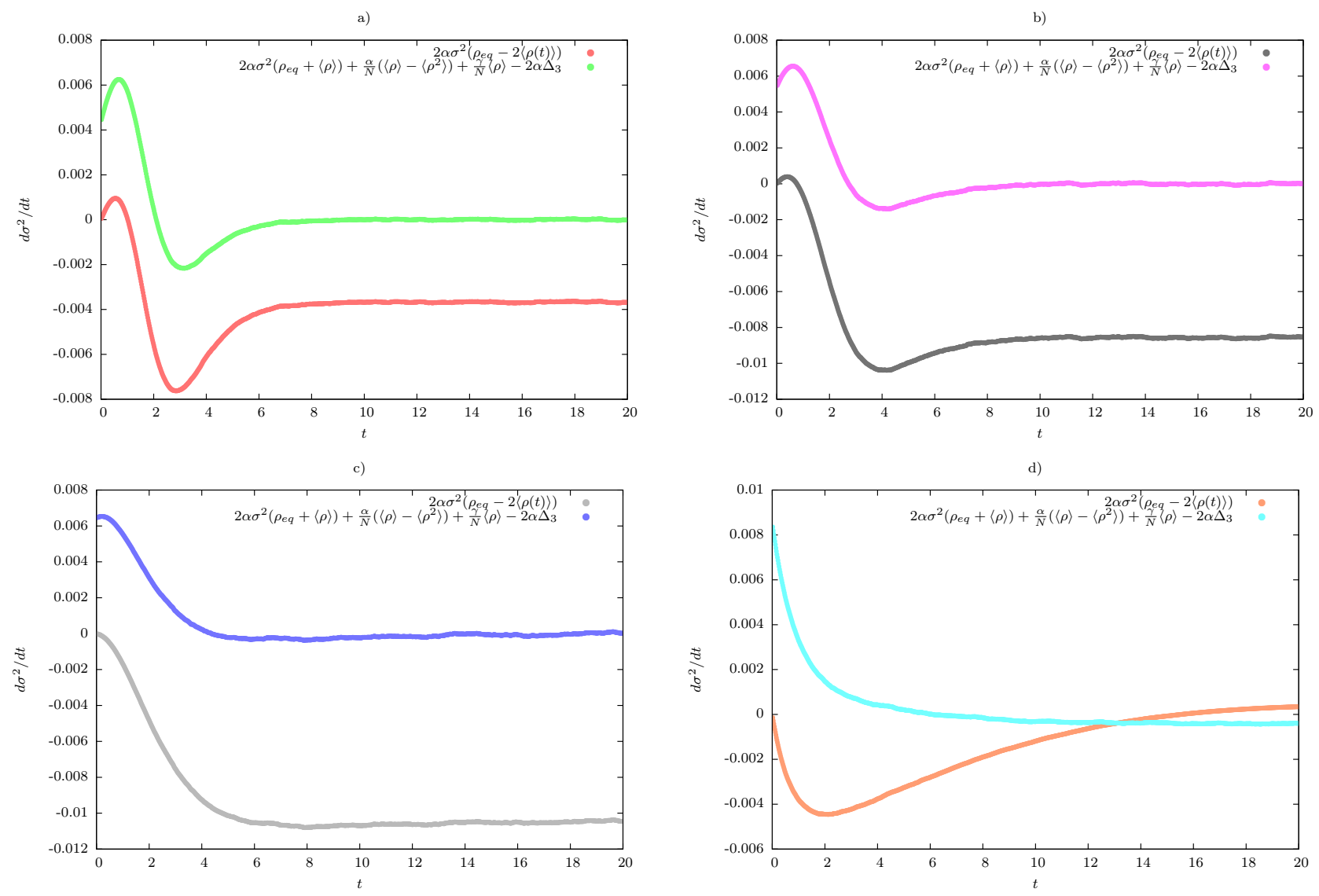

Figura 4.11: Evolução temporal da derivada da variância para as Eqs. (4.35) e (4.37). a) $\gamma / \alpha=0.1$, b) $\gamma / \alpha=0.3$, c) $\gamma / \alpha=0.5$ e d) $\gamma / \alpha=0.9$. Observa-se um comportamento semelhante de $d \sigma^{2} / d t$ tanto para o caso com flutuações Gaussianas (Eq. (4.37)) quanto para o caso geral (Eq. (4.35)) sem aproximações. Simulações de Monte Carlo realizadas com $10^{6}$ amostras e para $N=50$. 


\subsection{Função de correlação}

Considera-se, agora, os casos nos quais $\Delta_{3}(t) \neq 3\langle\rho\rangle \sigma^{2}$, ou seja, casos em que não se pode assumir flutuações Gaussianas. Novamente, é preciso relacionar $\Delta_{3}(t)$ com os parâmetros disponíveis. Para isso, atenta-se à função de autocorrelação temporal da densidade, pelo fato de que funções de correlação e variância possuem mesma magnitude e forma matemática similar:

$$
C_{\rho \rho}(t) \equiv\langle\rho(t+\delta t) \rho(t)\rangle-\langle\rho(t)\rangle^{2}
$$

onde é analisada a correlação entre $\rho(t+\delta t)$ e $\rho(t)$. Na formulação matemática com a qual estamos trabalhando, tem-se que

$$
\langle\rho(t+\delta t) \rho(t)\rangle=\frac{1}{N^{2}} \sum_{\mu} \sum_{j, k}\left\langle\mu\left|\hat{n}_{k} e^{-\hat{H} \delta t} \hat{n}_{j}\right| P(t)\right\rangle,
$$

onde o termo $e^{-\hat{H} \delta t}$ representa a evolução temporal que permite calcular a densidade de infectados em um tempo $t+\delta t$. Aqui, será considerada a expansão da função $e^{-\hat{H} \delta t}$ até primeira ordem apenas, de modo que tem-se:

$$
\langle\rho(t+\delta t) \rho(t)\rangle=\frac{1}{N^{2}} \sum_{\mu, \nu} \sum_{j, k}\left\langle\mu\left|\hat{n}_{k}(-\hat{H} \delta t) \hat{n}_{j} P_{\nu}(t)\right| \nu\right\rangle,
$$

onde foi feita a expansão do vetor probabilidade na base das configurações.

$\hat{H}$ assume a forma dada pela Eq. (4.21) e age sobre as configurações $|\nu\rangle$, juntamente com os operadores número. Assim sendo, de maneira análoga ao que foi feito para o cálculo de $d\langle\rho\rangle / d t$ e $d \sigma^{2} / d t$, calcula-se o termo $\sum_{\mu, \nu} \sum_{j, k}\left\langle\mu\left|\hat{n}_{k}(-\hat{H} \delta t) \hat{n}_{j} P_{\nu}(t)\right| \nu\right\rangle$ da Eq. (4.42) através da análise da ação de todos os operadores, que colocado na Eq. (4.40) resulta em:

$$
C_{\rho \rho}(t)=\alpha \delta t\left[\rho_{e q}\left(\langle\rho\rangle^{2}-\sigma^{2}\right)-\left\langle\rho^{3}\right\rangle\right]+\sigma^{2}
$$

Observa-se, porém, que $C_{\rho \rho}(t)$ não exibe uma forma funcional simples, como a encontrada no caso de flutuações Gaussianas, que possa ser usada para substituir $\Delta_{3}$ na Eq. (4.35), pois apresenta uma dependência explícita do tempo. Considera-se, então, uma função de autocorrelação normalizada dada por $D_{\rho \rho} \equiv\left(C_{\rho \rho}-\sigma^{2}\right) / \delta t\langle\rho\rangle^{2}$, cuja forma final pode ser escrita como

$$
D_{\rho \rho}=\alpha\left(\rho_{e q}-\frac{\left\langle\rho^{3}\right\rangle}{\langle\rho\rangle^{2}}\right)+\alpha \rho_{e q} \frac{\sigma^{2}}{\langle\rho\rangle^{2}} .
$$




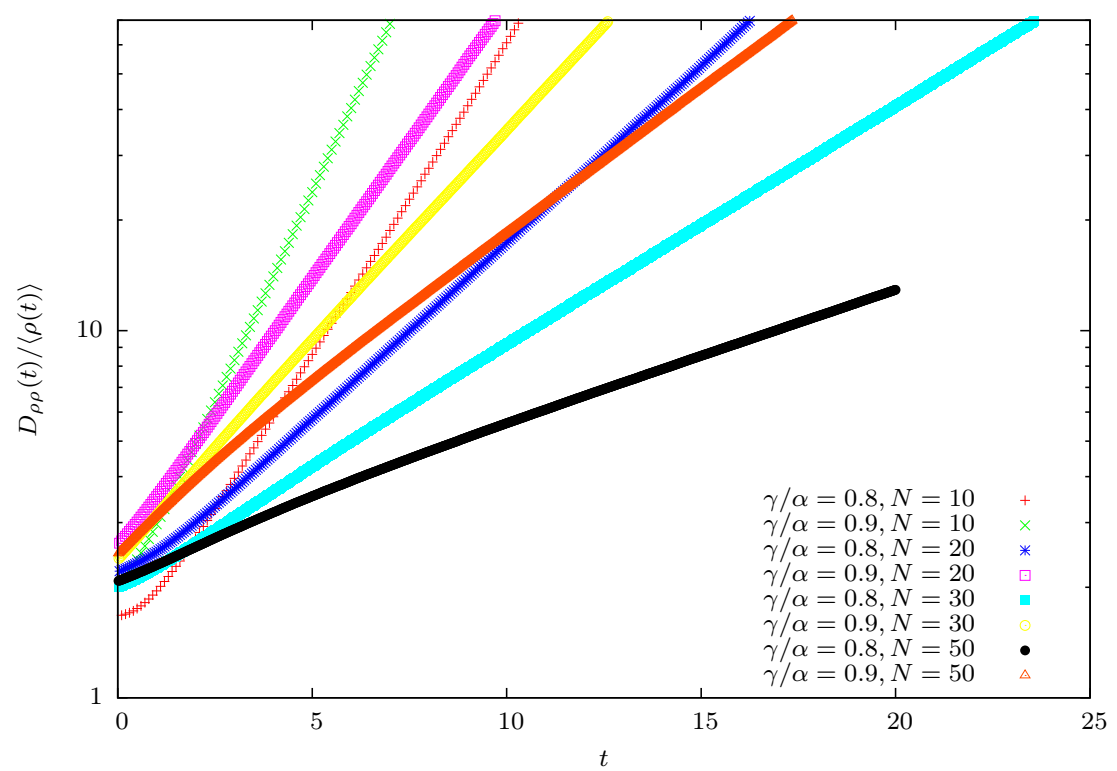

Figura 4.12: Evolução temporal de $D_{\rho \rho}(t) /\langle\rho(t)\rangle$ para valores de $N$ variáveis e $\gamma / \alpha=0.8$ e 0.9 . Para estes valores de $\gamma / \alpha$ e $N=10,20$, a consideração de flutuações Gaussianas falha. Nestes casos, a função de autocorrelação normalizada pode ser usada para substituir o termo $\Delta_{3}$, com $D_{\rho \rho}(t) /\langle\rho(t)\rangle$ apresentando um crescimento exponencial. Simulações de Monte Carlo realizadas $\operatorname{com} 10^{6}$.

Observa-se que esta função de autocorrelação normalizada apresenta a seguinte propriedade: no limite em que $N \rightarrow \infty$, quando o tamanho da população é muito grande e as flutuações podem ser consideradas desprezíveis $\left(\sigma^{2} \rightarrow 0\right)$, ela recupera a seguinte equação

$$
D_{\rho \rho}=\alpha\left[\rho_{e q}-\langle\rho(t)\rangle\right]
$$

que nada mais é do que a equação compartimental (4.5) dividida por $\rho: d \ln \rho / d t=$ $\alpha\left(\rho_{e q}-\rho\right)$. A vantagem neste caso é que se compararmos esta última equação com a Eq. (4.45), torna-se possível inferir a dependência temporal de $D_{\rho \rho}$, observando que:

$$
D_{\rho \rho} \propto D_{0} e^{t / \tau}\langle\rho(t)\rangle
$$

onde $D_{0}$ e $\tau$ são parâmetros de ajuste.

\subsection{Conclusões do capítulo}

Ao descrever o modelo epidêmico SIS de maneira análoga a um sistema de spin 1/2, encontra-se um gerador de translações temporais dado pela Eq. (4.21), 
que descreve a evolução temporal do vetor probabilidade.

Com interesse em analisar como se comporta a evolução da densidade de infectados para uma população pequena, na qual é natural supor que haja flutuações no número de infectados, encontra-se que a ação do gerador de translações temporais sobre as configurações gera a Eq. (4.31), análoga à equação compartimental (4.5), mas que contém o termo $\sigma^{2}(t)$ das flutuações.

Através das simulações numéricas, verifica-se que, de fato, as flutuações têm efeito considerável quanto menor o valor de $N$ (Fig. 4.6), agindo de modo a diminuir a densidade de infectados no equilíbrio e tornando-a maior no começo do espalhamento da doença. Verifica-se também a dependência das flutuações com a razão $\gamma / \alpha$ (Fig. 4.5), sendo o efeito delas mais pronunciado quanto maior o valor dessa razão.

É possível encontrar também uma equação para a evolução temporal das flutuações, dada pela Eq. (4.35), que contém, no entanto, o termo $\Delta_{3}$. Este termo pode ser tratado de duas maneiras: considerando-se flutuações Gaussianas, $\Delta_{3}=$ $3\langle\rho(t)\rangle \sigma^{2}(t)$, e nesta aproximação pode-se gerar um sistema de equações e uma equação de segundo grau para $\langle\rho(t)\rangle$ (Eq. (4.39)). No entanto, a aproximação de flutuações simétricas falha para valores de $N$ menores e para valores de $\gamma / \alpha$ maiores (Figs. 4.8 e 4.9), tornando-se necessário considerar casos mais gerais de flutuações. Neste ponto, a função de autocorrelação normalizada é usada, e com ela também é possível chegar a um sistema de equações fechado para $\langle\rho(t)\rangle$ e $\sigma^{2}(t)$. Verifica-se, ainda, que $D_{\rho \rho}$ possui crescimento exponencial para $N=10,20$ e $\gamma / \alpha=0.9$, que são valores para os quais falha a aproximação Gaussiana. 


\section{Estados Coerentes}

Em mecânica quântica, observa-se que as partículas possuem uma propriedade fundamental, o spin, que pode ser visto como um momento angular intrínseco. No caso mais simples, no qual o número quântico de spin é $s=1 / 2$, a projeção do spin em uma determinada direção (por exemplo, $z$ ) possui os valores $\hat{S}_{z}\left|s, m_{s}\right\rangle= \pm m_{s} \hbar\left|s, m_{s}\right\rangle= \pm \frac{1}{2} \hbar\left|s, m_{s}\right\rangle$, onde $m_{s}=-s,-s+1, \ldots, s-1, s$ e $\hat{S}_{z}$ é o operador de spin na direção $z$. Considerando-se um certo número de spins 1/2 em uma rede podendo interagir uns com os outros, como é o caso de materias ferromagnéticos (nos quais spins tendem a se alinhar na mesma direção) e antiferromagnéticos (nos quais spins vizinhos alinham-se em direções opostas), o Hamiltoniano do sistema pode ser escrito como:

$$
\hat{H}=\frac{1}{2} \sum_{n \neq m} J_{n m} \hat{S}_{n} \cdot \hat{S}_{m}
$$

que é o modelo de Heinsenberg [47], no qual $n$ e $m$ referem-se aos sítios na rede, $J_{n m}$ são constantes de acoplamento, $\hat{S}_{n}$ são os operadores de spin satisfazendo as relações de comutação $\left[\hat{S}_{n}^{i}, \hat{S}_{n}^{j}\right]=\imath \sum_{k} \epsilon_{i, j, k} S_{n}^{k}$, sendo $\epsilon_{i, j, k}$ o símbolo de Levi-Civita com $i, j, k=x, y, z$. Spins em diferentes sítios comutam.

Tem-se, portanto, um Hamiltoniano escrito em função de operadores de spin, cuja comutação é ela mesma um operador. Analiticamente, trabalhar com operadores é mais complicado do que trabalhar com números. Tendo em vista estas dificuldades, busca-se representações dos operadores de spin como função de outros operadores cuja comutação ou anticomutação é um número complexo, como os operadores canônicos bosônicos ou fermiônicos.

Considere o caso no qual o estado fundamental de uma rede de spins ocorre 


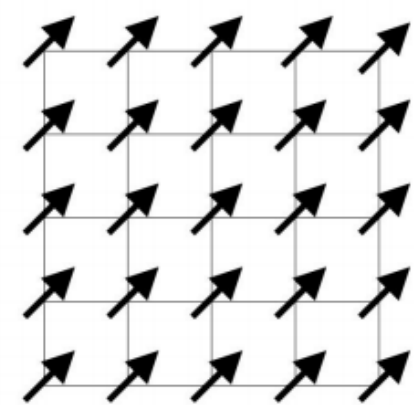

a)

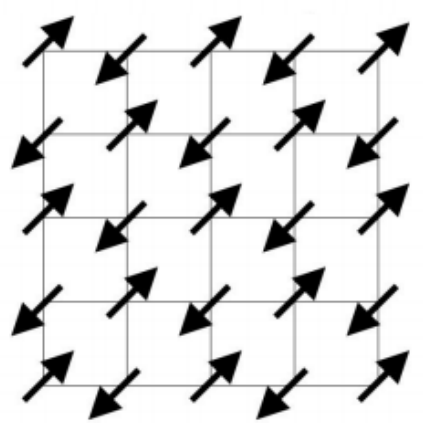

b)

Figura 5.1: a) Spins em uma rede quadrada de um material ferromagnético alinham-se no mesmo sentido, com constante de acoplamento $J<0$ e b) spins em um material antiferromagnético alinham-se em sentidos opostos aos dos vizinhos mais próximos, com constante de acoplamento $J>0$.

quando estão todos alinhados, ou seja, $S_{z}=|S|$ é um máximo, sendo $\hat{S}$ o operador de spin total. É natural esperar que haja pequenas oscilações coletivas dos spins em torno do valor máximo de $S_{z}$, diminuindo ou aumentando ligeiramente este valor, o que é chamado de ondas de spin. Em seus estudos sobre este fenômeno, T. Holstein and H. Primakoff [48] identificaram simetrias contínuas (bosônicas) e propuseram uma transformação que mapeia operadores de spin em operadores de criação e aniquilação bosônicos:

$$
\begin{aligned}
\hat{S}_{z} & =\hat{n}-\hat{S} \\
\hat{S}^{+} & =\hat{a}^{\dagger} \sqrt{2 \hat{S}-\hat{n}} \\
\hat{S}^{-} & =\sqrt{2 \hat{S}-\hat{n}} \hat{a}
\end{aligned}
$$

(ver Apêndice 1 para a derivação completa da transformação). Esta transformação de Holstein-Primakoff (transformação HP), que de maneira simplificada constrói o fato que operadores de spin em sítios diferentes comutam, permite escrever o Hamiltoniano do modelo de Heisenberg (Eq. (5.1)) como função dos operadores bosônicos, simplificando o problema. É importante notar, porém, que a hipótese de pequenas oscilações só é satisfeita se o valor de $S_{z}$ não varia muito em torno do valor máximo de spin (pequenas oscilações), o que, de acordo com a Eq. (5.2a) ocorre para um valor médio de $\hat{n}$ muito menor que $|S|$. Com esta consideração, a expansão das Eqs. (5.2b) e (5.2c) pode ser realizada para $\hat{n} / \hat{S} \ll 1$, já que não é possível trabalhar diretamente com elas na forma apresentada, por conta da raiz quadrada. 
De maneira análoga, também gostaríamos de explorar uma forma de simplificar o gerador de translação temporal não-hermitiano do modelo SIS (Eq. (4.23)), $\mathcal{H}=-\frac{\alpha}{N}\left(\hat{n}+\hat{S}^{+}\right) \hat{n}-\gamma \hat{S}^{-}+(\alpha+\gamma) \hat{n}$, e notamos a semelhança entre o problema de Holstein e Primakoff e o nosso, buscando, assim, proceder utilizando a expansão feita por eles. Uma vez aplicada a transformação HP ao gerador de translações temporais do modelo SIS, escrevendo-o em função dos operadores bosônicos, uma base natural para o problema se torna a base dos estados coerentes. Ao utilizar estes estados, pode-se aproveitar toda a formulação matemática e os conceitos físicos desenvolvidos no contexto da óptica quântica para analisar o problema, considerado o limite em que o número de pessoas infectadas é bem menor do que o número total $N$, como um análogo à aproximação de pequenas oscilações.

Os estados coerentes tornam-se então objeto de nossos estudos e serão inseridos na modelagem matemática do modelo SIS. No entanto, em contraste com o capítulo anterior onde a incerteza na densidade de infectados gera flutuações consideráveis em uma população pequena, neste capítulo, ao usar os estados coerentes como base, faz-se com que as flutuações sejam mínimas em uma população grande.

\subsection{Oscilador harmônico quântico}

Para que se possa introduzir a técnica utilizada neste capítulo, faz-se necessário falar de um sistema-modelo muito importante na Mecânica Quântica, o oscilador harmônico quântico [49], um dos poucos sistemas quânticos que possui uma solução analítica exata. Construído como o análogo do oscilador harmônico clássico, no qual tem-se um sistema massa-mola que obedece às Leis de Newton, estando a massa sujeita à uma força restauradora proporcional a um deslocamento e que gera um potencial do tipo $k x^{2}, k$ sendo uma constante, o oscilador harmônico quântico descreve uma partícula sujeita a um potencial da mesma forma, porém obedecendo à Equação de Schrödinger independente do tempo $\hat{H}|\Psi\rangle=E|\Psi\rangle$.

O Hamiltoniano que descreve o oscilador harmônico quântico para uma partícula de massa $m$ é dado por

$$
\hat{H}_{O H Q}=\frac{\hat{p}^{2}}{2 m}+\frac{k \hat{x}^{2}}{2}=\frac{1}{2 m}\left[\hat{p}^{2}+(m \omega \hat{x})^{2}\right],
$$

onde $\hat{p}$ é o operador momento da partícula, $\hat{x}$ o operador posição, tal que $[\hat{x}, \hat{p}]=i \hbar$, 


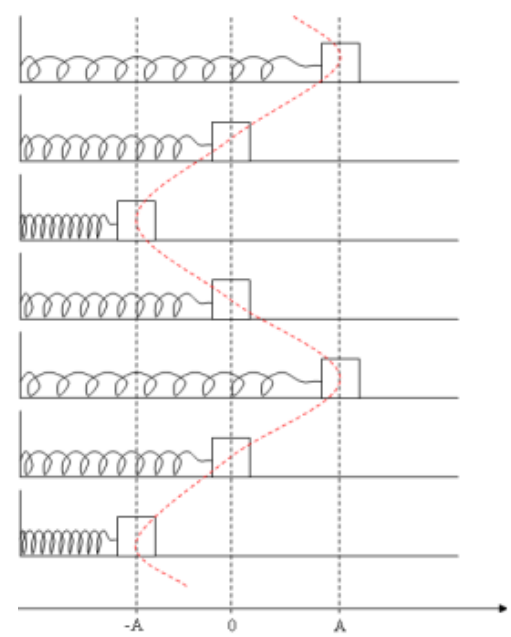

a)

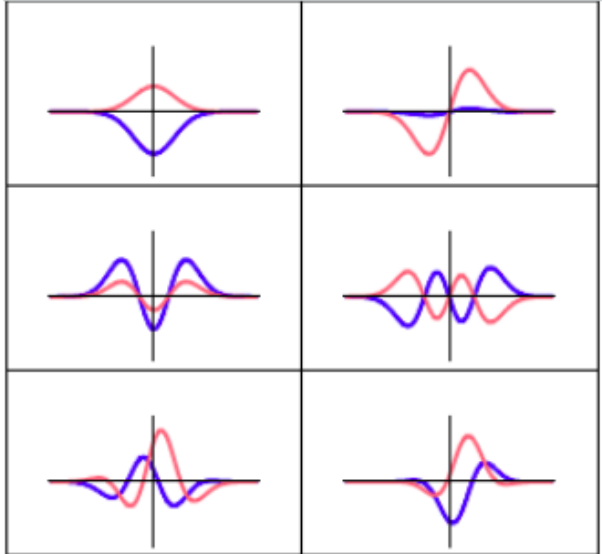

b)

Figura 5.2: a) Sistema massa-mola com a posição da massa dada pela linha tracejada em vermelho e b) soluções da Equação de Schrödinger para o oscilador harmônico quântico, no qual o eixo horizontal representa a posição da função de onda, e o eixo vertical, as partes real (em azul) e imaginária (em vermelho) da função de onda. Fonte: Wikipedia.

e $\omega=\sqrt{k / m}$ é a frequência angular.

Fatorizando a Eq. (5.3), pode-se definir os seguintes operadores:

$$
\begin{aligned}
\hat{a}^{\dagger} & \equiv \frac{1}{\sqrt{2 \hbar m \omega}}(m \omega \hat{x}-i \hat{p}), \\
\hat{a} & \equiv \frac{1}{\sqrt{2 \hbar m \omega}}(m \omega \hat{x}+i \hat{p}),
\end{aligned}
$$

cuja relação de comutação pode ser calculada a partir de suas definições, o que nos fornece

$$
\begin{aligned}
& {\left[\hat{a}, \hat{a}^{\dagger}\right]=1,} \\
& {\left[\hat{a}^{\dagger}, \hat{a}\right]=-1 .}
\end{aligned}
$$

Da multiplicação das Eqs. (5.4) e (5.5), tem-se

$$
\hat{a}^{\dagger} \hat{a}=\frac{1}{2 \hbar m \omega}(m \omega \hat{x}-i \hat{p})(m \omega \hat{x}+i \hat{p})=\frac{\hat{H}_{O H Q}}{\hbar \omega}-\frac{1}{2},
$$

de modo que podemos escrever $\hat{H}_{O H Q}=h \omega\left(\hat{a}^{\dagger} \hat{a}+1 / 2\right)$. Com isto, tem-se o Hamiltoniano do oscilador harmônico quântico escrito em função dos operadores acima definidos.

Define-se também o operador número $\hat{n}=\hat{a}^{\dagger} \hat{a}$, de modo que o Hamiltoniano pode ser finalmente escrito da seguinte forma

$$
\hat{H}_{O H Q}=h \omega(\hat{n}+1 / 2),
$$


sendo que as relações de comutação entre $\hat{n}, \hat{a}^{\dagger}$ e $\hat{a}$ podem ser calculadas com o auxílio das Eqs. (5.6):

$$
\begin{aligned}
{\left[\hat{n}, \hat{a}^{\dagger}\right] } & =\hat{a}^{\dagger}, \\
{[\hat{n}, \hat{a}] } & =-\hat{a} .
\end{aligned}
$$

Note que, dada a Equação de Schrödinger $\hat{H}|\Psi\rangle=E|\Psi\rangle$, a aplicação do operador $\hat{a}^{\dagger}(\hat{a})$ ao estado $|\Psi\rangle$ tem como efeito aumentar (diminuir) a energia de $\hbar \omega$. Por este motivo, dá-se a esses operadores o nome de operadores de criação $\left(\hat{a}^{\dagger}\right)$ e aniquilação $(\hat{a})$.

Considerando, agora, a base de estados $|n\rangle$ do oscilador, que é uma base completa $\sum_{n}|n\rangle\langle n|=\hat{1}$ e ortonormal $\langle n \mid m\rangle=\delta_{n, m}$, tem-se as seguintes relações

$$
\begin{aligned}
\hat{a}^{\dagger}|n\rangle & =\sqrt{n+1}|n+1\rangle, \\
\hat{a}|n\rangle & =\sqrt{n}|n-1\rangle .
\end{aligned}
$$

Portanto, os operadores elevam ou diminuem o estado $n$ do oscilador, como se estivéssemos percorrendo uma escada de estados. Por esse motivo, também são chamados de operadores escada. Além disso, como a matemática dos operadores de criação e aniquilação de bósons é a mesma dos operadores escada (formalismo de segunda quantização, onde os operadores criação e aniquilação removem e criam uma partícula, respectivamente, em um sistema de muitos corpos), eles também são chamados de operadores bosônicos.

Temos, assim, uma solução para o oscilador harmônico quântico em função de operadores que agem aumentando ou diminuindo de um quantum de unidade a energia.

\subsection{Estados coerentes: definição e propriedades}

Os estados coerentes foram introduzidos pela primeira vez em 1926 por Schroedinger para descrever soluções do oscilador harmônico quântico que fossem o mais clássicas possíveis, que são pacotes de onda que não espalham durante sua evolução temporal. Porém, foi apenas em 1963 que Roy J. Glauber, em seu artigo intitulado "Coherent and Incoherent States of the Radiation Field"[50], nomeia os estados inventados por Schrödinger e faz um estudo sobre eles, mostrando que são autoestados 


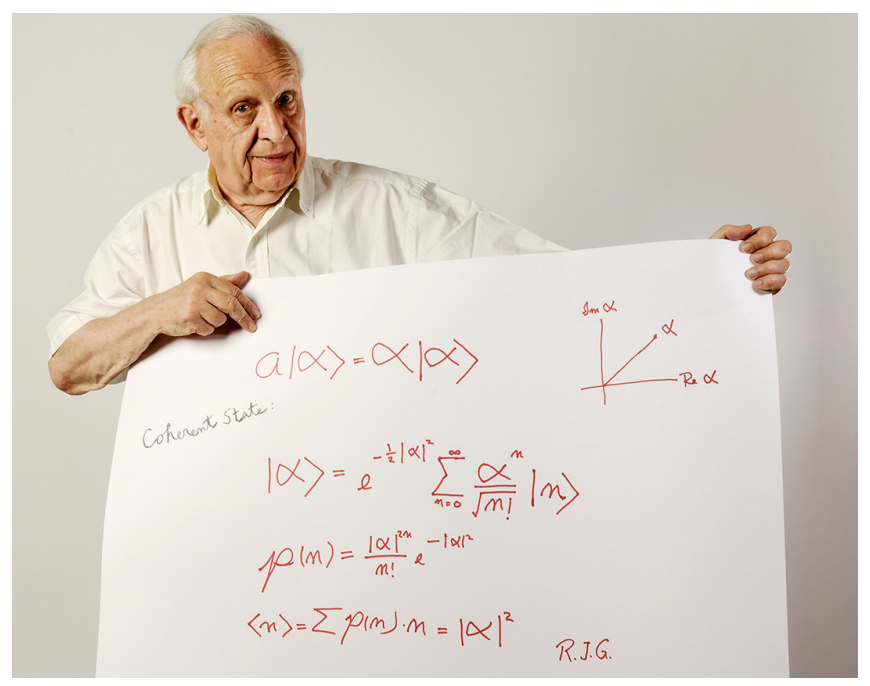

Figura 5.3: Uma homenagem ao físico Roy Jay Glauber (1925-2018) com seus estados coerentes.

do operador aniquilação. Segundo Glauber, fenômenos eletromagnéticos de caráter quântico são muitas vezes descritos por processos nos quais observa-se o aumento ou a diminuição no número de fótons. Este aumento e diminuição é algo que nos remete aos estados do oscilador harmônico quântico anteriormente descrito, onde o Hamiltoniano do problema pode ser escrito em termos de operadores especiais cujas ações consistiam em aumentar ou diminuir de um quantum de unidade a energia, percorrendo, assim, os diferentes estados de energia. Glauber, então, usa o oscilador harmônico como forma de introduzir os estados coerentes. Porém, neste caso, os operadores $\hat{a}^{\dagger}$ e $\hat{a}$ destroem e criam partículas.

Matematicamente, os estados coerentes são definidos como:

$$
\begin{array}{r}
\hat{a}|\lambda\rangle=\lambda|\lambda\rangle, \\
\langle\lambda| \hat{a}^{\dagger}=\lambda^{*}\langle\lambda|,
\end{array}
$$

sendo $\lambda$, em geral, um número complexo, cujo complexo conjugado é dado por $\lambda^{*}$, que pode ser expresso por $\lambda=r e^{i \theta}$, onde $r \in \mathbb{R}$ é a amplitude e $0 \leq \theta \leq 2 \pi$, a fase. Fisicamente, podemos dizer que os estados coerentes permanecem inalterados pela aniquilação de uma partícula ou de uma excitação do campo.

Pode-se encontrar a forma dos estados coerentes por meio de uma expansão em termos da base de estados $|n\rangle$ acima definida, escrevendo-os como uma super- 
posição de infinitos estados quânticos:

$$
|\lambda\rangle=\sum_{n} c_{n}|n\rangle
$$

As a Eqs. (5.14) à Eq. (5.12) quando aplicadas à Eq. (5.11) fornecem a seguinte relação

$$
\hat{a}|\lambda\rangle=\hat{a} \sum_{n} c_{n}|n\rangle=\sum_{n} c_{n} \sqrt{n}|n-1\rangle .
$$

Rearranjando os termos da equação anterior e considerando a normalização $\sum_{n=0}^{\infty}\left|c_{n}\right|^{2}=1$, obtemos

$$
|\lambda\rangle=e^{-|\lambda|^{2} / 2} \sum_{n} \frac{\lambda^{n}}{\sqrt{n !}}|n\rangle .
$$

Se considerarmos o número de ocupacão média de um estado $n$

$$
|\langle n \mid \lambda\rangle|^{2}=\frac{|\lambda|^{2 n}}{n !} e^{-|\lambda|^{2}}
$$

vemos que ele é descrito por uma distribuição de Poisson na variável $\lambda$. Além disso, este resultado pode ser visto, em um contexto de experimentos realizados com laser, como a probabilidade de se detectar $n$ fótons.

Outra propriedade de grande importância desses estados consiste no fato de que eles são tais que sua relação de incerteza é mínima $\Delta x \Delta p=\frac{\hbar^{2}}{2}, \operatorname{com} \Delta x=$ $\Delta p=\frac{\hbar}{\sqrt{2}}$, sendo $\Delta x$ e $\Delta p$ as variâncias na posição e no momento, respectivamente.

Os estados coerentes também formam uma base completa, isto é,

$$
\frac{1}{\pi} \int|\lambda\rangle\langle\lambda| d^{2} \lambda=\hat{1}
$$

onde $d^{2} \lambda=d(\operatorname{Re}[\lambda]) d(\operatorname{Im}[\lambda])$. Para provar a Eq. (5.18), realiza-se a integral em coordenadas polares:

$$
\begin{aligned}
\frac{1}{\pi} \int|\lambda\rangle\langle\lambda| d^{2} \lambda & =\frac{1}{\pi} \int d^{2} \lambda e^{|\lambda|^{2}} \sum_{n, m=0}^{\infty} \frac{\lambda^{* n} \lambda^{m}}{\sqrt{n ! m !}}|n\rangle\langle m| \\
& =\sum_{n, m=0}^{\infty} \frac{|n\rangle\langle m|}{\sqrt{n ! m !}} \int_{0}^{\infty} d r e^{-r^{2}} r^{n+m+1} \int_{0}^{2 \pi} d \theta e^{-i(n-m) \theta}
\end{aligned}
$$

onde a integral em $\theta$ será nula exceto quando $n=m$. Logo,

$$
\frac{1}{\pi} \int|\lambda\rangle\langle\lambda| d^{2} \lambda=2 \sum_{n=0}^{\infty} \frac{|n\rangle\langle n|}{n !} \int_{0}^{\infty} d r e^{-r^{2}} r^{2 n+1} .
$$


Integrando por partes, percebe-se, por indução finita, que após $n$ integrações, finalmente tem-se

$$
\frac{1}{\pi} \int|\lambda\rangle\left\langle\lambda\left|d^{2} \lambda=2 \sum_{n=0}^{\infty} \frac{|n\rangle\langle n|}{n !} \frac{n !}{2}=\sum_{n=0}^{\infty}\right| n\right\rangle\langle n|=1
$$

onde a última soma é a completeza da base de estados $n$.

Os estados coerentes são, no entanto, não-ortogonais:

$$
\begin{aligned}
\left\langle\lambda \mid \lambda^{\prime}\right\rangle & =\sum_{n=0}^{\infty} \frac{\lambda^{* n}}{\sqrt{n !}} e^{-|\lambda|^{2} / 2}\left\langle n\left|\sum_{m=0}^{\infty} \frac{\lambda^{\prime m}}{\sqrt{m !}} e^{-\left|\lambda^{\prime}\right|^{2} / 2}\right| m\right\rangle \\
& =\sum_{n, m=0}^{\infty} \frac{\lambda^{* n} \lambda^{\prime m}}{\sqrt{n !} \sqrt{m !}} e^{-|\lambda|^{2} / 2} e^{-\left|\lambda^{\prime}\right|^{2} / 2}\langle n \mid m\rangle .
\end{aligned}
$$

Como a base de estados $|n\rangle$ é ortogonal, $\langle n \mid m\rangle=\delta_{n, m}$. Desta forma, a Eq. (5.19) só é diferente de zero para $m=n$, portanto

$$
\left\langle\lambda \mid \lambda^{\prime}\right\rangle=e^{\lambda^{*} \lambda^{\prime}} e^{-\frac{\left(|\lambda|^{2}+\left|\lambda^{\prime}\right|^{2}\right)}{2}},
$$

Devido a suas propriedades no que se refere à forma, relação de incerteza, completeza e notável não-ortogonalidade, estes estados possuem uma vasta gama de aplicações em áreas tais como óptica quântica, física matemática [51, 52] e gravitação quântica [53].

\subsection{Aplicação no modelo SIS}

Nesta seção, a ideia é usar a formulação matemática descrita na seção 4.2 e introduzir os estados coerentes como uma nova forma de lidar com o gerador de translações temporais encontrado para o modelo SIS, o qual é não-hermitiano. Seguindo exemplos encontrados em física da matéria condensada e óptica quântica [54], vamos buscar escrever o gerador de translações temporais em função dos operadores bosônicos. Para tanto, tratamos o modelo SIS como um processo bosônico de criação (infecção) e aniquilação (recuperação) e usamos a transformação HP.

De acordo com o Apêndice 1, a transformação HP permite escrever os operadores de spin $\hat{S}^{+}$e $\hat{S}^{-}$em função de $\hat{n}$, $\hat{a}$ e $\hat{a}^{\dagger}$ :

$$
\begin{aligned}
& \hat{S}^{+}=\hat{a}^{\dagger} \sqrt{N-\hat{n}}, \\
& \hat{S}^{-}=\sqrt{N-\hat{n}} \hat{a} .
\end{aligned}
$$


No limite em que a densidade de infectados é baixa $(\hat{n} / N \ll 1$, ou seja, $N \gg 1)$, faz-se uma expansão em série de Taylor até primeira ordem das Eqs. (5.21) e (5.22):

$$
\begin{aligned}
& \hat{S}^{+}=\hat{a}^{\dagger} \sqrt{N}\left(1-\frac{\hat{n}}{2 N}\right), \\
& \hat{S}^{-}=\sqrt{N}\left(1-\frac{\hat{n}}{2 N}\right) \hat{a} .
\end{aligned}
$$

As Eqs. (5.23) e (5.24), quando inseridas na Eq. (4.23), nos fornecem

$\mathcal{H}=-\frac{\alpha}{\sqrt{N}}\left[\frac{\left(\hat{a}^{\dagger}\right)^{2} \hat{a}^{2}}{\sqrt{N}}+\left(\hat{a}^{\dagger}\right)^{2} \hat{a}-\frac{\left(\hat{a}^{\dagger}\right)^{2} \hat{a}}{2 N}-\frac{\left(\hat{a}^{\dagger}\right)^{3} \hat{a}^{2}}{2 N}\right]-\gamma \sqrt{N}\left(\hat{a}-\frac{\hat{a}^{\dagger} \hat{a}^{2}}{2 N}\right)+\left(\gamma+\alpha-\frac{\alpha}{N}\right) \hat{a}^{\dagger} \hat{a}$.

Com isso, temos o gerador de translações temporais em função dos operadores bosônicos e, desta forma, ele pode agir sobre a base dos estados coerentes.

É importante salientar que, no Apêndice 2, é feita a expansão do operador $\hat{S}^{+}$para ordens maiores de $\hat{n}$.

Agora, queremos mostrar como é realizada a expansão dos operadores $\hat{n}, \hat{a}$ e $\hat{a}^{\dagger}$ em função dos estados coerentes e quais resultados podem ser obtidos para as equações dos valores médios desses operadores. Estas equações são importantes para calcular variações temporais das médias, uma vez que a variação temporal da média de $\hat{n}$ representa a variação no tempo do número médio de indíviduos infectados. Já as equações para as variações das médias dos operadores bosônicos representam as variações do surgimento e da extinção de novos casos da doença, que podem permitir ajustar valores para as taxas de infecção $(\alpha)$ e de cura $(\gamma)$.

De forma geral, o valor médio de operador pode ser calculado pelos valores que ele assume em cada configuração vezes a probabilidade de tal configuração ocorrer, a saber:

$$
\langle\hat{B}\rangle=\sum_{\mu} B_{\mu} P_{\mu}
$$

Porém, $P_{\mu}$ é a projecão do vetor probabilidade $|P(t)\rangle$ na configuração $|\mu\rangle$, de modo que a Eq. (5.26) pode ser escrita como

$$
\langle\hat{B}\rangle=\sum_{\mu}\langle\mu|\hat{B}| P(t)\rangle .
$$

Na seção anterior, as propriedades dos estados coerentes foram definidas, de modo que agora, ao introduzir esses estados na formulação matemática que vem 
sendo desenvolvida até aqui, tais propriedades serão utilizadas para calcular as médias e suas evoluções temporais. Começando pelo operador $\hat{a}^{l}$ que diminui o número de infectados numa configuração, considere sua média que, conforme a Eq. (5.27) é dada por:

$$
\left\langle\hat{a}^{l}\right\rangle=\left\langle\mu\left|\hat{a}^{l}\right| P(t)\right\rangle .
$$

Inserindo a completeza dos estados coerentes (Eq. 5.18) na equação anterior, tem-se $\left\langle\hat{a}^{l}\right\rangle=\sum_{\mu}\left\langle\mu\left|\int \frac{d^{2} \lambda^{\prime}}{\pi}\right| \lambda^{\prime}\right\rangle\left\langle\lambda^{\prime}\left|\hat{a}^{l} \int \frac{d^{2} \lambda}{\pi}\right| \lambda\right\rangle\langle\lambda \mid P(t)\rangle=\int \frac{d^{2} \lambda d^{2} \lambda}{\pi^{2}} \sum_{\mu}\left\langle\mu \mid \lambda^{\prime}\right\rangle\left\langle\lambda^{\prime}\left|\hat{a}^{l}\right| \lambda\right\rangle\langle\lambda \mid P(t)\rangle$.

Antes de prosseguir, é importante considerar as seguintes definições:

$$
P\left(\lambda^{*}, t\right)=\langle\lambda \mid P(t)\rangle
$$

que é a mudança de base dos vetores probabilidade para a base dos estados coerentes, e

$$
\rho(\lambda)=\sum_{\mu}\langle\mu \mid \lambda\rangle
$$

que definine-se como a densidade de estados $|\lambda\rangle$ nas configurações $|\mu\rangle$. Verifica-se (ver Apêndice X) que as Eqs. (5.30) e (5.31) são dadas por

$$
\begin{aligned}
P\left(\lambda^{*}, t\right) & =P(t) \sum_{n=0}^{\infty} \frac{\left(\lambda^{*}\right)^{n}}{\sqrt{n}} e^{-|\lambda|^{2} / 2}, \\
\rho(\lambda) & =\frac{e^{-|\lambda|^{2} / 2}}{\pi} \sum_{n=0}^{N} \frac{\lambda^{n}}{\sqrt{n !}}\left(\begin{array}{l}
N \\
n
\end{array}\right)^{1 / 2} .
\end{aligned}
$$

Inserindo essas definições na Eq. (5.29), tem-se:

$$
\left\langle\hat{a}^{l}\right\rangle=\int \frac{d^{2} \lambda d^{2} \lambda}{\pi^{2}} \rho\left(\lambda^{\prime}\right)\left\langle\lambda^{\prime}\left|\hat{a}^{l}\right| \lambda\right\rangle P\left(\lambda^{*}, t\right) .
$$

Pode-se notar que o operador $\hat{a}^{l}$ aparece conectando dois estados coerentes diferentes. Sua ação pode ser calculada, uma vez que sabe-se como o operador de aniquilação age sobre os estados coerentes, de modo que o penúltimo termo do lado direito da Eq. (5.34) fica:

$$
\left\langle\lambda^{\prime}\left|\hat{a}^{l}\right| \lambda\right\rangle=\lambda^{l}\left\langle\lambda^{\prime} \mid \lambda\right\rangle
$$

Assim, pode-se escrever

$$
\left\langle\hat{a}^{l}\right\rangle=\int \frac{d^{2} \lambda}{\pi} P\left(\lambda^{*}, t\right) \lambda^{l} \int \frac{d^{2} \lambda^{\prime}}{\pi} \rho\left(\lambda^{\prime}\right)\left\langle\lambda^{\prime} \mid \lambda\right\rangle .
$$


Agora, convém notar que a integral em $\lambda^{\prime}$ da Eq. (5.36) pode ser calculada. Observe que, dada a definição de $\rho(\lambda)$ - Eq. (5.31), ao aplicar a ela a completeza dos estados coerentes, tem-se

$$
\rho(\lambda)=\sum_{\mu}\langle\mu \mid \lambda\rangle=\int \frac{d^{2} \lambda^{\prime}}{\pi} \rho\left(\lambda^{\prime}\right)\left\langle\lambda^{\prime} \mid \lambda\right\rangle,
$$

sendo este termo do lado direito da Eq. (5.37) exatamente a integral que queremos calcular. Por conseguinte, a Eq. (5.36) pode ser finalmente escrita como

$$
\left\langle\hat{a}^{l}\right\rangle=\int \frac{d^{2} \lambda}{\pi} P\left(\lambda^{*}, t\right) \lambda^{l} \rho(\lambda) .
$$

Também gostaria-se de calcular a média dos operadores $\left(\hat{a}^{\dagger}\right)^{l}$, que age aumentando o número de infectados em uma configuração, e $\hat{n}^{l}$, cujos autovalores fornecem o número de infectados. Um procedimento semelhante ao utilizado para $\hat{a}^{l}$ será aplicado. Tem-se

$$
\left\langle\left(\hat{a}^{\dagger}\right)^{l}\right\rangle=\left\langle\mu\left|\left(\hat{a}^{\dagger}\right)^{l}\right| P(t)\right\rangle=\int \frac{d^{2} \lambda}{\pi} P\left(\lambda^{*}, t\right) \int \frac{d^{2} \lambda^{\prime}}{\pi}\left(\lambda^{\prime *}\right)^{l} \rho\left(\lambda^{\prime}\right)\left\langle\lambda^{\prime} \mid \lambda\right\rangle
$$

onde utiliza-se a relação

$$
\left\langle\lambda^{\prime}\left|\left(\hat{a}^{\dagger}\right)^{l}\right| \lambda\right\rangle=\left(\lambda^{\prime *}\right)^{l}\left\langle\lambda^{\prime} \mid \lambda\right\rangle
$$

uma vez que está sendo calculada a ação do complexo conjugado do operador $\hat{a}^{l}$ sobre o complexo conjugado (bra) de um estado coerente.

Pode-se abrir a última integral da Eq. (5.39) usando a relação de nãoortogonalidade da Eq. (5.20), o que resulta em

$$
\left\langle\left(\hat{a}^{\dagger}\right)^{l}\right\rangle=\int \frac{d^{2} \lambda}{\pi} P\left(\lambda^{*}, t\right) \int \frac{d^{2} \lambda^{\prime}}{\pi}\left(\lambda^{\prime *}\right)^{l} \rho\left(\lambda^{\prime}\right) e^{-\frac{|\lambda|^{2}+\left|\lambda^{\prime}\right|^{2}}{2}} e^{\lambda \lambda^{\prime *}} .
$$

Neste ponto, é importante observar que $\left(\lambda^{\prime *}\right)^{l} e^{\lambda \lambda^{\prime *}}=\left(\frac{\partial}{\partial \lambda}\right)^{l} e^{\lambda \lambda^{\prime *}}$. Deste modo, tem-se:

$$
\begin{aligned}
\left\langle\left(\hat{a}^{\dagger}\right)^{l}\right\rangle & =\int \frac{d^{2} \lambda}{\pi} P\left(\lambda^{*}, t\right)\left(\frac{\partial}{\partial \lambda}\right)^{l} \int \frac{d^{2} \lambda^{\prime}}{\pi} \rho\left(\lambda^{\prime}\right) e^{-\frac{|\lambda|^{2}+\left|\lambda^{\prime}\right|^{2}}{2}} e^{\lambda \lambda^{\prime *}} \\
& =\int \frac{d^{2} \lambda}{\pi} P\left(\lambda^{*}, t\right)\left(\frac{\partial}{\partial \lambda}\right)^{l} \rho(\lambda) .
\end{aligned}
$$

Para o operador $\hat{n}^{l}$, procede-se de maneira semelhante, usando a equação obtida no Apêndice 2:

$$
\hat{n}^{l}=\sum_{m=0}^{l}\left\{\begin{array}{c}
l \\
m
\end{array}\right\}\left(\hat{a}^{\dagger}\right)^{m} \hat{a}^{m}
$$


onde $\sum_{m=0}^{l}\left\{\begin{array}{l}l \\ m\end{array}\right\}$ são os números de Stirling do segundo tipo [55]. Usando essa relação, tem-se:

$$
\left\langle\hat{n}^{l}\right\rangle=\left\langle\mu\left|\hat{n}^{l}\right| P(t)\right\rangle=\int \frac{d^{2} \lambda}{\pi} \int \frac{d^{2} \lambda^{\prime}}{\pi} P\left(\lambda^{*}, t\right) \rho\left(\lambda^{\prime}\right)\left\langle\lambda^{\prime}\left|\sum_{m=0}^{l}\left\{\begin{array}{c}
l \\
m
\end{array}\right\}\left(\hat{a}^{\dagger}\right)^{m} \hat{a}^{m}\right| \lambda\right\rangle .
$$

Sendo

$$
\left\langle\lambda^{\prime}\left|\sum_{m=0}^{l}\left\{\begin{array}{c}
l \\
m
\end{array}\right\}\left(\hat{a}^{\dagger}\right)^{m} \hat{a}^{m}\right| \lambda\right\rangle=\sum_{m=0}^{l}\left\{\begin{array}{c}
l \\
m
\end{array}\right\} \lambda^{m}\left(\lambda^{\prime *}\right)^{m}\left\langle\lambda^{\prime} \mid \lambda\right\rangle,
$$

a Eq. (5.44) fica

$$
\begin{aligned}
\left\langle\hat{n}^{l}\right\rangle & =\int \frac{d^{2} \lambda}{\pi} P\left(\lambda^{*}, t\right) \int \frac{d^{2} \lambda^{\prime}}{\pi} \sum_{m=0}^{l}\left\{\begin{array}{c}
l \\
m
\end{array}\right\} \lambda^{m}\left(\lambda^{\prime *}\right)^{m} \rho\left(\lambda^{\prime}\right)\left\langle\lambda^{\prime} \mid \lambda\right\rangle \\
& =\sum_{m=0}^{l}\left\{\begin{array}{c}
l \\
m
\end{array}\right\} \int \frac{d^{2} \lambda}{\pi} P\left(\lambda^{*}, t\right) \lambda^{m} \int \frac{d^{2} \lambda^{\prime}}{\pi}\left(\lambda^{\prime *}\right)^{m} \rho\left(\lambda^{\prime}\right)\left\langle\lambda^{\prime} \mid \lambda\right\rangle \\
& =\sum_{m=0}^{l}\left\{\begin{array}{c}
l \\
m
\end{array}\right\} \int \frac{d^{2} \lambda}{\pi} P\left(\lambda^{*}, t\right) \lambda^{m}\left(\frac{\partial}{\partial \lambda}\right)^{m} \rho(\lambda),
\end{aligned}
$$

onde foi usada a relação $\left(\lambda^{\prime *}\right)^{m} e^{\lambda \lambda^{\prime *}}=\left(\frac{\partial}{\partial \lambda}\right)^{m} e^{\lambda \lambda^{\prime *}}$.

Sumarizando os resultados obtidos para as médias dos operadores:

$$
\begin{aligned}
\left\langle\hat{a}^{l}\right\rangle & =\int \frac{d^{2} \lambda}{\pi} P\left(\lambda^{*}, t\right) \lambda^{l} \rho(\lambda), \\
\left\langle\left(\hat{a}^{\dagger}\right)^{l}\right\rangle & =\int \frac{d^{2} \lambda}{\pi} P\left(\lambda^{*}, t\right)\left(\frac{\partial}{\partial \lambda}\right)^{l} \rho(\lambda), \\
\left\langle\hat{n}^{l}\right\rangle & =\sum_{m=0}^{l}\left\{\begin{array}{c}
l \\
m
\end{array}\right\} \int \frac{d^{2} \lambda}{\pi} P\left(\lambda^{*}, t\right) \lambda^{m}\left(\frac{\partial}{\partial \lambda}\right)^{m} \rho(\lambda) .
\end{aligned}
$$

Para calcular a evolução temporal dos operadores, deve-se levar em conta que o único termo dependente do tempo nas Eqs. (5.47) é $P\left(\lambda^{*}, t\right)$. Aqui, é importante retornar à Eq. (4.19). De acordo com ela, $d|P(t)\rangle / d t=-\hat{H}|P(t)\rangle$, de modo que pode-se trabalhar com esta equação, inserindo a completeza dos estados coerentes, para reescrevê-la em função do Hamiltoniano do sistema, que já está escrito em função dos operadores de aniquilação e criação, graças à transformação HolsteinPrimakoff. Tem-se, portanto,

$$
\frac{d|P(t)\rangle}{d t}=-\int \frac{d^{2} \lambda^{\prime}}{\pi} \hat{H}\left|\lambda^{\prime}\right\rangle\left\langle\lambda^{\prime} \mid P(t)\right\rangle=-\int \frac{d^{2} \lambda^{\prime}}{\pi} \hat{H}\left|\lambda^{\prime}\right\rangle P\left(\lambda^{\prime *}, t\right)
$$

Multiplicando ambos os lados da eq. (5.48) por $\langle\lambda|$, tem-se

$$
\frac{\partial P\left(\lambda^{*}, t\right)}{\partial t}=-\int \frac{d^{2} \lambda^{\prime}}{\pi}\left\langle\lambda|\hat{H}| \lambda^{\prime}\right\rangle P\left(\lambda^{\prime *}, t\right)=-\int \frac{d^{2} \lambda^{\prime}}{\pi} H\left(\lambda^{*}, \lambda^{\prime}\right)\left\langle\lambda \mid \lambda^{\prime}\right\rangle P\left(\lambda^{\prime *}, t\right),
$$


onde $\left\langle\lambda|\hat{H}| \lambda^{\prime}\right\rangle=H\left(\lambda^{*}, \lambda^{\prime}\right)\left\langle\lambda \mid \lambda^{\prime}\right\rangle$, sendo que $H\left(\lambda^{*}, \lambda^{\prime}\right)$ pode ser calculado graças à Eq. (5.25) e consiste apenas em aplicar os operadores bosônicos aos estados coerentes:

$H\left(\lambda^{*}, \lambda^{\prime}\right)=-\frac{\alpha^{\prime}}{\sqrt{N}}\left[\frac{\lambda^{\prime}}{\sqrt{N}}+1-\frac{1}{2 N}-\frac{\lambda^{*} \lambda^{\prime}}{2 N}\right]\left(\lambda^{*}\right)^{2} \lambda^{\prime}-\gamma^{\prime} \sqrt{N}\left(1+\frac{\lambda^{*} \lambda^{\prime}}{2 N}\right) \lambda^{\prime}+\left(\alpha^{\prime}+\gamma^{\prime}-\frac{\alpha^{\prime}}{N}\right) \lambda^{*} \lambda^{\prime}$.

Portanto, de posse desta equação e considerando que já são conhecidos $H\left(\lambda^{*}, \lambda^{\prime}\right)$ (Eq. (5.50)), $\left\langle\lambda \mid \lambda^{\prime}\right\rangle$ (ortogonalidade) e $P\left(\lambda^{\prime *}, t\right)$ (Eq. (5.32)), pode-se calcular as derivadas temporais das Eqs. (5.47):

$$
\begin{aligned}
\frac{d\left\langle\hat{a}^{l}\right\rangle}{d t} & =\int \frac{d^{2} \lambda}{\pi} \frac{\partial P\left(\lambda^{*}, t\right)}{\partial t} \lambda^{l} \rho(\lambda) \\
& =-\int \frac{d^{2} \lambda d^{2} \lambda^{\prime}}{\pi^{2}} H\left(\lambda^{*}, \lambda^{\prime}\right)\left\langle\lambda \mid \lambda^{\prime}\right\rangle P\left(\lambda^{*}, t\right) \lambda^{l} \rho(\lambda) \\
\frac{d\left\langle\left(\hat{a}^{\dagger}\right)^{l}\right\rangle}{d t} & =\int \frac{d^{2} \lambda}{\pi} \frac{\partial P\left(\lambda^{*}, t\right)}{\partial t}\left(\frac{\partial}{\partial \lambda}\right)^{l} \rho(\lambda) \\
& =-\int \frac{d^{2} \lambda d^{2} \lambda^{\prime}}{\pi^{2}} H\left(\lambda^{*}, \lambda^{\prime}\right)\left\langle\lambda \mid \lambda^{\prime}\right\rangle P\left(\lambda^{*}, t\right)\left(\frac{\partial}{\partial \lambda}\right)^{l} \rho(\lambda), \\
\frac{d\left\langle\hat{n}^{l}\right\rangle}{d t} & =\sum_{m=0}^{l}\left\{\begin{array}{c}
l \\
m
\end{array}\right\} \int \frac{d^{2} \lambda}{\pi} \frac{\partial P\left(\lambda^{*}, t\right)}{\partial t} \lambda^{m}\left(\frac{\partial}{\partial \lambda}\right)^{m} \rho(\lambda) \\
& =-\sum_{m=0}^{l}\left\{\begin{array}{c}
l \\
m
\end{array}\right\} \int \frac{d^{2} \lambda d^{2} \lambda^{\prime}}{\pi^{2}} H\left(\lambda^{*}, \lambda^{\prime}\right)\left\langle\lambda \mid \lambda^{\prime}\right\rangle P\left(\lambda^{*}, t\right) \lambda^{m}\left(\frac{\partial}{\partial \lambda}\right)^{m} \rho(\lambda) .
\end{aligned}
$$

\subsection{Conclusões do capítulo}

Encontradas as equações para as derivadas temporais dos valores médios, pode-se agora relacioná-las aos parâmetros envolvidos no estudo de uma epidemia. Foi feita, neste capítulo, uma analogia entre o processo de transmissão da doença e os níveis de energia de um oscilador harmônico quântico. Inicialmente, temos um número $n$ de infectados, autovalor do operador número $\hat{n}$. A ação do operador $\hat{a}^{\dagger}$ gera uma infecção, o que aumenta o número de infectados, já a ação do operador $\hat{a}$ gera uma cura, o que diminui o número de infectados.

O operador número, ao atuar em uma configuração, tem como autovalor o número total de indivíduos infectados. Desta forma, a variação temporal do valor médio deste operador, $\frac{d\left\langle\hat{n}^{l}\right\rangle}{d t}$, fornece a variação no número médio de indivíduos 
infectados.

O operador destruição, ao atuar em uma configuração, retorna uma nova configuração com um indivíduo infectado a menos. Ao ser aplicado $l$ vezes, diminui de $l$ o número de infectados. Desta forma, a variação temporal de sua média, $\frac{d\left\langle\hat{a}^{l}\right\rangle}{d t}$, representa a variação nos casos de cura e, portanto, é a taxa de cura $\gamma$.

O operador criação, ao atuar em uma configuração, retorna uma nova configuração com um indivíduo infectado a mais. Ao ser aplicado $l$ vezes, aumenta de $l$ o número de infectados. Desta forma, a variação temporal de sua média, $\frac{d\left\langle\left(\hat{a}^{\dagger}\right)^{l}\right\rangle}{d t}$, representa a variação nos casos de infecção e, portanto, é a taxa de infecção $\alpha$.

Com as Eqs. (5.51), pode-se então calcular a evolução temporal do número de infectados e os valores das taxas de infecção e cura, sem a necessidade de considerálas como valores constantes. 


\section{6}

\section{Conclusão e perspectivas}

Processos estocásticos em biologia, tais como epidemias, que envolvem o comportamento coletivo de entidades biológicas, possuem similaridades com sistemas de muitos corpos em física. Nesta dissertação, buscou-se fazer não só uma analogia entre o processo epidêmico SIS e um sistema de spin $1 / 2$, mas também uma equivalência matemática entre eles, e por meio dela chegar a modelos que sejam adequados para os diferentes tipos de limites considerados: populações pequenas e populações grandes. Além disso, os modelos aqui desenvolvidos visam entender e quantificar parâmetros envolvidos em epidemias, como flutuações e taxas de cura e infecção, de modo a melhor prever o comportamento da transmissão dos patógenos.

Neste trabalho, com a formulação matemática desenvolvida no capítulo 4 para o modelo SIS, derivamos uma equação para a densidade de infectados que contém um termo de flutuação $\sigma^{2}(t)$, que surge devido ao caráter estocástico da transmissão de doenças, introduzindo incerteza na densidade de infectados. Verifica-se que $\sigma^{2}(t)$ é um termo que se torna muito relevante para valores de $N$ pequenos (como mostrado na Fig. 4.6). De fato, para os mesmos valores da razão $\gamma / \alpha$, vê-se que o efeito da flutuação é o de aumentar a densidade de infectados no começo do espalhamento da doença e diminuir no equilíbrio, efeito mais pronunciado quanto menor o valor de $N$. Estas simulações mostram, portanto, que para analisar o comportamento das doenças em pequenas populações não se pode desprezar as flutuações na densidade de infectados. Desta forma, a equação compartimental passa a não ser adequada para descrever a dinâmica de transmissão de patógenos em pequenas populações.

Uma vez que uma nova equação compartimental contendo um termo de flu- 
tuações é derivada, torna-se necessário derivar também uma equação para esse novo termo, de modo que seja possível analisar o comportamento das flutuações. A Eq. (4.35) descreve o comportamento ao longo do tempo das flutuações e, juntamente com a Eq. (4.31), formam um sistema fechado do qual pode-se derivar uma equação de segunda ordem para $\langle\rho(t)\rangle$, quando flutuações Gaussianas são consideradas, o que é possível quando $N \gg 1$ e $\gamma / \alpha$ é menor que 0.8. Para flutuações não simétricas, quando $N=10,20$ e $\gamma / \alpha$ tem valores próximos a um, a função de autocorrelação normalizada fornece uma forma de tornar o sistema fechado novamente.

No limite em que a população torna-se muito grande e no qual pode-se considerar que as flutuações são mínimas, o problema torna-se semelhante às ondas de spin com pequenas oscilações, o que nos leva a introduzir a transformação HP. Esta transformação permite trocar os operadores de spin da Eq. (4.23) por operadores bosônicos de criação e aniquilação, tornando possível utilizar os estados coerentes como base. Usando as propriedades destes estados, chega-se a equações que descrevem parâmetros importantes da epidemia, como variação do número de infectados e taxas de infecção e cura.

Como extensão deste trabalho, seria importante fazer a comparação das previsões do modelo com os dados empíricos. Dados epidemiológicos de pequenas comunidades, como por exemplo famílias de nativos que vivem isoladas [56], são uma excelente forma de se verificar os resultados previstos neste trabalho. Também é importante ressaltar que neste trabalho a população foi tratada da maneira mais simples possível, com conexões representadas por um grafo completo, de modo que uma das questões que surge é como o comportamento das flutuações no processo de espalhamento da doença é influenciado pelas incertezas inerentes a grafos com diferentes graus de conectividade.

Além disso, devido ao fato do modelo SIS ser restrito quanto à descrição de doenças, limitando-se aquelas às quais um indivíduo não adquire imunidade após a infecção, outra questão que surge é explorar a descrição de modelos mais complexos, como o SEIRS, no qual tem-se quatro estados diferentes possíveis para cada indivíduo, utilizando a formulação matemática aqui desenvolvida.

Em suma, pode-se dizer que a epidemiologia é uma área em constante desenvolvimento, pois lida com epidemias de doenças conhecidas que surgem a todo 
momento, com doenças novas e também com a evolução dos patógenos, que tornamse cada vez mais resistentes às drogas existentes, de forma que sua evolução deve ser levada em conta na produção de novas vacinas $[57,58]$. Com estas considerações em mente, percebe-se a importância da união de pesquisadores de áreas tais como física, computação, biologia evolutiva e ecologia para adressar os problemas existentes em epidemiologia. 


\section{Apêndice}

\section{Transformação de Holstein-Primakoff}

O Hamiltoniano do processo SIS, na aproximação de campo médio, é dado em termos dos operadores de spin coletivos:

$$
\mathcal{H}=-\frac{\alpha}{N}\left(\hat{n}+\hat{S}^{+}\right) \hat{n}-\gamma \hat{S}^{-}+(\alpha+\gamma) \hat{n}
$$

onde $\hat{n}=\hat{a}^{\dagger} \hat{a}$ é o operador número em função dos operadores bosônicos de levantamento $\left(\hat{a}^{\dagger}\right)$ e abaixamento $(\hat{a})$.

Na transformação de Holstein-primakoff [48, 54, 59], os operadores de spin coletivos são expressados em termos dos operadores bosônicos $\hat{a}$ e $\hat{a}^{\dagger}$ :

$$
\begin{aligned}
& \hat{S}_{z} \equiv \hbar\left(\hat{a}^{\dagger} \hat{a}-N / 2\right), \\
& \hat{S}^{+} \equiv \hbar \hat{a}^{\dagger} \hat{B}^{\dagger}, \\
& \hat{S}^{-} \equiv \hbar \hat{B} \hat{a},
\end{aligned}
$$

onde $N / 2=S$ é o valor máximo de spin, $\hat{B}^{\dagger}$ e $\hat{B}$ são os operadores que devem ser encontrados para realizar a transformação.

As relações de comutação dos operadores de spin são dadas por [60]:

$$
\begin{aligned}
{\left[\hat{S}_{z}, \hat{S}^{+}\right] } & =\hbar \hat{S}^{-} \\
{\left[\hat{S}_{z}, \hat{S}^{-}\right] } & =-\hbar \hat{S}^{+}, \\
{\left[\hat{S}^{+}, \hat{S}^{-}\right] } & =2 \hbar \hat{S}_{z},
\end{aligned}
$$

e serão utilizadas no cálculo dos operadores $\hat{B}^{\dagger}$ e $\hat{B}$.

Começando pela Eq. (1.5), substituindo $\hat{S}_{z}$ e $\hat{S}^{+}$de acordo com as Eqs. (1.2) 
e (1.3), tem-se:

$$
\hbar\left(\hat{a}^{\dagger} \hat{a}-N / 2\right) \hbar \hat{a}^{\dagger} \hat{B}^{\dagger}-\hbar \hat{a}^{\dagger} \hat{B}^{\dagger} \hbar\left(\hat{a}^{\dagger} \hat{a}-N / 2\right)=\hbar^{2} \hat{a}^{\dagger} \hat{B}^{\dagger}
$$

Desenvolvendo a Eq. (1.8) e usando as relações $\hat{n}=\hat{a}^{\dagger} \hat{a}$ e a comutação $\left[\hat{n}, \hat{a}^{\dagger} \hat{B}^{\dagger}\right]=$ $\hat{n} \hat{a}^{\dagger} \hat{B}^{\dagger}-\hat{a}^{\dagger} \hat{B}^{\dagger} \hat{n}$, tem-se:

$$
\left[\hat{n}, \hat{a}^{\dagger} \hat{B}^{\dagger}\right]=\hat{a}^{\dagger} \hat{B}^{\dagger}
$$

Fazendo um procedimento semelhante para a Eq. (1.6), substituindo $\hat{S}_{z}$ e $\hat{S}^{-}$de acordo com as Eqs. (1.2) e (1.4), mas desta vez considerando a relação de comutação $[\hat{n}, \hat{B} \hat{a}]=\hat{n} \hat{B} \hat{a}-\hat{B} \hat{a} \hat{n}$, tem-se:

$$
[\hat{n}, \hat{B} \hat{a}]=-\hat{B} \hat{a}
$$

Para que se possa analisar as Eqs. (1.9) e (1.10), é preciso lembrar da seguinte identidade

$$
[A, B C]=[A, B] C+B[A, C]
$$

onde A, B e C são operadores quaisquer. De posse da identidade da Eq. (1.11), é possível reescrever a Eq. (1.9)

$$
\begin{aligned}
{\left[\hat{n}, \hat{a}^{\dagger} \hat{B}^{\dagger}\right] } & =\left[\hat{n}, \hat{a}^{\dagger}\right] \hat{B}^{\dagger}+\hat{a}^{\dagger}\left[\hat{n}, \hat{B}^{\dagger}\right] \\
\hat{a}^{\dagger} \hat{B}^{\dagger} & =\left[\hat{n}, \hat{a}^{\dagger}\right] \hat{B}^{\dagger}+\hat{a}^{\dagger}\left[\hat{n}, \hat{B}^{\dagger}\right]
\end{aligned}
$$

Aqui, é preciso lembrar da relação de comutação entre $\hat{n}$ e $\hat{a}^{\dagger}$, que fornece $\left[\hat{n}, \hat{a}^{\dagger}\right]=\hat{a}^{\dagger}$, de modo que o lado direito da Eq. (1.12) anula-se com o primeiro termo do lado esquerdo, resultando em:

$$
\left[\hat{n}, \hat{B}^{\dagger}\right]=0
$$

Procedendo de maneira semelhante para a Eq. (1.10), tem-se

$$
\begin{aligned}
{[\hat{n}, \hat{B} \hat{a}] } & =[\hat{n}, \hat{B}] \hat{a}+\hat{B}[\hat{n}, \hat{a}] \\
-\hat{B} \hat{a} & =[\hat{n}, \hat{B}] \hat{a}+\hat{B}[\hat{n}, \hat{a}] .
\end{aligned}
$$

Novamente, usando a relação de comutação $[\hat{n}, \hat{a}]=-\hat{a}$, o termo do lado direito da Eq. (1.14) anula-se com o último termo do lado esquerdo, o que resulta em:

$$
[\hat{n}, \hat{B}]=0
$$


Pelas Eqs. (1.13) e (1.15), pode-se concluir que $\hat{B}^{\dagger}=\hat{B}$, portanto o operador $\hat{B}$ é hermitiano.

A Eq. (1.7) fornece a forma do operador $\hat{B}$. Substituindo os operadores $S^{+}$ e $S^{-}$de acordo com as Eqs. (1.3) e (1.4), tem-se

$$
\hat{a}^{\dagger} \hat{B}^{\dagger} \hat{B} \hat{a}-\hat{B} \hat{a} \hat{a}^{\dagger} \hat{B}^{\dagger}=2\left(\hat{a}^{\dagger} \hat{a}-N / 2\right) .
$$

Aqui, as seguintes relações serão usadas: $\hat{n}=\hat{a}^{\dagger} \hat{a},\left[\hat{a}, \hat{a}^{\dagger}\right]=1 \Rightarrow \hat{a} \hat{a}^{\dagger}=1+\hat{n}$, a comutação da Eq. (1.15), $[\hat{n}, \hat{B}]=0$, e o fato de que $\hat{B}$ é hermitiano. Assim, a Eq. (1.16) fica:

$$
\hat{a}^{\dagger} \hat{B}^{2} \hat{a}-\hat{B}^{2}-\hat{B}^{2} \hat{n}=2 \hat{n}-N
$$

Pode-se observar que, na Eq. (1.17), o primeiro termo contém o operador $\hat{B}$ entre os operadores bosônicos, o que é um empecilho para desenvolver os cálculos, portanto deve-se reescrever este termo de forma a obter uma expressão com a qual se possa trabalhar. A informação disponível é a relação de comutação entre os operadores $\hat{B}$ e $\hat{n}$, portanto procura-se reescrever o primeiro termo da Eq. (1.17) de forma que o operador número apareça. Para tal, escreve-se a relação de comutação entre $\hat{B}^{2}$ e $\hat{a}^{\dagger}$ :

$$
\begin{aligned}
{\left[\hat{a}^{\dagger}, \hat{B}^{2}\right] } & =\hat{a}^{\dagger} \hat{B}^{2}-\hat{B}^{2} \hat{a}^{\dagger} \\
\Rightarrow \hat{a}^{\dagger} \hat{B}^{2} & =\left[\hat{a}^{\dagger}, \hat{B}^{2}\right]+\hat{B}^{2} \hat{a}^{\dagger} .
\end{aligned}
$$

Substituindo a Eq. (1.18) na Eq. (1.17), tem-se

$$
\begin{array}{r}
{\left[\hat{a}^{\dagger}, \hat{B}^{2}\right] \hat{a}+\hat{B}^{2} \hat{a}^{\dagger} \hat{a}-\hat{B}^{2}-\hat{B}^{2} \hat{n}=2 \hat{n}-N} \\
{\left[\hat{a}^{\dagger}, \hat{B}^{2}\right] \hat{a}+\hat{B}^{2} \hat{n}-\hat{B}^{2}-\hat{B}^{2} \hat{n}=2 \hat{n}-N} \\
{\left[\hat{a}^{\dagger}, \hat{B}^{2}\right] \hat{a}-\hat{B}^{2}=2 \hat{n}-N .}
\end{array}
$$

Neste ponto, é necessário fazer a suposição que o operador $\hat{B}^{2}$ pode ser expandido em potências de $\hat{n}$, ou seja,

$$
\hat{B}^{2}=\sum_{k} b_{k} \hat{n}^{k}
$$

e usar esta equação na Eq. (1.19), que fica:

$$
\sum_{k}\left(b_{k}\left[\hat{a}^{\dagger}, \hat{n}^{k}\right] \hat{a}-b_{k} \hat{n}^{k}\right)=2 \hat{n}-N .
$$

Abrindo esta equação para $k=0,1,2,3$ e comparando termo a termo, obtém-se os valores de $b_{k}$. 
- $\mathrm{k}=0$. Fazendo uso da relação de comutação $\left[\hat{a}^{\dagger}, 1\right]=0$, tem-se:

$$
\begin{aligned}
b_{0}\left[\hat{a}^{\dagger}, 1\right] \hat{a}-b_{0} & =2 \hat{n}-N \\
\therefore b_{0} & =N ;
\end{aligned}
$$

- $\mathrm{k}=1$. Fazendo uso das relações de comutação $\left[\hat{a}^{\dagger}, 1\right]=0$ e $\left[\hat{a}^{\dagger}, \hat{n}\right]=-\hat{a}^{\dagger}$, tem-se:

$$
\begin{aligned}
b_{0}\left[\hat{a}^{\dagger}, 1\right]-b_{0}+b_{1}\left[\hat{a}^{\dagger}, \hat{n}\right] \hat{a}-b_{1} \hat{n} & =2 \hat{n}-N \\
\therefore b_{1} & =-1 ;
\end{aligned}
$$

- $\mathrm{k}=2$. Fazendo uso das relações de comutação $\left[\hat{a}^{\dagger}, 1\right]=0,\left[\hat{a}^{\dagger}, \hat{n}\right]=-\hat{a}^{\dagger}$, $\left[\hat{a}^{\dagger}, \hat{n}^{2}\right]=-2 \hat{n} \hat{a}^{\dagger}+\hat{a}^{\dagger}$, tem-se:

$$
\begin{gathered}
b_{0}\left[\hat{a}^{\dagger}, 1\right]+b_{1}\left[\hat{a}^{\dagger}, \hat{n}\right] \hat{a}+b_{2}\left[\hat{a}^{\dagger}, \hat{n}^{2}\right] \hat{a}-b_{0}-b_{1} \hat{n}-b_{2} \hat{n}^{2}=2 \hat{n}-N \\
\therefore b_{2}=0
\end{gathered}
$$

- $\mathrm{k}=3$. Fazendo uso das relações de comutação $\left[\hat{a}^{\dagger}, 1\right]=0,\left[\hat{a}^{\dagger}, \hat{n}\right]=-\hat{a}^{\dagger}$, $\left[\hat{a}^{\dagger}, \hat{n}^{2}\right]=-2 \hat{n} \hat{a}^{\dagger}+\hat{a}^{\dagger}$ e $\left[\hat{a}^{\dagger}, \hat{n}^{3}\right]=-3 \hat{n}^{2} \hat{a}^{\dagger}+2 \hat{n} \hat{a}^{\dagger}-\hat{a}^{\dagger}$, tem-se:

$$
\begin{array}{r}
b_{0}\left[\hat{a}^{\dagger}, 1\right]+b_{1}\left[\hat{a}^{\dagger}, \hat{n}\right] \hat{a}+b_{2}\left[\hat{a}^{\dagger}, \hat{n}^{2}\right] \hat{a}+b_{3}\left[\hat{a}^{\dagger}, \hat{n}^{3}\right] \hat{a}-b_{0}-b_{1} \hat{n}-b_{2} \hat{n}^{2}-b_{3} \hat{n}^{3}=2 \hat{n}-N \\
\therefore b_{3}=0 ; \quad(1.25)
\end{array}
$$

Por indução finita, os termos $b_{k}$ tais que $k \geq 2$ são nulos, de modo que $\hat{B}^{2}$ fica

$$
\begin{aligned}
\hat{B}^{2} & =b_{0}+b_{1} \hat{n}+b_{2} \hat{n}^{2}+b_{3} \hat{n}^{3}=N-\hat{n} \\
\hat{B} & =\sqrt{N-\hat{n}} .
\end{aligned}
$$

Com a Eq. (1.26) em mãos, pode-se finalmente escrever os operadores de spin em termos dos operadores bosônicos e o operador número, substituindo a Eq. nas Eqs. (1.3) e (1.4):

$$
\begin{aligned}
& \hat{S}^{+}=\hbar \hat{a}^{\dagger} \sqrt{N-\hat{n}}, \\
& \hat{S}^{-}=\hbar \sqrt{N-\hat{n}} \hat{a} .
\end{aligned}
$$




\section{Apêndice}

\section{Potências do operador $\hat{n}$}

Ao fazer a transformação HP, encontra-se uma expressão que contém o operador $\hat{n}$ dentro de uma raiz quadrada, de modo que se torna necessário uma expansão da mesma a fim de realizar os cálculos. Na seção 4.2, foi feita uma expansão em série de Taylor até primeira ordem da raiz quadrada para $\hat{n} / \hat{S} \ll 1$. Porém, para acrescentar termos de ordens superiores, é preciso calcular as potências de $\hat{n}$. Considere as seguintes equações:

$$
\begin{aligned}
\hat{n} & =\hat{a}^{\dagger} \hat{a}, \\
\hat{n}^{2} & =\hat{n} \hat{n}=\hat{a}^{\dagger} \hat{a} \hat{n}, \\
\hat{n}^{3} & =\hat{n} \hat{n} \hat{n}=\hat{a}^{\dagger} \hat{a} \hat{n}^{2}, \\
\hat{n}^{4} & =\hat{n} \hat{n} \hat{n} \hat{n}=\hat{a}^{\dagger} \hat{a} \hat{n}^{3} .
\end{aligned}
$$

Ao continuar a desenvolver as potências do operador número conforme feito acima, nota-se que, de modo geral, pode-se escrever

$$
\hat{n}^{l+1}=\hat{a}^{\dagger} \hat{a} \hat{n}^{l} .
$$

Para que seja possível desenvolver as Eqs. (2.30) a (2.32), de modo a chegar a uma expressão geral para $\hat{n}^{l}$, deve-se levar em conta a Eq. (2.33) e a seguinte relação de comutação

$$
\left[\hat{a},\left(\hat{a}^{\dagger}\right)^{l}\right]=l\left(\hat{a}^{\dagger}\right)^{l-1}
$$

pois, no desenvolvimento das potências de $\hat{n}$, termos do tipo $\hat{a}\left(\hat{a}^{\dagger}\right)^{l}$ aparecem, devendo ser substituídos da seguinte maneira:

$$
\hat{a}\left(\hat{a}^{\dagger}\right)^{l}=\left[\hat{a},\left(\hat{a}^{\dagger}\right)^{l}\right]+\left(\hat{a}^{\dagger}\right)^{l} \hat{a} .
$$


Assim sendo, tem-se

$$
\begin{array}{r}
\hat{n}^{2}=\hat{a}^{\dagger} \hat{a} \hat{n}=\hat{a}^{\dagger} \hat{a} \hat{a}^{\dagger} \hat{a}=\hat{a}^{\dagger}\left(1+\hat{a}^{\dagger} \hat{a}\right) \hat{a}=\hat{a}^{\dagger} \hat{a}+\left(\hat{a}^{\dagger}\right)^{2} \hat{a}^{2}, \\
\hat{n}^{3}=\hat{a}^{\dagger} \hat{a} \hat{n}^{2}=\hat{a}^{\dagger} \hat{a}\left[\hat{a}^{\dagger} \hat{a}+\left(\hat{a}^{\dagger}\right)^{2} \hat{a}^{2}\right]=\hat{a}^{\dagger} \hat{a}+3\left(\hat{a}^{\dagger}\right)^{2} \hat{a}^{2}+\left(\hat{a}^{\dagger}\right)^{3} \hat{a}^{3}, \\
\hat{n}^{4}=\hat{a}^{\dagger} \hat{a} \hat{n}^{3}=\hat{a}^{\dagger} \hat{a}\left[\hat{a}^{\dagger} \hat{a}+3\left(\hat{a}^{\dagger}\right)^{2} \hat{a}^{2}+\left(\hat{a}^{\dagger}\right)^{3} \hat{a}^{3}\right]=\hat{a}^{\dagger} \hat{a}+7\left(\hat{a}^{\dagger}\right)^{2} \hat{a}^{2}+6\left(\hat{a}^{\dagger}\right)^{3} \hat{a}^{3}+\left(\hat{a}^{\dagger}\right)^{4} \hat{a}^{4} .
\end{array}
$$

Por indução, concluímos que

$$
\hat{n}^{l}=\sum_{m=0}^{l}\left\{\begin{array}{c}
l \\
m
\end{array}\right\}\left(\hat{a}^{\dagger}\right)^{m} \hat{a}^{m}
$$

onde o termo $\left\{\begin{array}{c}l \\ m\end{array}\right\}$ representa os números de Stirling do segundo tipo, dados pela relação

$$
\left\{\begin{array}{c}
l \\
m
\end{array}\right\}=\frac{1}{m !} \sum_{k=0}^{m}(-1)^{m-k}\left(\begin{array}{c}
m \\
k
\end{array}\right) k^{l} .
$$

De posse da Eq. (2.39), pode-se finalmente escrever a transformação HP completa para $\hat{S}^{+}$:

$$
\begin{aligned}
\frac{\hat{S}^{+}}{\sqrt{N}} & =\hbar \hat{a}^{\dagger}\left[1-\frac{1}{2} \frac{\hat{n}}{N}-\frac{1}{2 !} \frac{1}{4}\left(\frac{\hat{n}}{N}\right)^{2}-\frac{1}{3 !} \frac{3}{8}\left(\frac{\hat{n}}{N}\right)^{3}-\frac{1}{4 !} \frac{15}{16}\left(\frac{\hat{n}}{N}\right)^{4}+\right] \\
& =\hbar \hat{a}^{\dagger}+\hbar \hat{a}^{\dagger} \sum_{l=1}^{\infty} \sum_{m=0}^{l}\left\{\begin{array}{c}
l \\
m
\end{array}\right\} \frac{\left(\hat{a}^{\dagger}\right)^{m} \hat{a}^{m}}{l ! N^{l}} \prod_{k=1}^{l}\left[\frac{2 k-3}{2}\right]
\end{aligned}
$$




\section{Referências $^{1}$}

[1] "The top 10 causes of death". World Health Organization, 2016. 1

[2] G. Chowell, L. Sattenspiel, S. Bansal e C. Viboud, "Mathematical models to characterize early epidemic growth: A review", Physics of Life Reviews, vol. 18, pp. $66-97,2016.1$

[3] H. Heesterbeek, R. M. Anderson, V. Andreasen, S. Bansal, D. De Angelis, C. Dye, K. T. D. Eames, W. J. Edmunds, S. D. W. Frost, S. Funk, T. D. Hollingsworth, T. House, V. Isham, P. Klepac, J. Lessler, J. O. Lloyd-Smith, C. J. E. Metcalf, D. Mollison, L. Pellis, J. R. C. Pulliam, M. G. Roberts, C. Viboud e, "Modeling infectious disease dynamics in the complex landscape of global health", Science, vol. 347, no. 6227, 2015.

[4] R. Pastor-Satorras e A. Vespignani, "Epidemic spreading in scale-free networks", Phys. Rev. Lett., vol. 86, pp. 3200-3203, Apr 2001. 1

[5] R. Folman, P. Krüger, D. Cassettari, B. Hessmo, T. Maier e J. Schmiedmayer, "Controlling cold atoms using nanofabricated surfaces: Atom chips", Phys. Rev. Lett., vol. 84, pp. 4749-4752, May 2000. 1

[6] W. Ketterle, K. B. Davis, M. A. Joffe, A. Martin e D. E. Pritchard, "High densities of cold atoms in a dark spontaneous-force optical trap", Phys. Rev. Lett., vol. 70, pp. 2253-2256, Apr 1993. 1

[7] B.-P. E. Dawson Peter M., Werkman Marleen e T. M. J., "Epidemic predictions in an imperfect world: modelling disease spread with partial data", Proceedings of the Royal Society B: Biological Sciences, vol. 282. 1

\footnotetext{
${ }^{1}$ De acordo com o estilo LMSC (baseado no estilo IEEE Transactions - Electrical and Electronics Engineers).
} 
[8] R. Ross, Memoirs, with a Full Account of the Great Malaria Problem and Its Solution. Creative Media Partners, LLC, 2015. 1

[9] J. C. Blackwood e L. M. Childs, "An introduction to compartmental modeling for the budding infectious disease modeler", Letters in Biomathematics, vol. 5, no. 1 , pp. $195-221,2018.2$

[10] J. B. Bassingthwaighte, E. Butterworth, B. Jardine e G. M. Raymond, Compartmental Modeling in the Analysis of Biological Systems. Totowa, NJ: Humana Press, 2012. 2

[11] I. Newton, Philosophice Naturalis Principia Mathematica. Benjamin Motte, 1687. 5

[12] P. M. Sharp e B. H. Hahn, "Origins of hiv and the aids pandemic", Cold Spring Harbor Perspectives in Medicine, vol. 1, no. 1, 2011. 5

[13] W. Bank, "The economic impact of the 2014 ebola epidemic : short and medium term estimates for west africa (english)". World Bank Group, 2014. 6

[14] A. Torres, "Surto de dengue cancela carnaval e cria gabinete de crise em sc". Uol Notícias, 2015. 8

[15] P. Piot e T. Quinn, "Response to the aids pandemic - a global health model", The New England journal of medicine, vol. 368, pp. 2210-8, 062013.8

[16] "What is the pandemic (h1n1) 2009 virus?". World Health Organization, 2010. 8

[17] T. Miles, "Who leadership admits failings over ebola, promises reform". Reuters, 2015. 9

[18] L. Kapiriri e A. Ross, "The politics of disease epidemics: a comparative analysis of the sars, zika, and ebola outbreaks", Global Social Welfare, Sep 2018. 9

[19] P. Farmer, "Social inequalities and emerging infectious diseases", Emerging infectious diseases, vol. 2, pp. 259-269, 7 1996. 9

[20] "Measles". World Health Organization, 2019. 9 
[21] "Une famille de français soupçonnée d'avoir réintroduit la rougeole au costa rica”. Le Monde, 2019. 9

[22] T. A. Cockburn, "Infectious diseases in ancient populations", Current Anthropology, vol. 12 , no. 1 , pp. 45-62, 1971. 11

[23] D. Bernoulli e S. Blower, "An attempt at a new analysis of the mortality caused by smallpox and of the advantages of inoculation to prevent it", Reviews in Medical Virology, vol. 14, no. 5, pp. 275-288, 2004. 11

[24] J. Snow, On the Mode of Communication of Cholera. John Churchill, 1855. 11

[25] M. A. G. Kermack William Ogilvy e W. G. Thomas, "A contribution to the mathematical theory of epidemics", Proceedings of the Royal Society of London, vol. 115 , no. $5,1927.12$

[26] H. Hethcote, "The mathematics of infectious diseases", SIAM Review, vol. 42, no. 4, pp. 599-653, 2000. 12

[27] T. Harko, F. S. N. Lobo e M. K. Mak, "Exact analytical solutions of the Susceptible-Infected-Recovered (SIR) epidemic model and of the SIR model with equal death and birth rates", arXiv e-prints, p. arXiv:1403.2160, Mar 2014.

[28] W. J. Brauer F., van den Driessche P., Compartmental Models in Epidemiology, vol. 1945, ch. 2, pp. 19-79. Springer, Berlin, Heidelberg, 2008. 12

[29] S. D. Valle, H. Hethcote, J. Hyman e C. Castillo-Chavez, "Effects of behavioral changes in a smallpox attack model", Mathematical Biosciences, vol. 195, no. 2, pp. $228-251,2005.13$

[30] L. Kong, J. Wang, W. Han e Z. Cao, "Modeling heterogeneity in direct infectious disease transmission in a compartmental model", International Journal of Environmental Research and Public Health, vol. 13, no. 3, 2016. 14

[31] R. Pastor-Satorras e A. Vespignani, "Epidemic dynamics and endemic states in complex networks", Phys. Rev. E, vol. 63, p. 066117, May 2001. 14

[32] M. D. Shirley e S. P. Rushton, "The impacts of network topology on disease spread", Ecological Complexity, vol. 2, no. 3, pp. 287 - 299, 2005. 
[33] M. L. Bansal S, Grenfell BT, "When individual behaviour matters: homogeneous and network models in epidemiology", Journal of the Royal Society, Interface, vol. 4, p. 879-891, Oct 2007.

[34] R. Pastor-Satorras, C. Castellano, P. Van Mieghem e A. Vespignani, "Epidemic processes in complex networks", Rev. Mod. Phys., vol. 87, pp. 925-979, Aug 2015.

[35] H. Rahmandad e J. Sterman, "Heterogeneity and network structure in the dynamics of diffusion: Comparing agent-based and differential equation models", Manage. Sci., vol. 54, pp. 998-1014, maio 2008. 14

[36] W. Delva, G. E. Leventhal e S. Helleringer, "Connecting the dots: network data and models in hiv epidemiology", AIDS (London, England), vol. 30, p. 2009 -2020, August 2016. 15

[37] N. T. J. Bailey, "Some stochastic models for small epidemics in large populations", Journal of the Royal Statistical Society. Series C (Applied Statistics), vol. 13 , no. 1 , pp. 9-19, 1964. 15

[38] L. F. G. Priscilla E. Greenwood, Stochastic Epidemic Modeling. In: Chowell G., Hyman J.M., Bettencourt L.M.A., Castillo-Chavez C. (eds) Mathematical and Statistical Estimation Approaches in Epidemiology, ch. 2, pp. 31-52. Springer, Dordrecht, 2009. 15

[39] A. L.J.S., An Introduction to Stochastic Epidemic Models., vol. 1945, ch. 3, pp. 19-79. Springer, Berlin, Heidelberg, 2008. 15

[40] S. P. Silal, F. Little, K. I. Barnes e L. J. White, "Sensitivity to model structure: a comparison of compartmental models in epidemiology", Health Systems, vol. 5, no. 3, pp. 178-191, 2016. 16

[41] H. H.W., Three Basic Epidemiological Models. In: Levin S.A., Hallam T.G., Gross L.J. (eds) Applied Mathematical Ecology. Biomathematics, vol. 18, ch. 5, pp. 119-144. Springer, Berlin, Heidelberg, 1989. 18

[42] L. Perez e S. Dragicevic, "An agent-based approach for modeling dynamics of contagious disease spread", International Journal of Health Geographics, vol. 8, p. 50, Aug 2009. 20 
[43] M. Azimi, Y. Jamali e M. R. K. Mofrad, "Accounting for diffusion in agent based models of reaction-diffusion systems with application to cytoskeletal diffusion", PLOS ONE, vol. 6, pp. 1-11, 09 2011. 20

[44] G. M. Nakamura, A. C. P. Monteiro, G. C. Cardoso e A. S. Martinez, "Efficient method for comprehensive computation of agent-level epidemic dissemination in networks", Scientific Reports, vol. 7, p. 40885, 1 2017. 21

[45] G. Nakamura, N. Gomes, G. Cardoso e A. Martinez, "Robust parameter determination in epidemic models with analytical descriptions of uncertainties", arXiv e-prints, p. arXiv:1807.05301, Jul 2018. 21

[46] I. Oppenheim e K. E. Shuler, "Master equations and markov processes", Phys. Rev., vol. 138, pp. B1007-B1011, May 1965. 26

[47] R. Baxter, Exactly Solved Models in Statistical Mechanics. Dover books on physics, Dover Publications, 2007. 43

[48] T. Holstein e H. Primakoff, "Field dependence of the intrinsic domain magnetization of a ferromagnet", Phys. Rev., vol. 58, pp. 1098-1113, Dec 1940. 44, 61

[49] D. Griffiths, Introduction to Quantum Mechanics. Cambridge University Press, 2016. 45

[50] R. J. Glauber, "Coherent and incoherent states of the radiation field", Phys. Rev., vol. 131, pp. 2766-2788, Sep 1963. 47

[51] A. O. Barut e L. Girardello, "New "coherent" states associated with noncompact groups", Comm. Math. Phys., vol. 21, no. 1, pp. 41-55, 1971. 50

[52] A. M. Perelomov, "Coherent states for arbitrary lie group", Comm. Math. Phys., vol. 26 , no. 3 , pp. $222-236,1972.50$

[53] B. Bahr e T. Thiemann, "Gauge-invariant coherent states for loop quantum gravity: II. non-abelian gauge groups", Classical and Quantum Gravity, vol. 26, p. 045012, feb 2009. 50 
[54] E. Ressayre e A. Tallet, "Holstein-primakoff transformation for the study of cooperative emission of radiation", Phys. Rev. A, vol. 11, pp. 981-988, Mar 1975. 50,61

[55] O. P. Ronald L. Graham, Donald E. Knuth Concrete Mathematics, p. 244, 1988. 54

[56] M. Laskowski, L. C. Mostaço-Guidolin, A. L. Greer, J. Wu e S. M. Moghadas, "The impact of demographic variables on disease spread: Influenza in remote communities", Scientific Reports, vol. 1, no. 115, 2011. 58

[57] S. Gandon e T. Day, "The evolutionary epidemiology of vaccination", Journal of the Royal Society, Interface, vol. 4, no. 16, pp. 803-17, 2007. 59

[58] S. Gandon, M. J. Mackinnon, S. Nee e A. F. Read, "Imperfect vaccines and the evolution of pathogen virulence", Nature, vol. 414, pp. 751-756, 2001. 59

[59] S. Salinas, Introdução a Física Estatística Vol. 09. EDUSP, 1997. 61

[60] C. Cohen-Tannoudji, B. Diu e F. Laloe, Quantum Mechanics. No. v. 1 em Quantum Mechanics, Wiley, 1991. 61 